\title{
Resultados sobre álgebras alternativas
}

Carlos Córdova Silva

\author{
Dissertação apresentada \\ ao \\ Instituto de Matemática e Estatística \\ da \\ Universidade de São Paulo \\ para a obtenção do grau de Mestre \\ em \\ Matemática \\ Área de concentração: Álgebra \\ Orientador: Prof. Dr. Roberto Celso Fabrício Costa
}

Durante a elaboração deste trabalho, o autor recebeu apoio financeiro do CNPq

- São Paulo, Setembro de 1997 - 
Este exemplar corresponde à redação final da dissertação devidamente corrigida e defendida por Carlos Córdova Silva e aprovada pela comissão julgadora.

São Paulo, 16 de Outubro.

Banca examinadora:

- Prof. Dr. Roberto Celso Fabrício Costa (Orientador) - IME - USP

- Prof. Dr. César Polcino - IME - USP

- Prof. Dr. Juan Carlos Fernández - Universidad de Oviedo. 
À doce memória de

Rebeca San Martin. 


\section{Agradecimentos}

Aos meus pais, pelo carinho que me demonstram dia a dia e pelo apoio que me têm dado durante todos estes anos, sem o qual, dificilmente teria chegado onde me encontro.

À Marcela, que foi minha fonte de inspiração e que apesar da distância, conseguiu com seu amor, dar um significado novo à minha vida.

Às novas amizades que encontrei, pelos bons momentos que vivemos juntos e pela ajuda que me outorgaram nas situações difíceis, especialmente ao Roberto, à Laura, ao Iván e ao meu melhor amigo brasileiro, Júlio (El Minerito).

Ao Marcelo e ao Hernán, meus amigos de sempre, junto aos quais descobri a importância e beleza da verdadeira amizade.

À Lúcia pela constante alegria que transmite e pela ajuda desinteressada cada vez que eu precisei.

Com muito carinho agradeço também ao meu orientador, Roberto, por tudo que fez por mim, pelos conselhos, pelo apoio durante minha estadia no Brasil e pelo grato que é conhecer uma pessoa como ele, que além de bom matemático e um grande ser humano.

A Deus.

Carlos Córdova Silva. 


\begin{abstract}
We find some identities in the variety of alternative algebras, some elements in the associative and the commutative center of this variety and in the sub-variety generated by the free alternative algebra of rank 3. Also, we prove that for an arbitrary $n \in N$ holds a strict inclusion between the sub-algebra generated by the free alternative algebra of rank $n$ and $2^{n}+1$. In addition, we find a basis of the free alternative algebra of rank 3 and prove that this algebra has no nilpotent elements.
\end{abstract}

\title{
Resumo
}

Encontramos várias identidades na variedade das álgebras alternativas, elementos dos centros associativo e comutativo desta variedade e da sub-variedade gerada pela álgebra alternativa livre de posto 3 . Provamos também que para um $n \in N$ arbitrário existe uma incluso estrita entre as sub-variedades geradas pelas álgebras alternativas de posto $n$ e $2^{n}+1$. Além do mais, encontramos uma base da álgebra alternativa livre de posto $3 \mathrm{e}$ provamos que esta álgebra no têm elementos nilpotentes. 


\section{Sumário}

Introdução 2

1 Preliminares 3

1.1 Variedades e linearizações . . . . . . . . . . . . . . . . 3

1.2 Resultados básicos em álgebras alternativas . . . . . . . . . . 7

1.3 Álgebras de composição . . . . . . . . . . . . . . . . . 9

2 Centros de álgebras alternativas $\quad 12$

2.1 Elementos do centro associativo . . . . . . . . . . . . . . 12

2.2 Elementos do centro comutativo . . . . . . . . . . . . . . 19

2.3 Centros de álgebras alternativas com 3 geradores . . . . . . . . 24

3 O problema de Shirshov $\quad 30$

3.1 Algumas relações em Alt $_{r}$. . . . . . . . . . . . . . 30

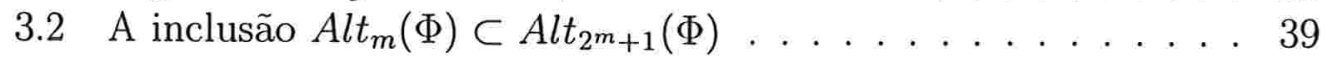

4 Elementos nilpotentes na álgebra alternativa livre com 3 geradores

4.1 Notação e resultados básicos . . . . . . . . . . . . . . . . . 45

4.2 Identidades em álgebras alternativas com 3 geradores . . . . . 48

4.3 Prova do resultado principal . . . . . . . . . . . . 63

$\begin{array}{ll}\text { Bibliografia } & 83\end{array}$ 


\section{Introdução}

O estudo das álgebras alternativas se divide fundamentalmente em duas grandes áreas. Por um lado, o estudo de radicais e elementos nilpotentes de álgebras alternativas livres ([1], [5]). Pelo outro, o estudo dos centros destas álgebras ([2], [4] e [9]).

Nessa dissertação coletamos alguns resultados que têm sido obtidos em ambas as áreas.

No Capítulo 1 introduzimos conceitos básicos assim como alguns resultados obtidos em torno a linearizações parciais. Ainda neste capítulo mostramos resumidamente o processo de Cayley-Dickson para construir álgebras de composição a partir de um corpo arbitrário e damos uma representação matricial à álgebra de Cayley-Dickson.

No capítulo 2 encontramos elementos do centro associativo e do centro comutativo da álgebra alternativa livre. Encontramos também funções $r_{i}(x, y)$, $(i=1,2,3)$ com valores no centro associativo e tais que $r_{1}(x, y) x^{2}+r_{2}(x, y) x+$ $r_{3}(x, y)=0$ para quaisquer $x, y \in A l t[X]$, o que podemos interpretar dizendo que a álgebra alternativa livre é quadrática sobre seu centro associativo. Além do mais, encontramos elementos do centro comutativo e do centro da álgebra alternativa livre de posto 3 .

No Capítulo 3 estudamos a variedade gerada pelo processo de Birkhoff a partir da álgebra alternativa livre com $n$ geradores e respondemos negativamente a pergunta formulada por Shirshov sobre a estabilização da seqüência de sub-variedades $A l t_{1}(\Phi) \subseteq A l t_{2}(\Phi) \subseteq \cdots \subseteq A l t_{n}(\Phi) \subseteq A l t(\Phi)$ se estabiliza.

O quarto Capítulo é dedicado a prova da não existencia de elementos nilpotentes na álgebra alternativa livre de posto $3\left(A_{3}\right)$. Durante a demonstração é construida uma base do ideal gerado pelos associadores nesta álgebra $\left(D\left(A_{3}\right)\right)$. Daqui e observando que $A_{3} / D\left(A_{3}\right) \cong A s s\left[x_{1}, x_{2}, x_{3}\right]$, é fácil construir uma base da própria álgebra $A_{3}$. 


\section{Capítulo 1}

\section{Preliminares}

Neste trabalho são usados vários resultados básicos da teoria das álgebras alternativas. Porisso, e pensando nos leitores que eventualmente não estão familiarizados com o tema, vamos listar as principais definições assim como alguns resultados dentre aqueles que vamos usar nos capítulos posteriores. Estes resultados podem ser encontrados, com detalhes, em [12] e em [6].

Em todo este trabalho, os anéis de escalares são comutativos, associativos com unidade 1 e as álgebras com coeficientes nestes anéis não são necessariamente associativas.

\subsection{Variedades e linearizações}

Dado um conjunto $X=\left\{x_{\alpha}\right\}$, agregamos dois novos símbolos, os parênteses esquerdo e direito, obtendo um novo conjunto que denotamos por $X^{*}=$ $X \cup\{()$,$\} . Consideremos agora o conjunto Y$ de todas as sequências finitas de elementos de $X^{*}$ e definamos indutivamente o conjunto $V[X] \subset Y$ da seguinte maneira: Se $x_{1}, x_{2} \in X$ e $u, v \in V[X] / X$, então os elementos $x_{1} x_{2}$, $x_{1}(u), v\left(x_{2}\right)$ e $(u)(v)$ pertencem a $V[X]$. Além disso, todo elemento de $V[X]$ é obtido desta forma. $V[X]$ é chamado de conjunto de palavras não associativas (ou monômios não associativos) de elementos de $X$. Dado $v \in V[X]$, chamaremos de comprimento de $v$, denotado por $d(v)$, o número de elementos de $X$ que aparecem em $v$. Definimos também o produto de duas palavras não associativas pelas seguintes regras: Se $x_{1}, x_{2} \in X$ e $u, v \in V[X] \backslash X$, então $x_{1} \cdot x_{2}:=x_{1} x_{2}, x_{1} \cdot u:=x_{1}(u), v \cdot x_{2}:=v\left(x_{2}\right)$ e $u \cdot v:=(u)(v)$.

Consideremos agora o $\Phi$-módulo livre $\Phi[X]$ gerado por $V[X]$. Estendendo 
o produto definido em $V[X]$ a $\Phi[X]$ como segue:

$$
\left(\sum \alpha_{i} u_{i}\right)\left(\sum \beta_{j} v_{j}\right)=\sum \alpha_{i} \beta_{j}\left(u_{i} v_{j}\right)
$$

onde $\alpha_{i}, \beta_{j} \in \Phi$ e $u_{i}, v_{j} \in V[X]$, obtemos uma $\Phi$-álgebra $\Phi[X]$ que chamamos de álgebra livre sobre $\Phi$ com conjunto de geradores $X$.

A seguir, introduzimos o conceito de variedade sobre um anel $\Phi$ junto com duas das ferramentas que usaremos mais frequentemente, as identidades de uma variedade e as linearizações parciais delas.

Consideremos um conjunto enumerável de letras $X=\left\{x_{1}, x_{2}, \ldots\right\}$ e $\Phi[X]$ a álgebra livre (não associativa) gerada por $X$. Se $f \in \Phi[X]$, então como em $f$ aparece uma quantidade finita de $x_{i}$ 's podemos supor $f=f\left(x_{1}, \ldots, x_{n}\right)$. Se A é uma $\Phi$-álgebra e se para cada $n$-upla $\left(a_{1}, \ldots, a_{n}\right) \in A^{n}$ temos que $f\left(a_{1}, \ldots, a_{n}\right)=0$, onde $f\left(a_{1}, \ldots, a_{n}\right)$ é o elemento obtido por substituição de $\left(a_{1}, \ldots, a_{n}\right)$ no polinômio não associativo $f\left(x_{1}, \ldots, x_{n}\right)$, então dizemos que $f$ é uma identidade em A. Dizemos também que $f$ é uma identidade na classe $M$ se $f$ for uma identidade em cada álgebra da classe. Assim, por exemplo, $f\left(x_{1}, x_{2}\right)=x_{1}^{2} x_{2}-x_{1}\left(x_{1} x_{2}\right)$ é uma identidade na classe das álgebras alternativas. Denotaremos por $T(M)$ o subconjunto de $\Phi[X]$ formado por todas as identidades da classe $M$. É claro que $T(M)$ é um ideal de $\Phi[X]$.

Inversamente, dado um subconjunto $I \subseteq \Phi[X]$, a classe $M$ de todas as $\Phi$-álgebras satisfazendo cada identidade de $I$ é chamada de variedade de $\Phi$ álgebras definida pelo conjunto de identidades $I$. Por exemplo, a identidade $f\left(x_{1}, x_{2}, x_{3}\right)=\left(x_{1} x_{2}\right) x_{3}-x_{1}\left(x_{2} x_{3}\right)$ define a variedade das álgebras associativas, $g\left(x_{1}, x_{2}\right)=x_{1} x_{2}-x_{2} x_{1}$ define a variedade das álgebras comutativas, $\mathrm{o}$ conjunto de identidades $I=\left\{f\left(x_{1}\right)=x_{1}^{2}, g\left(x_{1}, x_{2}, x_{3}\right)=\left(x_{1} x_{2}\right) x_{3}+\left(x_{2} x_{3}\right) x_{1}+\right.$ $\left.\left(x_{3} x_{1}\right) x_{2}\right\}$ define a variedade das álgebras de Lie, etc.

Dada uma variedade $M$ de $\Phi$-álgebras e $F$ uma álgebra de $M$ com conjunto de geradores $X$, diremos que $F$ é livre na variedade $M$ com conjunto de geradores $X$, se para toda álgebra $A$ de $M$ e toda aplicação $\sigma: X \longrightarrow A, \sigma$ pode ser estendido de maneira única a um homomorfismo $\bar{\sigma}: F \longrightarrow A$.

Um resultado conhecido sobre variedades de álgebras é devido a Birkhoff. Este diz que uma classe $K$, não vazia, de álgebras sobre um anel (comutativo) com unidade, é uma variedade se e somente se $K$ é fechada sob tomar subálgebras, imagens homomorfas e produtos diretos. Desta forma, vemos que existem dois caminhos para construir uma variedade $M$ sobre um anel $\Phi$. A primeira, como já vimos, é tomando todas as $\Phi$-álgebras satisfazendo 
cada identidade de um conjunto $I \subset \Phi[X]$. A segunda é tomar $\bar{I}$ sendo o ideal gerado por $I$ em $\Phi[X]$ e considerar a $\Phi$-álgebra livre $\Phi[X] / \bar{I}$ na variedade $M$. Logo, a partir de $\Phi[X] / \bar{I}$, tomando subálgebras, imagens homomorfas e produtos diretos até obter uma classe fechada sob este processo (que chamaremos processo de Birkhoff), obtemos a variedade em questão. Denotaremos por $\Phi_{M}[X]$ a álgebra livre na variedade $M$. Para as álgebras alternativa e associativa livres usaremos também as notações $A l t[X]$ e $A s s[X]$, respectivamente.

Diremos que um polinômio não associativo $f \in \Phi[X]$ é homogêneo se é soma de monômios, cada um dos quais tem o mesmo grau em cada variável. É claro que dado $f \in \Phi[X]$ podemos escrever $f=f_{1}+\ldots+f_{r}$ onde cada $f_{i}$ é homogêneo e $r$ é minimal. Tais $f_{i}$ são chamados componentes homogêneas de $f$.

Uma variedade $M$ é dita homogênea se, para cada $f \in T(M)$, todas as componentes homogêneas de $f$ também pertencem a $T(M)$. Sabe-se que as variedades mais significativas são homogêneas.

Agora, com esta notação, temos que toda variedade definida por um conjunto de identidades homogêneas de grau $\leq 2$ em cada variável é homogênea, ver ([12, p.13]). Em particular, a variedade das álgebras associativas, das álgebras de Lie e das álgebras alternativas são homogêneas.

Vamos descrever, a seguir, o processo de linearização de identidades já que em muitos casos, cada linearização de uma identidade em uma variedade $M$ é também uma identidade em $M$.

Dado $f \in \Phi[X]$ e tomando $y \notin X$, definamos a seguinte família $\Delta(y)=$ $\left\{\Delta_{i}^{k}(y): i=1,2, \ldots, k=1,2, \ldots\right\}$ de transformações lineares do $\Phi$ - módulo $\Phi[X]$, satisfazendo:

i) $\Delta_{i}^{0}(y)=I d$ (transformação identidade)

ii) $x_{s} \Delta_{i}^{k}(y)=0$ se $k>1$ ou $i \neq s$

iii) $x_{i} \Delta_{i}^{1}(y)=y$

iv) $(u v) \Delta_{i}^{k}(y)=\sum_{r+s=k}\left(u \Delta_{i}^{r}(y)\right)\left(v \Delta_{i}^{s}(y)\right)$

onde $u$ e $v$ são monômios de $\Phi[X]$ e $x_{j} \in X$. Notemos que os operadores lineares $\Delta_{i}^{k}$ estão bem definidos a partir de i),.., iv). Tais operadores $\Delta_{i}^{k}$ são chamados linearizações parciais e se $f \in \Phi[X], f \Delta_{i}^{k}(y)$ é uma linearização parcial do polinômio $f$.

Para entender melhor como operam ditas transformações temos o seguinte 
resultado (ver $[12$, p.9]).

Proposição 1 Se $u=u\left(x_{1}, \ldots, x_{n}\right)$ é um monômio de $\Phi[X]$ de grau $m$ em $x_{i}$ então, para todo $k \leq m$

$$
u \Delta_{i}^{k}(y)=u_{1}+\ldots+u_{\left(\begin{array}{c}
m \\
k
\end{array}\right)}
$$

onde $u_{1}, \ldots, u_{\left(\begin{array}{c}m \\ k\end{array}\right)}$ são todos os monômios obtidos ao substituir $k$ ocorrências de $x_{i}$ no monômio $u$ por $y$, em todas as ordens possíveis.

Por exemplo, $\left(x_{1}^{2}\left(x_{2} x_{1}\right)\right) \Delta_{1}^{2}(y)=y^{2}\left(x_{2} x_{1}\right)+y\left(x_{1}\left(x_{2} y\right)\right)+x_{1}\left(y\left(x_{2} y\right)\right)$.

A seguir, provaremos um resultado simples, porém útil, em relação às linearizações parciais.

Lema 1 Se $v=v\left(x_{1}, \ldots, x_{n}\right)$ é um monômio multilinear em $\Phi[X]$, então para todo $f_{1}, \ldots, f_{n} \in \Phi[X]$, temos a igualdade

$$
v\left(f_{1}, \ldots, f_{n}\right) \Delta_{i}^{k}(y)=\sum_{k_{1}+\ldots+k_{n}=k} v\left(f_{1} \Delta_{i}^{k_{1}}(y), \ldots, f_{n} \Delta_{i}^{k_{n}}(y)\right)
$$

onde $v\left(f_{1}, \ldots, f_{n}\right)$ é o polinômio não associativo obtido ao substituir as variáveis $x_{i}$ pelos polinômios não associativos $f_{i}$.

Demonstração: Denotemos $v(f)=v\left(f_{1}, \ldots, f_{n}\right)$ e $\Delta_{i}^{k}(y)=\Delta_{i}^{k}$. Apliquemos indução sobre $n$. Se $n=1$ o resultado é claro. Suponhamos que o resultado é verdadeiro para $n<n_{0}$ e tomemos $v$ com $d(v)=n_{0}$. Então, podemos escrever $v=v_{1} v_{2} \operatorname{com} d\left(v_{1}\right)=m<n_{0}$ e $d\left(v_{2}\right)=l<n_{0}$ e, pela própria definição das linearizações, temos que

$$
\begin{aligned}
v(f) \Delta_{i}^{k}= & \left(v_{1}(f) v_{2}(f)\right) \Delta_{i}^{k}(y)=\sum_{r+s=k} v_{1}\left(f \Delta_{i}^{r}\right) v_{2}\left(f \Delta_{i}^{s}\right) \\
= & \sum_{r+s=k}\left\{\left(\sum_{r_{1}+\ldots+r_{m}=r} v_{1}\left(f_{1} \Delta_{i}^{r_{1}}(y), \ldots, f_{m} \Delta_{i}^{r_{m}}(y)\right)\right) \times\right. \\
& \left.\quad\left(\sum_{s_{1}+\ldots+s_{l}=s} v_{2}\left(f_{m+1} \Delta_{i}^{s_{1}}(y), \ldots, f_{n_{0}} \Delta_{i}^{s_{l}}(y)\right)\right)\right\} \\
= & \sum_{k_{1}+\ldots+k_{n_{0}}=k} v_{1}\left(f_{1} \Delta_{i}^{k_{1}}, \ldots, f_{m} \Delta_{i}^{k_{m}}\right) v_{2}\left(f_{m+1} \Delta_{i}^{k_{m+1}}, \ldots, f_{n_{o}} \Delta_{i}^{k_{n_{0}}}\right) \\
= & \sum_{k_{1}+\ldots+k_{n_{0}}=k} v\left(f_{1} \Delta_{i}^{k_{1}}, \ldots, f_{n_{o}} \Delta_{i}^{k_{n_{0}}}\right)
\end{aligned}
$$

Portanto o Lema 1 fica provado. 


\subsection{Resultados básicos em álgebras alternati- vas}

Comecemos, como é natural, definindo nosso objeto de estudo.

Definição 1 Uma álgebra A sobre o anel $\Phi$ é dita alternativa se para quaisquer $x, y \in A$ são satisfeitas as seguintes equações, chamadas de leis alternativas direita e esquerda respectivamente:

$$
(x y) y=x y^{2} \quad x(x y)=x^{2} y
$$

Definição 2 Uma álgebra A sobre o anel $\Phi$ é dita de potências associativas se um elemento qualquer de A gera uma subálgebra associativa.

Notação 1 Numa álgebra A sobre $\Phi$ chamamos de associador dos elementos $x, y, z \in A$ o elemento

$$
(x, y, z):=(x y) z-x(y z)
$$

e de comutador de $x, y$ o elemento

$$
[x, y]:=x y-y x
$$

Se $\frac{1}{2} \in \Phi$ também definimos o produto de Jordan de $x$ e $y$ em $A$ por

$$
x \circ y:=\frac{1}{2}(x y+y x)
$$

É claro que uma álgebra A é associativa (comutativa) se e somente se o associador (comutador) de 3 (2) elementos arbitrários da álgebra é sempre zero.

Adotando esta notação, temos que uma álgebra A é alternativa se e só se nela são satisfeitas as equações $(x, x, y)=(x, y, y)=0$, para quaisquer $x, y \in A$.

A seguir enunciaremos uma versão particular de um resultado bem mais geral que o leitor interessado pode encontrar em [12, p.12].

Proposição 2 Se A é uma álgebra alternativa sobre $\Phi$ e $f \in \Phi[X]$ é uma identidade em $A$ então todas as linearizações parciais $\Delta_{i}^{k} f$ também são identidades em $A$. 
Usando esta proposição, é fácil demonstrar ([12, p.35]) que, na variedade das álgebras alternativas as seguintes relações são satisfeitas:

$x(y x)=(x y) x$

$x(y z y)=[(x y) z] y$

$(y z y) x=y[z(y x)]$

$(x y)(z x)=x(y z) x$

(lei flexível)

(identidade direita de Moufang) (identidade esquerda de Moufang)

(identidade média de Moufang)

Notemos que, pela lei flexível, não é preciso pôr parênteses no lado direito da equação da identidade média de Moufang, nem no lado esquerdo das identidades direita e esquerda de Moufang. Novamente, usando as linearizações, prova-se que em toda álgebra alternativa o associador, como função de 3 variáveis, é antissimétrico em seus argumentos e valem as identidades:

$$
\begin{array}{r}
(x, x y, z)=(x, y, z) x \\
(x, y x, z)=x(x, y, z) \\
\left(x^{2}, y, z\right)=(x, x \circ y, z) \\
\left(x^{2}, y, z\right)=x \circ(x, y, z)
\end{array}
$$

Mediante estas igualdades e usando indução, não é difícil provar um conhecido resultado devido a Artin que enunciamos a seguir. A prova pode ser vista em [12, p.36].

Teorema 1 (Artin) Numa álgebra alternativa $A$ dois elementos quaisquer geram uma subálgebra associativa.

Corolário 1 Toda álgebra alternativa é de potências associativas.

Agora introduzimos um polinômio $f$, de 4 variáveis, chamado geralmente de função de Kleinfeld, a qual nos será útil, pois graças a suas propriedades, muitas vezes logramos simplificar os cálculos efetuados em álgebras alternativas:

$$
f(w, x, y, z):=(w x, y, z)-x(w, y, z)-(x, y, z) w
$$

Lema 2 Em toda álgebra alternativa, $f(w, x, y, z)$ é uma função antissimétrica em seus argumentos. 
A demonstração deste lema segue da linearidade de $f$ em cada variável e de que, devido às identidades de Moufang e às equações (1.1),..,(1.4), $f(w, x, y, z)=0$ cada vez que dois de seus argumentos são iguais.

Lema 3 Em toda álgebra alternativa é satisfeita a seguinte igualdade:

$$
f(w, x, y, z)=([w, x], y, z)+([y, z], w, x)
$$

Este resultado é obtido fundamentalmente a partir da linearização parcial de $(x y, x, y)=0$ em ambas as variáveis, identidade que segue do teorema de Artin.

\section{3 Álgebras de composição}

Uma das formas naturais em que aparecem as álgebras alternativas é mediante o estudo das álgebras de composição, as quais surgem como uma generalização natural das álgebras dos números complexos, dos quatérnios e da álgebra de Cayley-Dickson. É porisso que dedicamos esta seção a enunciar os principais resultados, assim como a dar um esboço do processo de Cayley-Dickson mediante o qual são construidas álgebras de composição a partir de um corpo $F$ arbitrário. As provas podem ser encontradas em [12, p.22-p.48].

Definição 3 Seja $A$ uma $F$-álgebra. Uma aplicação $n: A \longrightarrow F$ é chamada uma forma quadrática, se satisfaz:

(1) $n(\lambda x)=\lambda^{2} n(x)$, onde $x \in A$ e $\lambda \in F$.

(2) $f(x, y)=n(x+y)-n(x)-n(y)$ é uma forma bilinear em A.

Chamaremos a forma quadrática $n(x)$ de estritamente não degenerada se a forma bilinear simétrica $f(x, y)$ associada a ela é não degenerada, ou seja, se $f(a, x)=0$ para todo $x \in A$ implica que $a=0$. Diremos também que a forma $n(x)$ admite composição se existe uma operação binária em $A$ (composição) que verifica $n(x y)=n(x) n(y)$, para quaisquer $x, y \in A$.

Definição 4 Uma álgebra $A$ sobre um corpo $F$ com uma forma quadrática $n(x)$ é chamada álgebra de composição, se são satisfeitas as seguintes condições:

(1) $n(x y)=n(x) n(y)$, para todo $x, y \in A$

(2) $n(x)$ é uma forma estritamente não degenerada.

(3) $A$ contém um elemento unidade 1. 
Diremos que um endomorfismo $\rho$ do espaço vetorial $A$ é uma involução se $\rho(\rho(a))=a$ e $\rho(a b)=\rho(b) \rho(a)$, para quaisquer $a, b \in A$. Nestas condições, é fácil provar que toda álgebra de composição é alternativa. Além disso, a aplicação $a \longrightarrow \bar{a}=f(1, a)-a$ é uma involução que fixa os elementos do corpo. Além do mais, se definimos a aplicação traço que associa a cada elemento $a \in A$ o elemento $\operatorname{tr}(a)=a+\bar{a}$ então $\operatorname{tr}: A \longrightarrow F, n(a)=a \bar{a}$ e cada elemento da álgebra satisfaz a equação $a^{2}-\operatorname{tr}(a) a+n(a)=0$. Portanto, toda álgebra de composição é quadrática sobre o corpo onde está definida.

A seguir, consideraremos uma álgebra $A$ sobre um corpo $F$ com 1 e com involução $a \longrightarrow \bar{a}$, onde $a+\bar{a}$ e $a \bar{a} \in F$ para todo $a \in A$. Entenderemos por processo de Cayley-Dickson a construção de uma nova álgebra com involução que contém $A$ como subálgebra e com dimensão duas vezes a dimensão de $A$.

Fixemos $0 \neq \alpha \in F$ e denotemos por $(A, \alpha)$ o conjunto de todos os pares ordenados $(a, b)$, onde $a$ e $b \in A$. Definamos em $(A, \alpha)$ a soma e multiplicação por escalar componente a componente e também a seguinte regra de multiplicação:

$$
\left(a_{1}, a_{2}\right)\left(a_{3}, a_{4}\right)=\left(a_{1} a_{3}+\alpha a_{4} \bar{a}_{2}, \bar{a}_{1} a_{4}+a_{3} a_{2}\right)
$$

Com as operações assim definidas, $(A, \alpha)$ é uma álgebra sobre $F$, o elemento $(1,0)$ é uma unidade em $(A, \alpha)$ e o conjunto $A^{\prime}=\{(a, 0) \mid a \in A\}$ é uma subálgebra de $(A, \alpha)$ que é isomorfa à álgebra $A$. Além disso, se escrevemos $v=(0,1)$, temos que $v^{2}=\alpha(1,0)$ e $(A, \alpha)$ é soma direta dos espaços vetoriais $A^{\prime}$ e $v A^{\prime}$. Identificando $A^{\prime}$ com $A$ podemos representar os elementos de $(A, \alpha)$ na forma $x=a_{1}+v a_{2}$, onde $a_{1}, a_{2} \in A$ estão unicamente determinados pelo elemento $x$. Nesta representação, a multiplicação em $(A, \alpha)$ está dada por

$$
\left(a_{1}+v a_{2}\right)\left(a_{3}+v a_{4}\right)=\left(a_{1} a_{3}+\alpha a_{4} \bar{a}_{2}\right)+v\left(\bar{a}_{1} a_{4}+a_{3} a_{2}\right) .
$$

Se para cada $x=a_{1}+v a_{2} \in(A, \alpha)$ definimos $\bar{x}=\bar{a}_{1}-v a_{2}$ temos o seguinte resultado:

Lema $4 A$ aplicação $x \longrightarrow \bar{x}$ é uma involução da álgebra $(A, \alpha)$. Além do mais, para todo $x \in A$ temos que $x+\bar{x}$ e $x \bar{x} \in F$ e se a forma quadrática $n(a)=a \bar{a}$ é estrictamente não degenerada em $A$, então a forma quadrática $n(x)=x \bar{x}$ é estritamente não degenerada em $(A, \alpha)$.

Agora, se consideramos uma álgebra de composição $A$ podemos aplicar o processo anterior e obter uma nova álgebra $(A, \alpha)$. A álgebra obtida é 
uma álgebra de composição se e somente se a álgebra $A$ é associativa. Desta forma, a partir do corpo $F$, podemos construir alguns exemplos de álgebras de composição que listamos a seguir:

i) Se $F$ é um corpo de característica distinta de 2, então, considerando a forma $n(\alpha)=\alpha^{2}$, obtemos a mais simples das álgebras de composição.

ii) Seja $K(\mu)=F+F v$, onde $v_{1}^{2}=v_{1}+\mu$ e $4 \mu+1 \neq 0$, com involução $\overline{\alpha+\beta v_{1}}=(\alpha+\beta)-\beta v_{1}$ e forma quadrática $n(a)=a \bar{a}$. Então, se o polinômio $x^{2}-x-\mu$ é irredutível em $F[x], K(\mu)$ é um corpo. Caso contrário, $K(\mu)=$ $F \oplus F$. Se consideramos $F$ com característica diferente de 2, então o elemento $v=v_{1}-\frac{1}{2}$ satisfaz $v^{2}=\alpha$, onde $\alpha=\frac{1}{4}(4 \mu+1) \neq 0$ obtendo $K(\mu)=(F, \alpha)$. iii) $Q(\mu, \beta)=(K(\mu), \beta)$ com $\beta \neq 0$, chamada geralmente de álgebra dos quatérnios, a qual é associativa mas não é comutativa.

iv) $C(\mu, \beta, \gamma)$ com $\gamma \neq 0$, conhecida como a álgebra de Cayley-Dickson.

A álgebra de Cayley-Dickson não é comutativa nem associativa. Daí, pelo que afirmamos acima não podemos seguir obtendo álgebras de composição com este processo. Porém, não temos perdido nada já que toda álgebra de composição é isomorfa a alguma álgebra das citadas nos exemplos anteriores.

Daremos especial atenção à álgebra de Cayley-Dickson $C$ pois será usada no último capítulo. Esta álgebra é também conhecida como álgebra matricial de Cayley-Dickson. Isto, pois os elementos de $C$ podem ser representados em forma matricial considerando todas as matrizes da forma $\left(\begin{array}{cc}\alpha & u \\ v & \beta\end{array}\right)$,

onde $\alpha, \beta \in F$ e $u, v \in F^{3}$, com a soma e multiplicação por escalar habituais e com a seguinte regra de multiplicação entre matrizes:

$$
\left(\begin{array}{cc}
\alpha & u \\
v & \beta
\end{array}\right)\left(\begin{array}{cc}
\gamma & z \\
w & \delta
\end{array}\right)=\left(\begin{array}{cc}
\alpha \gamma+(u, w) & \alpha z+\delta u-v \times w \\
\gamma v+\beta w+u \times z & \beta \delta+(v, z)
\end{array}\right)
$$

onde $(a, b)$ denota o produto escalar dos vetores $a$ e $b \in F^{3}$ e $a \times b$ denota o produto vetorial entre $a$ e $b$. Desta forma, definindo a involução $\overline{\left(\begin{array}{ll}\alpha & u \\ v & \beta\end{array}\right)}=$ $\left(\begin{array}{cc}\alpha & -u \\ -v & \beta\end{array}\right)$ e, para o elemento $a=\left(\begin{array}{cc}\alpha & u \\ v & \beta\end{array}\right), n(a)=a \bar{a}=\alpha \beta-(u, v)$, $t(a)=a+\bar{a}=\alpha+\beta$, obtemos realmente uma representação dos elementos da álgebra de Cayley-Dickson. 


\section{Capítulo 2}

\section{Centros de álgebras alternativas}

\subsection{Elementos do centro associativo}

Neste parágrafo vamos encontrar várias novas identidades das álgebras alternativas bem como elementos que pertencem ao centro associativo das álgebras desta variedade.

Definição 5 Dada uma álgebra A sobre o anel $\Phi$ chamamos de centro associativo de $A$ o submódulo $N_{A}=\{n \in A$ tais que $(n, x, y)=(x, n, y)=$ $(x, y, n)=0$ para quaisquer $x, y \in A\}$. Chamamos de centro comutativo de $A$ o submódulo $K_{A}=\{k \in A$ tais que $[k, x]=0$ para todo $x \in A\}$ e chamamos de centro de $A$ o submódulo $Z_{A}=K_{A} \cap N_{A}$.

Comecemos com um resultado geral sobre os centros das álgebras em uma variedade homogênea.

Proposição 3 Se $M$ é uma variedade homogênea, então o centro associativo $N_{M}$, o centro comutativo $K_{M}$ e o centro $Z_{M}$ da álgebra livre $\Phi[X]_{M}$ em $M$ são invariantes sob linearizações parciais.

Demonstração: Tomemos um elemento arbitrário $n=n\left(x_{1}, \ldots x_{n}\right) \in N_{M}$. Consideremos $n \Delta_{i}^{k}\left(x_{j}\right)$ a linearização parcial do elemento $n$ na variável $x_{i}$ de grau $k$ e sejam $x_{r}, x_{l} \in X \backslash\left\{x_{1}, \ldots, x_{m}, x_{j}\right\}$. Nestas condições, $\left(n, x_{r}, x_{l}\right)=$ $\left(x_{r}, n, x_{l}\right)=\left(x_{r}, x_{l}, n\right)=0$. Pelo Lema 1 , como $v(x, y, z)=(x, y, z)$ é um monômio trilinear, temos que $0=\left(n, x_{r}, x_{l}\right) \Delta_{i}^{k}\left(x_{j}\right)=\left(n \Delta_{i}^{k}\left(x_{j}\right), x_{r}, x_{l}\right)$, pois $x_{m} \Delta_{i}^{t}\left(x_{j}\right)=0$ para $m=r, l$ e $t \geq 1$. Do mesmo modo, temos que 
$\left(x_{r}, n \triangle_{i}^{k}\left(x_{j}\right), x_{l}\right)=\left(x_{r}, x_{l}, n \triangle_{i}^{k}\left(x_{j}\right)\right)=0$, ou seja, $n \triangle_{i}^{k}\left(x_{j}\right) \in N_{M}$ e portanto $N_{M}$ é invariante sob linearizações parciais. Analogamente, se consideramos $v(x, y)=[x, y]$ e aplicamos novamente o Lema 1 , concluímos que $K_{M}$ é invariante sob linearizações parciais. Finalmente, como consequência do anterior, $N_{M} \cap K_{M}=Z_{M}$ também é invariante.

Lema 5 Em toda álgebra alternativa $A$ são satisfeitas as seguintes igualdades (de grau 5):

$$
\begin{gathered}
(x, y,(x, y, z))=[x, y](x, y, z) \\
((x, y, z), x, y)=-(x, y, z)[x, y] \\
(x, y, z) \circ[x, y]=0
\end{gathered}
$$

Demonstração: Primeiro notemos que se $x, y, z \in A$ então

$$
\begin{aligned}
(x y)(x, y, z)-y[x(x, y, z)]= & (x y)[(x y) z]-(x y)[x(y z)] \\
& -y[x\{(x y) z\}]+y[x\{x(y z)\}]
\end{aligned}
$$

Se linearizamos a identidade esquerda de Moufang obtemos

$$
(z v u) w+(u v z) w=u[v(z w)]+z[v(u w)]
$$

onde os parênteses em $z v u$ e uvz estão ambos no mesmo lado. Daqui, colocando $u=x y, v=x, z=y, w=z$ e substituindo no segundo termo do lado direito da primeira equação e depois aplicando a identidade esquerda de Moufang obtemos:

$$
\begin{aligned}
(x y)(x, y, z)-y[x((x, y, z)] & =(x y)[(x y) z]-[(x y) x y+y x(x y)] z+y[x\{x(y z)\}] \\
& =(x y)^{2} z-(x y)^{2} z-[y x(x y)] z+y[x(x\{y z\})] \\
& =-[(y x)(x y)] z+y\left[x^{2}(y z)\right] \\
& =-\left(y x^{2} y\right) z+\left(y x^{2} y\right) z=0
\end{aligned}
$$

o que prova que

$$
(x y)(x, y, z)=y[x(x, y, z)]
$$

Agora usando (2.4), temos:

$$
\begin{aligned}
(y, x,(x, y, z)) & =(y x)(x, y, z)-y[x(x, y, z)] \\
& =(y x)(x, y, z)-(x y)(x, y, z) \\
& =[y, x](x, y, z)
\end{aligned}
$$


Daqui, pela antissimetria do associador e do comutador, fica provada a equação (2.1). Analogamente,

$$
\begin{aligned}
((x, y, z), x, y) & =[(x, y, z) x] y-(x, y, z)(x y) \\
& =(x, y, z)[(y x)-(x y)] \\
& =(x, y, z)[y, x] \\
& =-(x, y, z)[x, y]
\end{aligned}
$$

e portanto (2.2) é satisfeita. Finalmente de (2.1) e (2.2), temos:

$$
\begin{aligned}
(x, y, z) \circ[x, y] & =(x, y, z)[x, y]+[x, y](x, y, z) \\
& =-((x, y, z), x, y)-(y, x,(x, y, z)) \\
& =0 .
\end{aligned}
$$

o que prova (2.3) e o lema.

Se denotamos por $(A, s, t)$ o conjunto formado por todos os elementos da forma $(a, s, t)$ com $a \in A$, o seguinte resultado é válido:

Lema 6 Sejam $x, y \in A$ e $B$ a subálgebra de $A$ gerada por $x$ e $y$. Então, para quaisquer $u, v \in B$, temos que $(A, u, v) \subseteq(A, x, y)$.

Demonstração: Pelo teorema de Artin é suficiente prová-lo para palavras associativas $u, v \in B$. Procederemos por indução sobre $n=d(u)+d(v)$. Se $n=2$ o resultado é claro. Seja $n=k$ e suponhamos que o resultado é válido para $n<k$. Daqui em diante escreveremos $r \equiv s$ se $r-s \in(A, x, y)$. Notemos que se $z \in A$ então aplicando a identidade esquerda de Moufang, temos

$$
\begin{aligned}
(z, x, y x y)-(z, x y x, y) & =(z x)(y x y)-z[x(y x y)]-[z(x y x)] y+z[(x y x) y] \\
& =(z x)(y x y)-[z(x y x)] y \\
& =[[(z x) y] x] y-[[(z x) y] x] y \\
& =0
\end{aligned}
$$

portanto

$$
(z, x, y x y)=(z, x y x, y)
$$

Linearizando (2.5) temos

$$
(z, t, y x y)=(z,\{x y t\}, y)+(z, y t y, x)
$$


onde $\{x y t\}:=(x y) t+(t y) x$. Agora consideraremos dois casos.

$1^{0}$ Caso: $u$ ou $v$ começam e terminam com o mesmo elemento (por exemplo $\left.u=x u_{1} x\right)$. Então, se $d\left(u_{1}\right) \geq 1$, usando (2.6) e a hipótese de indução segue que

$$
\begin{aligned}
(z, u, v) & =\left(z, x u_{1} x, v\right) \\
& =\left(x z x, u_{1}, v\right)+\left(\left\{z x u_{1}\right\}, x, v\right) \\
& \equiv 0
\end{aligned}
$$

pois $d\left(u_{1}\right)+d(v)<k$ e $d(x)+d(v)<k$. Se $d\left(u_{1}\right)=0$, ou seja, se $u=x^{2}$, então pela hipótese de indução e (1.3) temos $(z, u, v)=\left(z, x^{2}, v\right)=$ $(z \circ x, x, v) \equiv 0$, pois $d(x)+d(v)<k$.

$2^{0}$ Caso: O começo ou o final de $u$ é igual ao começo de $v$, por exemplo, $u=x u_{1}, v=x v_{1}$. Neste caso $\left(z, x u_{1}, x v_{1}\right)=-\left(z, x u_{1}, v_{1} x\right)+2\left(z, x u_{1}, x \circ v_{1}\right)$. Ao linearizar a equação de Moufang $(x \circ y, x, z)=\left(y, x^{2}, z\right)$ (eq. (1.3)) obtemos $(t \circ y, x, z)+(x \circ y, t, z)=(y, t \circ y, z)$. Substituindo $y=v_{1}, t=z \mathrm{e}$ $z=x u_{1}$ nesta identidade podemos concluir, pela hipótese de indução, que

$$
\begin{aligned}
\left(z, x u_{1}, x v_{1}\right) & =-\left(z, x u_{1}, v_{1} x\right)+2\left(z \circ x, x u_{1}, v_{1}\right)+2\left(z \circ v_{1}, x u_{1}, x\right) \\
& \equiv-\left(z, x u_{1}, v_{1} x\right)
\end{aligned}
$$

Por outro lado, $[(z, x, x t)-(x z, x, t)] \Delta_{x}^{1}(y)=0$. Colocando nesta equação $z=z, x=v_{1}, y=x u_{1}, t=x$, usando a hipótese de indução e o primeiro caso, temos $\left(z, x u_{1}, v_{1} x\right)=\left(z, v_{1}, x u_{1} x\right)-\left(v_{1} z, x u_{1}, x\right)-\left(\left(x u_{1}\right) z, v_{1}, x\right) \equiv 0$.

A demonstração no caso em que $u=u_{1} x$ é análoga, ficando provado o lema.

Corolário 2 Seja $A$ uma álgebra alternativa, $x, y, z \in A$ elementos quaisquer, $u_{i}, v_{i}(i=1,2)$ elementos da subálgebra gerada por $x, y$. Vale a seguinte relação:

$$
\left(u_{1}, v_{1}, z\right) \circ\left[u_{2}, v_{2}\right]=0
$$

Demonstração: Pelo teorema de Artin basta provar que, para duas palavras associativas $u(x, y), v(x, y)$ quaisquer, tem-se que

$$
\left(u_{1}, v_{1}, z\right) \circ[u, v]=0
$$

Novamente faremos a demonstração aplicando indução sobre $n=d\left(u_{1}\right)+$ $d\left(u_{2}\right)$. Se $n=2$, pelo Lema $6,\left(u_{1}, v_{1}, z\right)=\left(x, y, z_{1}\right)$, para algum $z_{1} \in A$ 
e portanto, neste caso, o corolário é satisfeito. Consideremos agora $n=$ $k>2$ e suponhamos (2.7) válido para $n<k$. Se $d(u)>1$ e $d(v)=1$ ( $v=x$, por exemplo), então observemos que linearizando (2.3) obtemos $0=((x, y, z) \circ[x, y]) \Delta_{y}^{1}=(x, y, z) \circ[u, x]+(u, y, z) \circ[x, y]$. Pela hipótese de indução concluímos que

$$
\begin{aligned}
\left(u_{1}, v_{1}, z\right) \circ[u, x] & =\left(x, y, z_{1}\right) \circ[u, x] \\
& =-\left(u, y, z_{1}\right) \circ[x, y]
\end{aligned}
$$

Se $d(u)>1$ e $d(v)>1$, analogamente ao caso anterior mas usando o fato que $\left[((x, y, z) \circ[x, y]) \Delta_{y}^{1}(u)\right] \Delta_{x}^{1}(v)=0$, temos

$$
\begin{aligned}
\left(u_{1}, v_{1}, z\right) \circ[u, v] & =\left(x, y, z_{1}\right) \circ[u, v] \\
& =-\left(u, y, z_{1}\right) \circ[x, v]-\left(x, v, z_{1}\right) \circ[u, x] \\
& =0
\end{aligned}
$$

o que prova o corolário.

Observemos que este resultado é uma generalização da equação (2.3).

Teorema 2 Sejam A uma álgebra alternativa, $x, y \in A$ dois elementos quaisquer e $B$ a subálgebra gerada por $x, y$. Então, se $u_{i}, v_{i} \in B, r, s \in A e$ $w_{i}=\left[u_{i}, v_{i}\right]$, temos as seguintes igualdades:

$$
\begin{aligned}
\left(w_{1} \circ w_{2}, r, s\right) w_{3} & =w_{3}\left(w_{1} \circ w_{2}, r, s\right)=0 \\
\left(w_{1}^{2}, r, s\right) w_{2} & =w_{2}\left(w_{1}^{2}, r, s\right)=0 \\
\left(w_{1}^{2}\left(w_{2} \circ w_{3}\right), r, s\right) & =\left(\left(w_{2} \circ w_{3}\right) w_{1}^{2}, r, s\right)=0 \\
\left(w_{1}^{2} w_{2}^{2}, r, s\right) & =0
\end{aligned}
$$

Demonstração: Para começar, vamos provar que se $r \in A$ e $u \in B$ então

$$
\left(w_{1} \circ w_{2}, u, r\right)=\left(w_{1}^{2}, u, r\right)=0
$$

Com efeito, usando a linearização parcial de (1.4) junto com (2.7), temos $\left(w_{1} \circ w_{2}, u, r\right)=w_{1} \circ\left(w_{2}, u, r\right)+w_{2} \circ\left(w_{1}, u, r\right)=0$ e, além disso, $\left(w_{1}^{2}, u, r\right)=$ $w_{1}\left(w_{1}, u, r\right)=0$. Linearizando em $\mathrm{x}$ e y a identidade (2.2) obtemos:

$$
\begin{aligned}
& ((t, w, z), x, y)+((t, y, z), x, w)+((x, w, z), t, y)+((x, y, z), t, w)+ \\
& \quad(x, w, z)[t, y]+(x, y, z)[t, w]+(t, w, z)[x, y]+(t, y, z)[x, w]=0
\end{aligned}
$$


Substituindo $z=w_{1} \circ w_{2}, x=u_{3}, w=s, t=r, y=v_{3}$ em (2.14) e observando que o $1^{0}, 2^{0}, 4^{0}$ e $5^{0}$ termos se anulam por causa de (2.13), e o $3^{0}$ e $7^{0}$ pelo teorema de Artin, temos que

$$
\begin{aligned}
\left(w_{1} \circ w_{2}, r, s\right) w_{3}= & \left(w_{1} \circ w_{2}, r, s\right)\left[u_{3}, v_{3}\right] \\
= & -\left(w_{1} \circ w_{2}, u_{3}, s\right)\left[r, v_{3}\right]-\left(w_{1} \circ w_{2}, r, v_{3}\right)\left[u_{3}, s\right] \\
& -\left(w_{1} \circ w_{2}, u_{3}, v_{3}\right)[r, s]-\left(\left(w_{1} \circ w_{2}, r, s\right), u_{3}, v_{3}\right) \\
& -\left(\left(w_{1} \circ w_{2}, u_{3}, s\right), r, v_{3}\right)-\left(\left(w_{1} \circ w_{2}, r, v_{3}\right), u_{3}, s\right) \\
& -\left(\left(w_{1} \circ w_{2}, u_{3}, v_{3}\right), r, s\right) \\
= & -\left(\left(w_{1} \circ w_{2}, r, s\right), u_{3}, v_{3}\right)
\end{aligned}
$$

Além do mais, linearizando (1.4), obtemos a seguinte igualdade:

$$
(x \circ t, y, z)-x \circ(t, y, z)-t \circ(x, y, z)=0
$$

de onde segue que $\left(\left(w_{1} \circ w_{2}, r, s\right), u_{3}, v_{3}\right)=\left(w_{1} \circ\left(w_{2}, r, s\right), u_{3}, v_{3}\right)+\left(w_{2} \circ\right.$ $\left.\left(w_{1}, r, s\right), u_{3}, v_{3}\right)$. Aplicando novamente (2.15), (2.7) e o teorema de Artin, temos:

$$
\begin{aligned}
\left(\left(w_{1} \circ w_{2}, r, s\right), u_{3}, v_{3}\right)= & w_{1} \circ\left(\left(w_{2}, r, s\right), u_{3}, v_{3}\right)+\left(w_{2}, r, s\right) \circ\left(w_{1}, u_{3}, v_{3}\right) \\
& +w_{2} \circ\left(\left(w_{1}, r, s\right), u_{3}, v_{3}\right)+\left(w_{1}, r, s\right) \circ\left(w_{2}, u_{3}, v_{3}\right) \\
= & 0
\end{aligned}
$$

e portanto, $\left(w_{1} \circ w_{2}, r, s\right) w_{3}=0$. Analogamente, usando a linearização parcial de (1.1) em vez de (1.2), conclui-se que $w_{3}\left(w_{1} \circ w_{2}, r, s\right)=0$, ficando provada assim a equação (2.9). Notemos que se a característica de $\mathrm{F}$ é diferente de 2, então (2.10) é consequência direta de (2.9), assim como (2.12) de (2.11). Como não temos esta hipótese, substituimos $z=w_{1}^{2}, x=r, w=s, t=u$, $y=v_{2}$ em (2.14). Usando (2.13), o teorema de Artin e (1.4) temos que

$$
\begin{aligned}
-\left(w_{1}^{2}, r, s\right) w_{2}= & -\left(w_{1}^{2}, r, s\right)\left[u_{2}, v_{2}\right] \\
= & \left(\left(u_{2}, s, w_{1}^{2}\right), r, v_{2}\right)+\left(\left(u_{2}, v_{2}, w_{1}^{2}\right), r, s\right)+\left(\left(r, s, w_{1}^{2}\right), u_{2}, v_{2}\right) \\
& +\left(\left(r, v_{2}, w_{1}^{2}\right), u_{2}, s\right)+\left(r, v_{2}, w_{1}^{2}\right)\left[u_{2}, s\right]+\left(u_{2}, s, w_{1}^{2}\right)\left[r, v_{2}\right] \\
& +\left(u_{2}, v_{2}, w_{1}^{2}\right)[r, s] \\
= & \left(\left(r, s, w_{1}^{2}\right), u_{2}, v_{2}\right) \\
= & \left(\left(r, s, w_{1}\right) \circ w_{1}, u_{2}, v_{2}\right) \\
= & \left(r, s, w_{1}\right) \circ\left(w_{1}, u_{2}, v_{2}\right)+w_{1} \circ\left(\left(r, s, w_{1}\right), u_{2}, v_{2}\right)=0
\end{aligned}
$$

e analogamente, $w_{2}\left(w_{1}^{2}, r, s\right)=0$, portanto (2.10) é satisfeita. Para demonstrar (2.11) consideremos a função $f$ de Kleinfeld. De (2.13) segue que

$f\left(r, s, w_{1}^{2}, w_{2} \circ w_{3}\right)=\left(r s, w_{1}^{2}, w_{2} \circ w_{3}\right)-s\left(r, w_{1}^{2}, w_{2} \circ w_{3}\right)-\left(r, w_{1}^{2}, w_{2} \circ w_{3}\right) s=0$ 
Como $f$ é uma função antissimétrica em seus argumentos, fazendo uso das identidades esquerda e direita de Moufang e também de (2.9) e (2.10) temos:

$$
\begin{aligned}
\left(w_{1}^{2}\left(w_{2} \circ w_{3}\right), r, s\right)= & f\left(w_{1}^{2}, w_{2} \circ w_{3}, r, s\right)+\left(w_{2} \circ w_{3}\right)\left(w_{1}^{2}, r, s\right) \\
& +\left(w_{2} \circ w_{3}, r, s\right) w_{1}^{2} \\
= & w_{2}\left(w_{3}\left(w_{1}^{2}, r, s\right)\right)+w_{3}\left(w_{2}\left(w_{1}^{2}, r, s\right)\right) \\
& +\left(\left(w_{2} \circ w_{3}, r, s\right) w_{1}\right) w_{1} \\
= & 0
\end{aligned}
$$

Analogamente, $\left(\left(w_{2} \circ w_{3}\right) w_{1}^{2}, r, s\right)=0$, ficando provada (2.11). Finalmente, em vista do anterior.

$$
\begin{aligned}
\left(w_{1}^{2} w_{2}^{2}, r, s\right) & =f\left(w_{1}^{2}, w_{2}^{2}, r, s\right)+w_{1}^{2}\left(w_{2}^{2}, r, s\right)+w_{2}^{2}\left(w_{1}^{2}, r, s\right) \\
& =w_{1}\left(w_{1}\left(w_{2}^{2}, r, s\right)\right)+w_{2}\left(w_{2}\left(w_{1}^{2}, r, s\right)\right) \\
& =0
\end{aligned}
$$

Portanto (2.12) e o Teorema 2 estão provados.

Corolário 3 Em toda álgebra alternativa são satisfeitas as seguintes relaçôes:

$$
\begin{aligned}
& {[x, y]\left([x, y]^{2}, r, s\right)=0} \\
& \left([x, y]^{2}, r, s\right)[x, y]=0 \\
& \left([x, y]^{4}, r, s\right)=0
\end{aligned}
$$

Notemos que a terceira identidade do Corolário 3 nos diz que, para todo $x, y$ na álgebra alternativa $\mathrm{A}$, o elemento $[x, y]^{4} \in N(A)$.

Corolário 4 Os elementos $n_{i}\left(x_{1}, x_{2}\right)$ definidos por $n_{1}\left(x_{1}, x_{2}\right)=\left[x_{1}, x_{2}\right]^{4}$, $n_{2}\left(x_{1}, x_{2}\right)=\left[x_{1}, x_{2}\right]^{2}\left(\left[x_{1}, x_{2}\right] \circ\left[x_{1}, x_{2} x_{1}\right]\right)$ e $n_{3}\left(x_{1}, x_{2}\right)=\left[x_{1}, x_{2}\right]^{2}\left[x_{1}, x_{2} x_{1}\right]^{2}$ pertencem a $N_{\text {alt }}$ (o centro associativo da álgebra livre $\Phi[X]_{\text {Alt }}$ na variedade das álgebras alternativas) e a seguinte relação é satisfeita

$$
n_{1}\left(x_{1}, x_{2}\right) x_{1}^{2}+n_{2}\left(x_{1}, x_{2}\right) x_{1}+n_{3}\left(x_{1}, x_{2}\right)=0
$$

A demonstração deste corolário segue simplesmente do desenvolvimento da equação. Devemos notar que, em certa forma, pelo Corolário 4, toda álgebra alternativa é quadrática sobre seu centro associativo, pois ao desenvolver as expressões que definem $n_{i}, i=1,2,3$, vemos que estes são não nulos em Ass[X] e, portanto, também são não nulos em Alt $[\mathrm{X}]$. Em particular, temos que $N_{\text {Alt }[X]} \neq(0)$. 


\subsection{Elementos do centro comutativo}

Em seguida provaremos um resultado que pode ser interpretado como um dual ao visto no Corolário 3. Numa álgebra alternativa A que não contém elementos de ordem aditiva igual a 2 , para todo $x, y, z \in A$, temos $(x, y, z)^{4} \in$ $K(A)$.

Para facilitar a compreensão, listaremos a seguir algumas das identidades que já temos provado e que usaremos frequentemente no decorrer desta seção

$$
\begin{aligned}
{[x, y] \circ(x, y, z) } & =0 \\
(x, y, z)[y, z]+((x, y, z), y, z) & =0 \\
{[(x, y, z),[y, z]]+2((x, y, z), y, z) } & =0 \\
\left(x^{2}, y, z\right)=(x, x \circ y, z) & =x \circ(x, y, z) \\
{\left[x^{2}, y\right]=[x, x \circ y] } & =x \circ[x, y]
\end{aligned}
$$

Observação 1 No caso que as igualdades sejam entre 3 termos, como em (2.19) e (2.20), antepomos um E se aludimos à igualdade entre os membros da esquerda, um $D$ aos da direita e a ausência de tais letras indicará que se está fazendo alusão à igualdade entre os termos dos extremos.

Daqui em diante, ao escrever $\mathrm{A}$, estaremos nos referindo a uma álgebra alternativa sem elementos de ordem aditiva igual a 2 . Isto é, supomos que $2 a=0$ implica $a=0, a \in A$.

Lema 7 Sejam $x, y, z, r$ e $t \in A$ e seja $w=(x, y, z)$. Então em $A$ são satisfeitas as seguintes identidades:

$$
\begin{aligned}
{\left[w^{2}, x\right] } & =0 \\
\left(w^{2}, x, y\right) & =0 \\
4 w^{2} & =w \circ[x,[z, y]]
\end{aligned}
$$

Demonstração: Vamos começar demonstrando que

$$
\left[\left(x^{2}, y, z\right),(x, y, z)\right]=0
$$

Com efeito, linearizando (1.1) obtemos

$$
(u, v w, r)+(u, r w, v)=(u, w, r) v+(u, w, v) r
$$


Aplicando esta identidade 2 vezes junto com (E2.19), temos:

$$
\begin{aligned}
\left(x^{2}, y, z\right)(x, y, z)= & -\left(x^{2}, y,(x, y, z)\right) z+\left(x^{2},(x, y, z) y, z\right) \\
& +\left(x^{2}, z y,(x, y, z)\right) \\
= & -(x, y, x \circ(x, y, z)) z+(x, x \circ(x, y, y z), z) \\
& +(x, z y,(x, y, z) \circ x) \\
= & -\left(x, y,\left(x^{2}, y, z\right)\right) z+\left(x,\left(x^{2}, y, y z\right), z\right) \\
& +\left(x, z y,\left(x^{2}, y, z\right)\right) \\
= & (x, y, z)\left(x^{2}, y, z\right)
\end{aligned}
$$

concluindo (2.24). Agora de (E2.20), (2.19) e (2.24), temos

$$
\left[w^{2}, x\right]=[w, w \circ x]=\left[(x, y, z),\left(x^{2}, y, z\right)\right]=0
$$

portanto (2.21) é válido. Por outra parte, de (2.19), (2.17) e (2.16),

$$
\begin{aligned}
& \left(w^{2}, x, y\right)=w \circ(w, x, y) \quad=-w \circ(w[x, y]) \\
& =-w^{2}[x, y]-w[x, y] w=-w([x, y] \circ w)=0
\end{aligned}
$$

e daqui, (2.22) está provada. Se agora usamos (2.19), (2.18), (E2.19) e as linearizações de (2.19) e (2.20), obtemos

$$
\begin{aligned}
4 w^{2} & =2(x, y, z) \circ(x, y, z) \\
& =2\left(\left(x^{2}, y, z\right), y, z\right)-2 x \circ((x, y, z), y, z) \\
& =-\left[\left(x^{2}, y, z\right),[y, z]\right]+x \circ([(x, y, z),[y, z]]) \\
& =-w \circ[x,[z, y]] \\
& =w \circ[x,[z, y]]
\end{aligned}
$$

portanto (2.23) e o Lema 7 estão provados.

Lema 8 Mantendo a notação do lema anterior, a seguinte equação é satisfeita:

$$
w\left(w^{2}, x, r\right)=\left(w^{2}, x, r\right) w=0
$$

Demonstração: É claro que basta provar que as seguintes identidades são satisfeitas:

$$
\begin{aligned}
& {\left[w,\left(w^{2}, x, r\right)\right]=0} \\
& w \circ\left(w^{2}, x, r\right)=0
\end{aligned}
$$


De (1.1), (1.2), (1.3) e (2.21), temos

$$
\begin{aligned}
{\left[w,\left(w^{2}, x, r\right)\right] } & =w\left(w^{2}, x, r\right)-\left(w^{2}, x, r\right) w \\
& =\left(w^{2}, x w, r\right)-\left(w^{2}, w x, r\right) \\
& =\left(w^{2},[x, w], r\right)=(w, w \circ[x, w], r) \\
& =\left(w,\left[x, w^{2}\right], r\right) \\
& =0
\end{aligned}
$$

Em seguida demonstraremos (2.27). Para isto, comecemos usando (2.23), a linearização de (2.20), (2.16) e a linearização de (E2.19):

$$
\begin{aligned}
4 w \circ\left(w^{2}, x, r\right) & =w \circ(w \circ[x,[z, y]], x, r) \\
& =w \circ([x, w \circ[z, y] \circ x, r)-w \circ([z, y] \circ[w, x], x, r) \\
& =-w \circ([z, y] \circ[w, x], x, r)
\end{aligned}
$$

Além disso, usando a linearização parcial de (2.19) junto com (D2.19) e (2.20), temos:

$$
\begin{aligned}
-w \circ([z, y] \circ[w, x], x, r)= & -w \circ([w, x],[z, y] \circ x, r) \\
& -w \circ([z, y], x \circ[w, x], r) \\
= & -w \circ([w, x],[z, y] \circ x, r) \\
& -w \circ\left([z, y],\left[w, x^{2}\right], r\right) .
\end{aligned}
$$

Considerando agora a linearização parcial de (2.19) e (2.20), vemos que

$$
\begin{gathered}
w \circ\left([z, y],\left[w, x^{2}\right], r\right)=\left([z, y],\left[w^{2}, x^{2}\right], r\right)-\left[w, x^{2}\right] \circ([z, y], w, r) \\
\text { e } \quad w \circ([w, x],[z, y] \circ x, r)=\left(\left[w^{2}, x\right],[z, y] \circ x, r\right)-[w, x] \circ(w,[z, y] \circ x, r)
\end{gathered}
$$

Daqui, usando (2.20) e (2.21) obtemos:

$$
\begin{aligned}
4 w \circ\left(w^{2}, x, r\right)= & -\left([z, y],\left[w^{2}, x\right] \circ x, r\right)+\left[w, x^{2}\right] \circ([z, y], w, r) \\
= & -\left(\left[w^{2}, x\right],[z, y] \circ x, r\right)+[w, x] \circ(w,[z, y] \circ x, r) \\
= & -\left([z, y],\left[w^{2}, x\right] \circ x, r\right)+\left[w, x^{2}\right] \circ([z, y], w, r) \\
& +[w, x] \circ(w,[z, y] \circ x, r)
\end{aligned}
$$

Considerando agora a linearização parcial de (2.16), (2.20), (2.19) e (E2.19), temos

$$
\begin{aligned}
4 w \circ\left(w^{2}, x, r\right)= & -\left([z, y],\left[w^{2}, x\right] \circ x, r\right)-[w, r] \circ\left([z, y], w, x^{2}\right) \\
& -[[w, r] \circ(w,[z, y] \circ x, x) \\
= & -[w, r] \circ\left\{\left([z, y], w, x^{2}\right)+(w,[z, y] \circ x, x)\right\} \\
= & 0
\end{aligned}
$$


Como em A não existem elementos com ordem aditiva igual a 2, (2.27) fica provado, o que termina a demonstração do Lema 8.

Lema $9 \mathrm{Em} A$ valem as seguintes igualdades:

$$
\begin{aligned}
w\left(t\left(w^{2}, x, r\right)\right) & =-w\left(\left(w^{2}, x, t\right) r\right) \\
\left(w, y,\left(w^{2}, x, r\right)\right) & =0 \\
(y w)\left\{\left(w^{2}, x, r\right) z\right\} & =0
\end{aligned}
$$

Demonstração: Sendo $f$ a função de Kleinfeld, pelo Lema 3 e usando (2.21), temos:

$$
f\left(w^{2}, x, t, r\right)=\left(\left[w^{2}, x\right], t, r\right)+\left(w^{2}, x,[t, r]\right)=\left(w^{2}, x,[t, r]\right)
$$

daqui e pela equação (2.25) temos que $w f\left(w^{2}, x, t, r\right)=f\left(w^{2}, x, t, r\right) w=0$. Usando a linearização parcial de (2.19) e outra vez (2.25), temos

$$
\begin{aligned}
w\left(t\left(w^{2}, x, r\right)\right) & =w\left(w^{2}, x, r t\right)-w\left(\left(w^{2}, x, t\right) r\right)-w f\left(r, t, w^{2}, x\right) \\
& =-w\left(\left(w^{2}, x, t\right) r\right)
\end{aligned}
$$

e daí (2.28) é satisfeita. Se agora colocamos $r=y$ em (2.28) e usamos (2.22) obtemos

$$
w\left\{\left(w^{2}, x, t\right), y\right\}=-w\left(t\left(w^{2}, x, y\right)\right)=0
$$

Desta igualdade e de (2.25), segue que

$$
\begin{aligned}
\left(w, y,\left(w^{2}, x, r\right)\right) & =-\left(w,\left(w^{2}, x, r\right), y\right) \\
& =-\left\{w\left(w^{2}, x, r\right)\right\} y+w\left\{\left(w^{2}, x, r\right) y\right\} \\
& =0
\end{aligned}
$$

portanto (2.29) fica provada. Finalmente das equações (2.29) e (2.25) temos

$$
(y w)\left(w^{2}, x, r\right)=\left(y, w,\left(w^{2}, x, r\right)\right)+y\left\{w\left(w^{2}, x, r\right)\right\}=0
$$

Mediante o mesmo esquema obtemos

$$
(y w) f\left(w^{2}, x, r, t\right)=0
$$

e $\quad(y w)\left\{\left(w^{2}, x, r\right) t\right\}=-(y w)\left\{r\left(w^{2}, x, t\right)\right\}$

onde, colocando $t=z$ e fazendo uso de (2.22), deduz-se (2.30). 
Teorema 3 Seja A uma álgebra alternativa sem elementos de ordem aditiva igual a 2. Então são satisfeitas as seguintes igualdades:

$$
(x, y, z)^{2}\left[(x, y, z)^{2}, r\right]=\left[(x, y, z)^{2}, r\right](x, y, z)^{2}=\left[(x, y, z)^{4}, r\right]=0
$$

Demonstração: Se chamamos $w=(x, y, z)$, então linearizando (2.17) nas duas variáveis e usando também (2.22) e (2.21), temos

$$
\begin{aligned}
w\left[w^{2}, r\right]= & (x, y, z)\left[w^{2}, r\right] \\
= & -\left(x, w^{2}, z\right)[y, r]-(x, y, r)\left[w^{2}, z\right] \\
& -\left(x, w^{2}, r\right)[y, z]-\left(\left(x, w^{2}, z\right), y, r\right)-\left(\left(x, w^{2}, r\right), y, z\right) \\
= & -\left(x, w^{2}, r\right)[y, z]-\left((x, y, r), w^{2}, z\right)-\left(\left(x, w^{2}, r\right), y, z\right)
\end{aligned}
$$

de onde segue que

$$
\begin{aligned}
w^{2}\left[w^{2}, r\right]= & -w\left\{\left(x, w^{2}, r\right)[y, z]\right\}-w\left\{\left((x, y, r), w^{2}, z\right)\right\} \\
& -w\left\{\left(\left(x, w^{2}, r\right), y, z\right)\right\}
\end{aligned}
$$

Em seguida provaremos que cada um dos termos do lado direito de (2.31) é nulo. Usando (2.28) e (2.16) temos que

$$
w\left\{\left(x, w^{2}, r\right)[y, z]\right\}=-w\left\{r\left(x, w^{2},[y, z]\right)\right\}=-w\{r(x, w, w \circ[y, z])\}=0
$$

O segundo termo do lado direito de (2.31) anula-se diretamente por (2.25) e finalmente usando a linearização parcial de (1.2) junto com (2.29), (2.25), (2.30), (2.28) e (2.22) temos

$$
\begin{aligned}
w\left(\left(w^{2}, x, r\right), y, z\right) & =-z\left(\left(w^{2}, x, r\right), y, w\right)+\left(\left(w^{2}, x, r\right) w, y, z\right)+\left(\left(w^{2}, x, r\right) z, y, w\right) \\
& =\left(\left(w^{2}, x, r\right) z, y, w\right) \\
& =\left(y, w,\left(w^{2}, x, r\right) z\right) \\
& =(y w)\left\{\left(w^{2}, x, r\right) z\right\}-y\left\{w\left(\left(w^{2}, x, r\right) z\right)\right\} \\
& =y\left\{w\left(\left(w^{2}, x, r\right) z\right)\right\} \\
& =y\left\{w\left(r\left(w^{2}, x, z\right)\right)\right\}=0
\end{aligned}
$$

e assim, provamos que $w^{2}\left[w^{2}, r\right]=0$. Com o mesmo esquema, obtemos $\left[w^{2}, r\right] w^{2}=0$ e, portanto, $\left[w^{4}, r\right]=w^{2} \circ\left[w^{2}, r\right]=w^{2}\left[w^{2}, r\right]+\left[w^{2}, r\right] w^{2}=0$. Portanto concluímos que $(x, y, z)^{4} \in K(A)$, para quaisquer $x, y, z \in A$.

Corolário 5 Nas mesmas condições do Teorema 3 é satisfeita a seguinte identidade:

$$
\left[(x, y, z)^{2}, r\right]^{2}=0
$$


Demonstração: Chamando $w=(x, y, z)$, temos que

$$
\begin{aligned}
2\left[w^{2}, r\right]^{2} & =\left[w^{2}, r\right] \circ\left[w^{2}, r\right] \\
& =\left[w^{2},\left[w^{2}, r\right] \circ r\right]-\left[w^{2},\left[w^{2}, r\right]\right] \circ r \\
& =\left[w^{2},\left[w^{2}, r^{2}\right]\right]=0
\end{aligned}
$$

como em $A$ não existem elementos de ordem aditiva igual a 2, o Corolário 5 fica provado.

\subsection{Centros de álgebras alternativas com 3 geradores}

Nesta seção, consideraremos a álgebra alternativa livre gerada pelo conjunto $X_{3}=\left\{x_{1}, x_{2}, x_{3}\right\}$. Encontraremos elementos não nulos no seu centro associativo $N_{A l t\left[X_{3}\right]}$ e no seu centro $Z_{A l t\left[X_{3}\right]}$. Estes elementos, como se podia esperar, têm uma forma mais simples da encontrada para os elementos nos centros da álgebra $A l t[X]$. Para começar, provemos os seguintes lemas:

Lema 10 Se A é uma álgebra alternativa, as seguintes identidades são satisfeitas em $A$ :

$$
\begin{aligned}
\left([x, y]^{2}, x, z\right) & =0 \\
{\left[(x, y, z)^{2}, x\right] } & =0 \\
\left((x, y, z)^{2}, x, y\right) & =0
\end{aligned}
$$

Demonstração: A identidade (2.32) segue de forma imediata de (2.13). Além do mais, pela equação (2.24) e usando a linearização parcial de (2.20) temos que $\left[(x, y, z)^{2}, x\right]=[(x, y, z),(x, y, z) \circ x]=\left[(x, y, z),\left(x^{2}, y, z\right)\right]=0$, ficando provada (2.33). Por último, aplicando (1.4), (2.2) e (2.3) obtemos $\left(w^{2}, x, y\right)=w \circ(w, x, y)=-w \circ(w[x, y])=-w^{2}[x, y]-w[x, y] w=-w([x, y] \circ$ $w)=0$. Portanto o lema está provado.

Lema 11 (Bruck, Kleinfeld) Se A é uma álgebra alternativa com conjunto de geradores $S$ então um elemento $z \in Z(A)$ se e somente se $[z, S]=(z, S, S)=$ (0).

Demonstração: Numa direção a afirmação é óbvia. Agora consideremos $z \in A$ tal que $[z, S]=(z, S, S)=(0)$ e definamos o conjunto $P=\{p \in A \mid$ 
$(z, p, S)=0\}$. É claro que $P$ é um $\Phi$-submódulo do $\Phi$-módulo $A$. Além disso, se $p$ e $p^{\prime} \in P$ então, pelo Lema 3 :

$$
\begin{aligned}
\left(p p^{\prime}, s, z\right) & =p^{\prime}(p, s, z)+\left(p^{\prime}, s, z\right) p+f\left(p, p^{\prime}, s, z\right) \\
& =f\left(p, p^{\prime}, s, z\right) \\
& =\left(\left[p, p^{\prime}\right], s, z\right)+\left(p, p^{\prime},[s, z]\right) \\
& =\left(\left[p, p^{\prime}\right], s, z\right)
\end{aligned}
$$

Portanto, $\left(p^{\prime} p, s, z\right)=0$ de onde segue que $\left(z, P^{2}, S\right)=(0)$, obtendo assim que $P$ é uma subálgebra de $A$. Mas $S \subset P$, portanto $P=A$. Desta forma provamos que $(z, A, S)=0$. Consideremos agora o conjunto $Q=\{q \in A \mid$ $(z, A, q)=(0)\}$. Como antes, temos que $Q$ é um $\Phi$-submódulo de $A$ e $S \subseteq Q$. Além disso, pelo Lema 2,

$$
\begin{aligned}
\left(q q^{\prime}, a, z\right) & =q^{\prime}(q, a, z)+\left(q^{\prime}, a, z\right) q+f\left(q, q^{\prime}, a, z\right) \\
& =f\left(q, q^{\prime}, a, z\right) \\
& =f\left(q^{\prime}, a, q, z\right) \\
& =\left(q^{\prime} a, q, z\right)-a\left(q^{\prime}, q, z\right)-(a, q, z) q^{\prime} \\
& =0
\end{aligned}
$$

Portanto, $Q^{2} \subseteq Q$, ou seja, $Q$ é uma subálgebra de $A$ e, pelo mesmo argumento anterior, concluímos que $Q=A$ obtendo assim $(z, A, A)=0$, isto é, $z \in N(A)$. Para provar o restante, observemos que, pelo simples desenvolvimento das equações, temos que em toda álgebra é satisfeita a seguinte relação:

$$
[x y, z]-x[y, z]-[x, z] y=(x, y, z)-(x, z, y)+(z, x, y) .
$$

Consideremos agora $R=\{r \in A \mid[z, r]=0\}$. É claro que $R$ é um submódulo de $A$ e se tomamos $r$ e $r^{\prime} \in R$, aplicando a relação anterior e em vista do já feito, obtemos: $\left[r r^{\prime}, z\right]=r\left[r^{\prime}, z\right]+[r, z] r^{\prime}=0$. Portanto, $R^{2} \subseteq R$, ou seja, $R$ é uma subálgebra de $A$ que contém $S$. Daí, $R=A$ e $z \in Z(A)$, com o que fica provado o lema.

Teorema 4 (Dorofeev) Em qualquer álgebra alternativa com 3 geradores são satisfeitas as seguintes identidades:

$$
\begin{gathered}
\left([x, y]^{2}, r, s\right)=0 \\
([x, y] \circ[z, t], r, s)=0
\end{gathered}
$$


Demonstração: É suficiente provar que estas equações são satisfeitas na álgebra alternativa livre $\operatorname{Alt}\left[x_{1}, x_{2}, x_{3}\right]$. Chamemos de $N$ o centro associativo de $\operatorname{Alt}\left[x_{1}, x_{2}, x_{3}\right]$. Primero vamos provar que $\left[x_{1}, x_{2}\right]^{2} \in N$ onde $x_{1}$ e $x_{2}$ são os geradores. Para cada $r, s \in A l t\left[x_{1}, x_{2}, x_{3}\right]$, denotemos por $I(r, s)$ o ideal gerado pelo elemento $\left(\left[x_{1}, x_{2}\right], r, s\right)$ em $A l t\left[x_{1}, x_{2}, x_{3}\right]$. Por outro lado, em toda álgebra alternativa é satisfeita a seguinte equação: $(r, s, x y x)=$ $x(r, s, y) x+x y(r, s, x)$ (que será provada no próximo capítulo), daí e por (1.2), temos que $I(r, s) \subseteq \sum_{i<j} I\left(x_{i}, x_{j}\right)+\sum_{i=1}^{3} I\left(x_{i}, x_{j} x_{k}\right)$ (ver [12, p.261]), onde $i \notin\{j, k\}$. Além do mais, a seguinte igualdade é válida:

$$
I\left(x_{i}, x_{j} x_{k}\right)+\sum_{i<j} I\left(x_{i}, x_{j}\right)=I\left(x_{1}, x_{2} x_{3}\right)+\sum_{i<j} I\left(x_{i}, x_{j}\right) .
$$

A inclusão numa direção é imediata e, na outra, deve ser provada termo a termo, mas como todos os casos são análogos, vamos provar um caso particular. Consideremos $I\left(x_{2}, x_{1} x_{3}\right)$, aplicando a linearização parcial de (1.1) obtemos:

$$
\begin{aligned}
\left(\left[x_{1}, x_{2}\right]^{2}, x_{2}, x_{1} x_{3}\right)= & -\left(\left[x_{1}, x_{2}\right]^{2}, x_{1}, x_{2} x_{3}\right)+\left(x_{2}, x_{3},\left[x_{1}, x_{2}\right]^{2}\right) x_{1} \\
& +\left(x_{1}, x_{3},\left[x_{1}, x_{2}\right]^{2}\right) x_{2}
\end{aligned}
$$

Portanto $I\left(x_{2}, x_{1} x_{3}\right) \subseteq I\left(x_{1}, x_{2} x_{3}\right)+\sum_{i<j} I\left(x_{i}, x_{j}\right)$. Desta forma, temos que $I(r, s) \subseteq \sum_{i<j} I\left(x_{i}, x_{j}\right)+I\left(x_{1}, x_{2} x_{3}\right)$. Mas, da equação (2.32), temos que $I\left(x_{i}, x_{j}\right)=I\left(x_{1}, x_{2} x_{3}\right)=(0)$ de onde segue que $I(r, s)=(0)$, e portanto, $\left[x_{1}, x_{2}\right]^{2} \in N$. Como o associador é linear em seus argumentos, temos que $N$ é uma subálgebra totalmente característica de $A$ (ver [12, p.289]). Daqui e pelo anterior, temos que $[x, y]^{2} \in N$, para quaisquer $x$ e $y \in \operatorname{Alt}\left[x_{1}, x_{2}, x_{3}\right]$. Assim (2.36) fica provada.

Seguindo com o mesmo esquema provemos que $\left[x_{1}, x_{2}\right] \circ\left[x_{3}, r\right] \in N$ para qualquer $r \in \operatorname{Alt}\left[x_{1}, x_{2}, x_{3}\right]$. Para isto, como no caso anterior, é suficiente provar que as seguintes equações são válidas:

$$
\begin{gathered}
\left(\left[x_{1}, x_{2}\right] \circ\left[x_{3}, r\right], x_{i}, x_{j}\right)=0 \\
\left(\left[x_{1}, x_{2}\right] \circ\left[x_{3}, r\right], x_{1}, x_{2} x_{3}\right)=0
\end{gathered}
$$


Linearizando (2.36) na variável y obtemos a seguinte identidade em $\operatorname{Alt}\left[X_{3}\right]$ :

$$
([x, y] \circ[x, z], r, s)=0
$$

Por outro lado, usando (2.8) e o teorema de Artin temos que:

$$
([x, y] \circ z, x, y)=[x, y] \circ(z, x, y)+z \circ([x, y], x, y)=0
$$

Provemos agora (2.38). Pela linearização parcial de (2.41) só é preciso considerar o caso em que $x_{i}=x_{3}$ ou $x_{j}=x_{3}$. É claro que basta considerar só um deles. Por exemplo $x_{i}=x_{3}$. Aplicando (2.23) e a linarização parcial de (2.24) temos $\left(\left[x_{1}, x_{2}\right] \circ\left[x_{3}, r\right], x_{3}, x_{j}\right)=-\left(\left[x_{1}, x_{2}\right] \circ\left[x_{3}, x_{j}\right], x_{3}, x_{r}\right)=0$, ficando assim provada a equação (2.40). Além do mais, aplicando novamente a linearização parcial de (2.41) e (2.40), temos:

$$
\begin{aligned}
\left(\left[x_{1}, x_{2}\right] \circ\left[x_{3}, r\right], x_{1}, x_{2} x_{3}\right)= & -\left(\left[x_{1}, x_{2} x_{3}\right] \circ\left[x_{3}, r\right], x_{1}, x_{2}\right) \\
= & \left(\left[x_{1}, x_{2} x_{3}\right] \circ\left[x_{1}, r\right], x_{3}, x_{2}\right)+\left(\left[x_{1}, x_{2} x_{3}\right] \circ\left[x_{3}, x_{2}\right], x_{1}, r\right) \\
& +\left(\left[x_{1}, x_{2} x_{3}\right] \circ\left[x_{1}, x_{2}\right], x_{3}, r\right) \\
= & \left(\left[x_{1}, x_{2} x_{3}\right] \circ\left[x_{3}, x_{2}\right], x_{1}, r\right)
\end{aligned}
$$

Observemos que em toda álgebra é satisfeita a seguinte relação:

$$
[x, y z]+[y, z x]+[z, x y]=-(x, y, z)-(y, z, x)-(z, x, y)
$$

Daqui, aplicando (2.3) e (2.40) temos que

$$
\begin{aligned}
{\left[x_{1}, x_{2} x_{3}\right]=} & -\left[x_{2}, x_{3} x_{1}\right] \circ\left[x_{3}, x_{2}\right]-\left[x_{3}, x_{1} x_{2}\right] \circ\left[x_{3}, x_{2}\right] \\
& -3\left(x_{1}, x_{2}, x_{3}\right) \circ\left[x_{3}, x_{2}\right] \\
= & {\left[x_{2}, x_{3} x_{1}\right] \circ\left[x_{2}, x_{3}\right]-\left[x_{3}, x_{1} x_{2}\right] \circ\left[x_{3}, x_{2}\right] \in N }
\end{aligned}
$$

Portanto (2.39) está provada. Assim $\left[x_{1}, x_{2}\right] \circ\left[x_{3}, r\right] \in N$ para qualquer $r \in A l t\left[X_{3}\right]$. Mas como o centro associativo é uma subálgebra completamente característica de $A$ temos que $[x, y] \circ[z, t] \in N$ para quaisquer $x, y, z, t \in$ $A l t\left[X_{3}\right]$ o que prova (2.37) e o Teorema 4.

Chamando $X_{3}$ o conjunto $x_{1}, x_{2}, x_{3}$, então, pelo teorema anterior, temos visto que para quaisquer $x, y, z, t \in \operatorname{Alt}\left[X_{3}\right]$ os elementos $[x, y]^{2} \mathrm{e}$ $[x, y] \circ[z, t] \in N\left(A l t\left[x_{1}, x_{2}, x_{3}\right]\right)$. A seguir, encontraremos alguns elementos do centro da álgebra alternativa livre com 3 geradores. 
Teorema 5 (Shestakov) Para quaisquer $x, y, z, r, s \in A l t\left[X_{3}\right]$, os elementos $(x, y, z)^{2}$ e $(x, y, z) \circ[r, s] \in Z\left(A l t\left[X_{3}\right]\right)$.

Demonstração: Como na demonstração do teorema anterior, para provar que $f=f\left(x_{1}, x_{2}, x_{3}\right) \in Z\left(A l t\left[X_{3}\right]\right)$, é suficiente provar que $\left[f, x_{i}\right]=$ $\left(f, x_{i}, x_{j}\right)=0$. Pelas equações $(2.33)$ e $(2.34)$ é claro que $\left(x_{1}, x_{2}, x_{3}\right)^{2} \in$ $Z\left(A l t\left[X_{3}\right]\right)$ e portanto, pelos argumentos já citados, o elemento $(x, y, z)^{2} \in$ $Z\left(A l t\left[X_{3}\right]\right)$.

Finalmente, para provar a segunda inclusão do teorema, é suficiente provar que $\left(x_{1}, x_{2}, x_{3}\right) \circ[r, s] \in Z\left(A l t\left[X_{3}\right]\right)$, para todo $r, s \in A l t\left[X_{3}\right]$. Podemos supor que $r$ e $s$ são monômios em $x_{1}, x_{2}, x_{3}$ e aplicar indução sobre $n=d(r)+d(s)$. Se $n=2$, o resultado segue de (2.3). Suponhamos que $n>2$. Como já foi observado, é suficiente provar que para os geradores $x_{i}$ são satisfeitas as seguintes identidades:

$$
\begin{gathered}
\left(\left(x_{1}, x_{2}, x_{3}\right) \circ[r, s], x_{i}, x_{j}\right)=0 \\
{\left[\left(x_{1}, x_{2}, x_{3}\right) \circ[r, s], x_{i}\right]=0}
\end{gathered}
$$

Comecemos provando (2.43). Se $d(r)>1$ e $d(s)>1$ então aplicando a linearização parcial de (2.41) e a hipótese de indução, temos que:

$$
\begin{aligned}
\left(\left(x_{1}, x_{2}, x_{3}\right) \circ[r, s], x_{i}, x_{j}\right)= & -\left(\left(x_{1}, x_{2}, x_{3}\right) \circ\left[x_{i}, s\right], r, x_{j}\right) \\
& -\left(\left(x_{1}, x_{2}, x_{3}\right) \circ\left[r, x_{j}\right], x_{i}, s\right) \\
& -\left(\left(x_{1}, x_{2}, x_{3}\right) \circ\left[x_{i}, x_{j}\right], r, s\right) \\
= & 0
\end{aligned}
$$

Caso contrário, podemos supor $r=x_{1}$ (analogamente $s=x_{1}$ ). Notemos que pela equação (2.3) e o teorema de Artin temos que

$$
\begin{aligned}
\left(\left(x_{1}, x_{2}, x_{3}\right) \circ\left[x_{i}, s\right], x_{i}, s\right)= & \left(x_{1}, x_{2}, x_{3}\right) \circ\left(\left[x_{i}, s\right], x_{i}, s\right) \\
& +\left[x_{i}, s\right] \circ\left(\left(x_{1}, x_{2}, x_{3}\right), x_{i}, s\right) \\
= & 0
\end{aligned}
$$

Linearizando esta equação e aplicando novamente (2.3) obtemos:

$$
\left(\left(x_{1}, x_{2}, x_{3}\right) \circ\left[x_{i}, s\right], x_{i}, x_{j}\right)=-\left(\left(x_{1}, x_{2}, x_{3}\right) \circ\left(\left[x_{i}, x_{j}\right], x_{i}, s\right)=0\right.
$$

Portanto, basta considerar o elemento $f=\left(\left(x_{1}, x_{2}, x_{3}\right) \circ\left[x_{1}, s\right], x_{2}, x_{3}\right)$, já que os outros são nulos. Usando a linearização parcial de (2.45), vemos que

$$
f \equiv-\left(\left(x_{1}, x_{2}, x_{3}\right) \circ\left[x_{2}, s\right], x_{1}, x_{3}\right)=-\left(\left(x_{1}, x_{2}, x_{3}\right) \circ\left[x_{3}, s\right], x_{2}, x_{1}\right)
$$


Por outro lado, da linearização parcial das identidades alternativas e aplicando indução, é fácil provar que $s$ pode ser escrito da seguinte forma: $s=\sum_{i=1}^{3} \alpha_{i}\left(x_{i} s_{i}\right)+\beta_{i}\left(s_{i}^{\prime} x_{i}\right)$, onde $\alpha_{i}, \beta_{i} \in \Phi$ e $s_{i}, s_{i}^{\prime}$ são monômios nas variáveis $x_{1}, x_{2}, x_{3}$ com $d\left(s_{i}\right)=d\left(s_{i}^{\prime}\right)=d(s)-1$. Desta forma, é suficiente considerar os elementos $f_{i}=\left(\left(x_{1}, x_{2}, x_{3}\right) \circ\left[x_{1}, x_{i} s_{i}\right], x_{2}, x_{3}\right)$ e $f_{i}^{\prime}=$ $\left(\left(x_{1}, x_{2}, x_{3}\right) \circ\left[x_{1}, s_{i}^{\prime} x_{i}\right], x_{2}, x_{3}\right)$. Além do mais, pela analogia da forma destes elementos, só provaremos a inclusão de um deles. Aplicando (2.46) se fosse necessário, podemos escrever $f_{i}= \pm\left(\left(x_{1}, x_{2}, x_{3}\right) \circ\left[x_{j}, x_{i} s_{i}\right], x_{i}, x_{k}\right)$, onde $\{i, j, k\}=\{1,2,3\}$ e $x_{j} \neq s_{i}$. Aplicando agora a equação (2.35), temos que:

$$
\begin{aligned}
\left(x_{1}, x_{2}, x_{3}\right) \circ\left[x_{j}, x_{i} s_{i}\right]= & \left(x_{1}, x_{2}, x_{3}\right) \circ\left[x_{i}, s_{i} x_{j}\right]-\left(x_{1}, x_{2}, x_{3}\right) \circ\left[s_{i}, x_{j} x_{i}\right] \\
& -3\left(x_{1}, x_{2}, x_{3}\right) \circ\left(x_{j}, x_{i}, s_{i}\right)
\end{aligned}
$$

Observemos que o último termo corresponde à linearização parcial da inclusão $\left(x_{1}, x_{2}, x_{3}\right)^{2} \in Z\left(A l t\left[X_{3}\right]\right)$, com respeito à variável $z$. Além do mais, pela equação (2.45), o elemento $\left(\left(x_{1}, x_{2}, x_{3}\right) \circ\left[x_{i}, s_{i} x_{j}\right], x_{i}, x_{k}\right)=0$. Se $d\left(s_{i}\right)>1$, repetimos o processo diminuindo novamente o grau de $s_{i}$ até chegar a $d\left(s_{i}\right)=1$ e, nesse caso, como $s_{i} \neq x_{j}$ temos que $s_{i} \in\left\{x_{i}, x_{k}\right\}$, portanto, aplicando novamente (2.45), temos que $\left(\left(x_{1}, x_{2}, x_{3}\right) \circ\left[s_{i}, x_{j} x_{i}\right], x_{i}, x_{j}\right)=0$. Portanto, $f_{i}=0$ para $i=1,2,3$. Desta forma temos provado que $f=0$, ficando provada a relação (126). Por último, aplicando a linearização parcial de (1.4) e (2.15) temos que:

$$
\begin{aligned}
{\left[\left(x_{1}, x_{2}, x_{3}\right) \circ[r, s], x_{1}\right]=} & \left(x_{1}, x_{2}, x_{3}\right) \circ\left[[r, s], x_{1}\right]+[r, s] \circ\left[\left(x_{1}, x_{2}, x_{3}\right), x_{1}\right] \\
= & \left(x_{1}, x_{2}, x_{3}\right) \circ\left[[r, s], x_{1}\right]+[r, s] \circ\left(x_{1}, x_{2},\left[x_{1}, x_{3}\right]\right) \\
= & \left(x_{1}, x_{2}, x_{3}\right) \circ\left[[r, s], x_{1}\right]+\left(x_{1}, x_{2},[r, s] \circ\left[x_{1}, x_{3}\right]\right) \\
& -\left[x_{1}, x_{3}\right] \circ\left(x_{1}, x_{2},[r, s]\right) \\
= & \left(x_{1}, x_{2}, x_{3}\right) \circ\left[[r, s], x_{1}\right]+\left(x_{1}, x_{2},[r, s] \circ\left[x_{3}, x_{1}\right]\right) \\
= & 0
\end{aligned}
$$

o que prova (2.44) e o Teorema 5.

O teorema de Shestakov nos diz que se a álgebra alternativa tem 3 geradores então os elementos $\left(x_{1}, x_{2}, x_{3}\right)^{2}$ e $\left(x_{1}, x_{2}, x_{3}\right) \circ[r, s]$ pertencem ao centro da álgebra. Em particular, $\left(x_{1}, x_{2}, x_{3}\right)^{2} \in K\left(\right.$ Alt $\left.\left[X_{3}\right]\right)$, simplificando assim a forma encontrada no caso da álgebra alternativa livre $A l t[X]$ (Teorema 3). 


\section{Capítulo 3}

\section{O problema de Shirshov}

\subsection{Algumas relações em Alt $r$}

Dada uma variedade $M$ de álgebras sobre um anel $\Phi$, denotaremos por $\mathbf{M}_{r}(\Phi)$ à sub-variedade gerada, pelo processo de Birkhoff, a partir da álgebra livre de posto $r$ em M. Nestas condições, obtemos a seguinte seqüência de subvariedades:

$$
\mathbf{M}_{1}(\Phi) \subseteq M_{2}(\Phi) \subseteq \cdots \subseteq \mathbf{M}_{r}(\Phi) \subseteq \cdots \subseteq \mathbf{M}
$$

Chamaremos de "posto base" de $\mathbf{M}$ ao menor $r$ que satisfaz $\mathbf{M}_{r}(\Phi)=\mathbf{M}$ e o denotaremos por $r_{b}(\mathbf{M})$.

Neste capítulo, consideraremos a variedade $A l t$ das álgebras alternativas e demonstraremos que, sob algumas hipóteses sobre o anel base, existe uma inclusão estrita entre as sub-variedades geradas pelo processo de Birkhoff a partir das álgebras alternativas livres de posto $r$ e $2^{r}+1$, para qualquer natural $r$, ou seja, $\operatorname{Alt}_{r}(\Phi) \subset \operatorname{Alt}_{2^{r}+1}(\Phi)$ e, portanto, $r_{b}(\operatorname{Alt}(\Phi))=\aleph_{0}$.

Para cada $r \in \mathbb{N}$ e cada partição de $r$ como soma de $k$ naturais, $r=$ $r_{1}+\cdots+r_{k}$, chamemos de $S_{r_{j}}$ ao subgrupo de $S_{r}$ que opera sobre $\left\{r_{1}+\cdots+\right.$ $\left.r_{j-1}+1, \ldots, r_{1}+\cdots r_{j-1}+r_{j}\right\}$ e fixa os outros elementos. Como os $S_{r_{j}}$ são disjuntos comutam entre si, de onde segue que $S_{r_{1}, \ldots, r_{k}}^{r}:=S_{r_{1}} \cdots S_{r_{k}}$ é um subgrupo de $S_{r}$. Se chamarmos de $V_{r_{1}, \ldots, r_{k}}^{r}$ a um sistema de representantes das classes laterais pela esquerda, então temos o seguinte resultado:

Lema 12 Se $r=k+l+m$, podemos escolher como sistema de representantes $V_{k, l, m}^{r}$ os conjuntos: $V_{k, l+m}^{r} V_{l, m}^{l+m}$ e $V_{k+l, m}^{r} V_{k, l}^{k+l}$. 
Demonstração: Pela definição de conjunto de representantes, temos que

$$
S_{r}=\bigcup_{\sigma \in V_{k, l+m}^{r}} \sigma S_{k} S_{l+m}
$$

Mas, do mesmo modo,

$$
S_{l+m}=\bigcup_{\tau \in V_{l, m}^{l+m}} \tau S_{l} S_{m}
$$

Portanto,

$$
S_{r}=\bigcup_{\substack{\sigma \in V_{k, l}^{r} \\ \text { r.m } \\ \tau \in V_{l, m}^{l+m}}}(\sigma \tau) S_{k} S_{l} S_{m} .
$$

Assim, $V_{k, l+m}^{r} V_{l, m}^{l+m}$ contém todos os representantes das classes de $S^{r}$ módulo $S_{k+l+m}^{r}$. Além do mais, temos que

$$
\left|V_{k, l+m}^{r} V_{l, m}^{l+m}\right|=\frac{r !}{k !(l+m) !} \frac{(l+m) !}{l ! m !}=\frac{r !}{k ! l ! m !}=\left|V_{k, l, m}^{r}\right|,
$$

portanto, $V_{k, l+m}^{r} V_{l, m}^{l+m}$ não contém elementos da mesma classe, sendo assim, um sistema de representantes. Analogamente, prova-se que $V_{k+l, m}^{r} V_{k, l}^{k+l}$ também é um sistema de representantes de $S_{r}$ módulo $S_{k, l, m}^{r}$.

A seguir, definamos indutivamente uma família de funções sobre uma álgebra alternativa qualquer. Para isto, coloquemos $f_{0}\left(x_{1}\right):=x_{1} ; f_{1}\left(x_{1}, x_{2}, x_{3}\right)$ $:=\left(x_{1}, x_{2}, x_{3}\right)$, e uma vez definidas as primeiras $r-1$ funções $f_{1}, \ldots, f_{r-1}$ definamos

$$
f_{r}\left(x_{1}, \ldots, x_{2 r+1}\right)=\sum_{\sigma \in V_{2 r-2,3}^{2 r+1}}(\operatorname{Sgn} \sigma) f_{r-1}\left(x_{1}, \ldots, x_{2 r-2},\left(x_{2 r-1}, x_{2 r}, x_{2 r+1}\right)\right)^{\sigma},
$$

onde usamos a notação $\left(a_{1}, \ldots, a_{n}\right)^{\sigma}:=\left(a_{\sigma(1)}, \ldots, a_{\sigma(n)}\right)$.

É claro que as funções assim definidas são antissimétricas em seus argumentos. Notemos também que se $\sigma$ e $\widetilde{\sigma}$ pertencem à mesma classe, então $\sigma=$ $\tilde{\sigma} \alpha, \operatorname{com} \alpha \in S_{2 r-3,3}$. Se $\alpha$ for par, então $f_{r-1}\left(x_{1}, \ldots, x_{2 r-2},\left(x_{2 r-1}, x_{2 r}, x_{2 r+1}\right)\right)^{\sigma}$ $=f_{r-1}\left(x_{1}, \ldots, x_{2 r-2},\left(x_{2 r-1}, x_{2 r}, x_{2 r+1}\right)\right)^{\tilde{\sigma}}$. Se $\alpha$ for ímpar, aparece um sinal (-) em cada lado da igualdade e, em qualquer dos casos, verifica-se que $(\operatorname{Sgn} \sigma) f_{r-1}\left(x_{1}, \ldots, x_{2 r-2},\left(x_{2 r-1}, x_{2 r}, x_{2 r+1}\right)\right)^{\sigma}=$

$$
(\operatorname{Sgn} \tilde{\sigma}) f_{r-1}\left(x_{1}, \ldots, x_{2 r-2},\left(x_{2 r-1}, x_{2 r}, x_{2 r+1}\right)\right)^{\tilde{\sigma}} .
$$

Portanto, a soma que define $f_{r}$ não depende da escolha dos representantes. 
Lema 13 Os $f_{r}$ definidos anteriormente satisfazem:

$$
\begin{aligned}
f_{r}\left(x_{1}, \ldots, x_{2 r}, x\right)= & \sum_{\sigma \in V_{2 r-2,2}^{2 r}}(\operatorname{Sgn} \sigma) f_{r-1}\left(x_{1}, \ldots, x_{2 r-2},\left(x_{2 r-1}, x_{2 r}, x\right)\right)^{\sigma} \\
& -\sum_{\sigma \in V_{2 r-3,3}^{2 r}}(\operatorname{Sgn} \sigma) f_{r-1}\left(x_{1}, \ldots, x_{2 r-3}, x,\left(x_{2 r-2}, x_{2 r-1}, x_{2 r}\right)\right)^{\sigma} .
\end{aligned}
$$

Demonstração: Na definição de $f_{r}\left(x_{1}, \ldots, x_{2 r+1}\right)$, fixemos $x_{2 r+1}=x$ e escrevamos $V_{2 r-2,3}^{2 r+1}=U_{2 r-2,3}^{2 r+1} \cup W_{2 r-2,3}^{2 r+1}$, onde

$$
\begin{aligned}
U_{2 r-2,3}^{2 r+1} & =\left\{\sigma \in V_{2 r-3,3}^{2 r+1} \text { tais que } 2 r+1 \in\{\sigma(k): k \geq 2 r-1\}\right\} \text { e } \\
W_{2 r-2,3}^{2 r+1} & =\left\{\sigma \in V_{2 r-3,3}^{2 r+1} \text { tais que } 2 r+1 \in\{\sigma(k): k<2 r-1\}\right\} .
\end{aligned}
$$

Notemos que se $\sigma \in U_{2 r-2,3}^{2 r+1}$, podemos supor que $\sigma(2 r+1)=2 r+1$, já que, de não ser assim, é suficiente multiplicar $\sigma$ por um elemento de $S_{2 r-2,3}$ para que aconteça, o que não muda o valor da soma. Do mesmo modo, podemos supor que se $\sigma \in W_{2 r-2,3}^{2 r+1}$, então $\sigma(2 r-2)=2 r+1$.

É claro que:

$\sum_{\sigma \in U_{2 r-2,3}^{2 r+1}}(\operatorname{Sgn} \sigma) f_{r-1}\left(x_{1}, \ldots, x_{2 r-2},\left(x_{2 r-1}, x_{2 r}, x\right)\right)^{\sigma}=$

$$
\sum_{\sigma \in V_{2 r-2,2}^{2 r}}(\operatorname{Sgn} \sigma) f_{r-1}\left(x_{1}, \ldots, x_{2 r-2},\left(x_{2 r-1}, x_{2 r}, x\right)\right)^{\sigma}
$$

pois para estes $\sigma, \sigma(2 r+1)=2 r+1$ está fixado. Vamos mostrar agora que

$$
\begin{aligned}
\sum_{\sigma \in W_{2 r-2,3}^{2 r+1}}(\operatorname{Sgn} \sigma) f_{r-1}\left(x_{1}, \ldots, x_{2 r-3}, x,\left(x_{2 r-1}, x_{2 r}, x_{2 r+1}\right)\right)^{\sigma}= \\
-\sum_{\tau \in V_{2 r-3,3}^{2 r}}(\operatorname{Sgn} \tau) f_{r-1}\left(x_{1}, \ldots, x_{2 r-3}, x,\left(x_{2 r-2}, x_{2 r-1}, x_{2 r}\right)\right)^{\tau} .
\end{aligned}
$$

Para isto, é suficiente provar que todo $\sigma \in W_{2 r-2,3}^{2 r+1}$ exista $\tau \in V_{2 r-3,3}^{2 r}$ tal que

$$
\begin{aligned}
(\operatorname{Sgn} \sigma) f_{r-1}\left(x_{1}, \ldots, x_{2 r-3},\right. & \left.x,\left(x_{2 r-1}, x_{2 r}, x_{2 r+1}\right)\right)^{\sigma}= \\
& -(\operatorname{Sgn} \tau) f_{r-1}\left(x_{1}, \ldots, x_{2 r-3}, x,\left(x_{2 r-2}, x_{2 r-1}, x_{2 r}\right)\right)^{\tau}
\end{aligned}
$$

já que ambos os conjuntos $W_{2 r-2,3}^{2 r+1}$ e $V_{2 r-3,3}^{2 r}$ têm a mesma quantidade de elementos. Seja $\sigma \in W_{2 r-2,3}^{2 r+1}$ arbitrária, definimos $\tau$ do seguinte modo:

$$
\tau(i)= \begin{cases}\sigma(i), & \text { se } 1 \leq i \leq 2 r-3 \\ \sigma(i+1), & \text { se } 2 r-3<i \leq 2 r\end{cases}
$$


É claro que $\tau$ assim definida pertence a $V_{2 r-3,3}^{2 r}$ e, além disso, $\tau=\sigma \alpha$, onde $\alpha=\left(\begin{array}{lll}2 r-2 & 2 r-1\end{array}\right)\left(\begin{array}{ll}2 r-1 & 2 r\end{array}\right)\left(\begin{array}{ll}2 r & 2 r+1\end{array}\right)$, de onde segue que $-\operatorname{Sgn} \tau=\operatorname{Sgn} \sigma$ e portanto a igualdade das somas se verifica, ficando provado o Lema 13.

Lema 14 As funções $f_{r}$, anteriormente definidas, satisfazem

$$
\begin{aligned}
& f_{r}\left(x_{1}, \ldots, x_{2 r}, x^{2}\right)= \\
& \quad \sum_{k=0}^{r} c_{r}^{k} \sum_{\sigma \in V_{2 k, 2 r-2 k}^{2 r}}(\operatorname{Sgn} \sigma) f_{k}\left(x_{1}, \ldots, x_{2 k}, x\right)^{\sigma} f_{r-k}\left(x_{2 k+1}, \ldots, x_{2 r}, x\right)^{\sigma}
\end{aligned}
$$

onde $c_{r}^{k}=\frac{r !}{k !(r-k) !}$ é o número binomial.

Demonstração: A demonstração segue por indução sobre $r$. Se $r=0$, $f_{0}\left(x^{2}\right)=f_{0}(x) f_{0}(x)=x^{2}$. Se $r=1, c_{1}^{0} f_{0}(x) f_{1}\left(x_{1}, x_{2}, x\right)+c_{1}^{1} f_{1}\left(x_{1}, x_{2}, x\right) f_{0}(x)=$ $f_{0}(x) \circ f_{1}\left(x_{1}, x_{2}, x\right)=x \circ\left(x_{1}, x_{2}, x\right)=\left(x_{1}, x_{2}, x^{2}\right)=f_{1}\left(x_{1}, x_{2}, x^{2}\right)$, portanto, para $r=1$ o lema é válido. Suponhamos agora que o resultado é válido para $r-1$, isto é,

$$
\begin{aligned}
& f_{r-1}\left(x_{1}, \ldots, x_{2 r-2}, x^{2}\right)= \\
& \quad \sum_{k=0}^{r-1} c_{r-1}^{k} \sum_{\sigma}(\operatorname{Sgn} \sigma) f_{k}\left(x_{1}, \ldots, x_{2 k}, x\right)^{\sigma} f_{r-1-k}\left(x_{2 k+1}, \ldots, x_{2 r-2}, x\right)^{\sigma}
\end{aligned}
$$

onde $\sigma \in V_{2 k, 2 r-2 k-2}^{2 r-2}$. Linearizando esta igualdade com respeito a $x$, obtemos $f_{r-1}\left(x_{1}, \ldots, x_{2 r-2}, x \circ y\right)=$

$$
\sum_{k=0}^{r-1} c_{r-1}^{k} \sum_{\sigma \in V_{2 k, 2 r-2 k-2}^{2 r-2}}(\operatorname{Sgn} \sigma)\left[g_{r-1}^{k}(x, y, \sigma)+g_{r-1}^{k}(y, x, \sigma)\right],
$$

onde $g_{r-1}^{k}(x, y, \sigma)=f_{k}\left(x_{1}, \ldots, x_{2 k}, x\right)^{\sigma} f_{r-1-k}\left(x_{2 k+1}, \ldots, x_{2 r-2}, y\right)^{\sigma}$. Agora, pelo Lema 13,

$$
\begin{aligned}
f_{r}\left(x_{1}, \ldots, x_{2 r}, x^{2}\right)= & \sum_{\sigma \in V_{2 r-2,2}^{2 r}}(\operatorname{Sgn} \sigma) f_{r-1}\left(x_{1}, \ldots, x_{2 r-2},\left(x_{2 r-1}, x_{2 r}, x^{2}\right)\right) \\
& -\sum_{\sigma \in V_{2 r-3,3}^{2 r}}(\operatorname{Sgn} \sigma) f_{r-1}\left(x_{1}, \ldots, x_{2 r-3}, x^{2},\left(x_{2 r-2}, x_{2 r-1}, x_{2 r}\right)\right)^{\sigma} \\
= & S_{1}-S_{2} .
\end{aligned}
$$

Usando (1.4) e (3.3) vemos que 


$$
\begin{aligned}
S_{1} & =\sum_{\sigma \in V_{2 r-2,2}^{2 r}}(\operatorname{Sgn} \sigma) f_{r-1}\left(x_{1}, \ldots, x_{2 r-2}, x \circ\left(x_{2 r-1}, x_{2 r}, x\right)\right)^{\sigma} \\
& =\sum_{\sigma \in V_{2 r-2,2}^{2 r}}(\operatorname{Sgn} \sigma) \sum_{k=0}^{r-1} c_{r-1}^{k} \sum_{\sigma_{1}}\left(\operatorname{Sgn} \sigma_{1}\right)\left[g_{r-1}^{k}\left(x, y(\sigma), \sigma \sigma_{1}\right)+g_{r-1}^{k}\left(y(\sigma), x, \sigma \sigma_{1}\right)\right]
\end{aligned}
$$

onde $\sigma_{1} \in V_{2 k, 2 r-2 k-2}^{2 r-2}$ e $y(\sigma):=\left(x_{2 r-1}, x_{2 r}, x\right)^{\sigma}$. Notemos que como $\sigma_{1} \in$ $V_{2 k, 2 r-2 k-2}^{2 r-2}, y(\sigma)=y\left(\sigma \sigma_{1}\right)$. Mudando a ordem das somas temos

$S_{1}=$

$$
\sum_{k=0}^{r-1} c_{r-1}^{k}\left\{\sum_{\sigma \in V_{2 r-2,2}^{2 r}} \sum_{\sigma_{1}}\left(\operatorname{Sgn}\left(\sigma \sigma_{1}\right)\right)\left[g_{r-1}^{k}\left(x, y\left(\sigma \sigma_{1}\right), \sigma \sigma_{1}\right)+g_{r-1}^{k}\left(y\left(\sigma \sigma_{1}\right), x, \sigma \sigma_{1}\right)\right]\right\}
$$

onde $\sigma_{1} \in V_{2 k, 2 r-2 k-2}^{2 r-2}$. Se agora aplicamos o Lema 12 obtemos:

$$
S_{1}=\sum_{k=0}^{r-1} c_{r-1}^{k} \sum_{\sigma \in V_{2 k, 2 r-2 k-2,2}^{2 r}}(\operatorname{Sgn} \sigma)\left[g_{r-1}^{k}(x, y(\sigma), \sigma)+g_{r-1}^{k}(y(\sigma), x, \sigma)\right] .
$$

Por outro lado, pela antisimetria de $f_{r-1}$, temos

$$
-S_{2}=\sum_{\sigma \in V_{2 r-3,3}^{2 r}}(\operatorname{Sgn} \sigma) f_{r-1}\left(x_{1}, \ldots, x_{2 r-3}, z(\sigma), x^{2}\right)
$$

onde $z(\sigma)=\left(x_{2 r-2}, x_{2 r-1}, x_{2 r}\right)^{\sigma}$. Agora, pela hipótese de indução, $f_{r-1}\left(y_{1}, \ldots, y_{2 r-2}, x^{2}\right)=$

$$
\sum_{k=0}^{r-1} c_{r-1}^{k} \sum_{\sigma \in V_{2 k, 2 r-2 k-2}^{2 r-2}}(\operatorname{Sgn} \sigma) f_{k}\left(y_{1}, \ldots, y_{2 k}, x\right)^{\sigma} f_{r-1-k}\left(y_{2 k+1}, \ldots, y_{2 r-2}, x\right)^{\sigma}
$$

e pelo mesmo argumento usado na demonstração do Lema 13, se substituir$\operatorname{mos} y_{1}=x_{1}, \ldots, y_{2 r-3}=x_{2 r-3}$ e $y_{2 r-2}=\left(x_{2 r-2}, x_{2 r-1}, x_{2 r}\right)=z$, temos que $f_{r-1}\left(x_{1}, \ldots, x_{2 r-3}, z, x^{2}\right)=$

$$
\begin{aligned}
& \sum_{k=0}^{r-1} c_{r-1}^{k}\left\{\sum_{\sigma \in V_{2 k, 2 r-2 k-3}^{2 r-3}}(\operatorname{Sgn} \sigma) f_{k}\left(x_{1}, \ldots, x_{2 k}, x\right)^{\sigma} f_{r-1-k}\left(x_{2 k+1}, \ldots, x_{2 r-3}, z(\sigma), x\right)^{\sigma}\right. \\
& \left.\quad+\sum_{\sigma \in V_{2 k-1,2 r-2 k-2}^{2 r-3}}(\operatorname{Sgn} \sigma) f\left(x_{1}, \ldots, x_{2 k-1}, z(\sigma), x\right)^{\sigma} f_{r-1-k}\left(x_{2 k}, \ldots, x_{2 r-3}, x\right)^{\sigma}\right\} .
\end{aligned}
$$

Daqui, usando (3.4), concluímos 


$$
\begin{array}{r}
-S_{2}=\sum_{\sigma \in V_{2 r-3,3}^{2 r}}(\operatorname{Sgn} \sigma)\left\{\sum _ { k = 0 } ^ { r - 1 } c _ { r - 1 } ^ { k } \left[\sum_{\sigma_{1} \in V_{2 k, 2 r-2 k-3}^{2 r-3}}\left(\operatorname{Sgn} \sigma_{1}\right) h_{r-1}^{k}\left(x, z(\sigma), \sigma \sigma_{1}\right)\right.\right. \\
\left.\left.+\sum_{\sigma_{1} \in V_{2 k-1,2 r-2 k-2}^{2 r-3}}\left(\operatorname{Sgn} \sigma_{1}\right) t_{r-1}^{k}\left(z(\sigma), x, \sigma \sigma_{1}\right)\right]\right\},
\end{array}
$$

onde

$$
h_{r-1}^{k}(x, z, \sigma)=f_{k}\left(x_{1}, \ldots, x_{2 k}, x\right)^{\sigma} f_{r-1-k}\left(x_{2 k+1}, \ldots, x_{2 r-3}, z, x\right)^{\sigma}
$$

$\mathrm{e}$

$$
t_{r-1}^{k}(z, x, \sigma)=f_{k}\left(x_{1}, \ldots, x_{2 k-1}, z, x\right)^{\sigma} f_{r-1-k}\left(x_{2 k}, \ldots, x_{2 r-3}, x\right)^{\sigma} .
$$

Mudando a ordem das somas e usando o Lema 12, temos

$$
-S_{2}=\sum_{k=0}^{r-1} c_{r-1}^{k}\left\{\sum_{\sigma \in V_{2 k, 2 r-2 k-3,3}^{2 r}}(\operatorname{Sgn} \sigma) h_{r-1}^{k}(x, z(\sigma), \sigma)+\sum_{\sigma_{1}}(\operatorname{Sgn} \sigma) t_{r-1}^{k}(z(\sigma), x, \sigma)\right\},
$$

onde $\sigma_{1} \in V_{2 k-1,2 r-2 k-3,3}^{2 r}$. Portanto, depois de reagrupar as somas, $S_{1}-S_{2}$

$$
\begin{aligned}
= & \sum_{k=0}^{r-1} c_{r-1}^{k}\left\{\sum_{\sigma \in V_{2 k, 2 r-2 k-2,2}^{2 r}}(\operatorname{Sgn} \sigma) g_{r-1}^{k}(x, y(\sigma), \sigma)+\sum_{\sigma \in V_{2 k, 2 r-2 k-3,3}^{2 r}}(\operatorname{Sgn} \sigma) h_{r-1}^{k}(x, z(\sigma), \sigma)\right\} \\
& +\sum_{k=0}^{r-1} c_{r-1}^{k}\left\{\sum_{\sigma \in V_{2 k, 2 r-2 k-2}^{2 r}}(\operatorname{Sgn} \sigma) g_{r-1}^{k}(y(\sigma), x, \sigma)+\sum_{\sigma \in V_{2 k-1,2 r-2 k-2,3}^{2 r}}(\operatorname{Sgn} \sigma) t_{r-1}^{k}(z(\sigma), x, \sigma)\right\} \\
= & \sum_{k=0}^{r-1} c_{r-1}^{k} F_{k}+\sum_{k=0}^{r-1} c_{r-1}^{k} E_{k}
\end{aligned}
$$

Agora, manipulando as somas e usando o Lema 12, tentaremos mudar adequadamente a aparência destas.

$$
\begin{aligned}
F_{k}= & \sum_{\sigma \in V_{2 k, 2 r-2 k}^{2 r}} \sum_{\sigma_{1} \in V_{2 r-2 k-2,2}^{2 r}}(\operatorname{Sgn} \sigma)\left(\operatorname{Sgn} \sigma_{1}\right) g_{r-1}^{k}\left(x, y\left(\sigma \sigma_{1}\right), \sigma \sigma_{1}\right) \\
& +\sum_{\sigma \in V_{2 k, 2 r-2 k}^{2 r}} \sum_{\sigma_{1} \in V_{2 r-2 k-3,3}^{2 r-2 k}}(\operatorname{Sgn} \sigma)\left(\operatorname{Sgn} \sigma_{1}\right) h_{r-1}^{k}\left(x, z\left(\sigma \sigma_{1}\right), \sigma \sigma_{1}\right) .
\end{aligned}
$$


Segue, aplicando o Lema 13, que

$$
\left.E_{k}=\sum_{\sigma \in V_{2 k, 2 r-2 k-2}^{2 r}}(\operatorname{Sgn} \sigma) f_{k+1}\left(x_{1}, \ldots, x_{2 k+2}, x\right)\right)^{\sigma} f_{r-1-k}\left(x_{2 k+3}, \ldots, x_{2 r}, x\right)^{\sigma} .
$$

Finalmente,

$$
\begin{aligned}
f_{r}( & \left.x_{1}, \ldots, x_{2 r}, x^{2}\right) \\
= & \sum_{k=0}^{r-1} c_{r-1}^{k} F_{k}+\sum_{k=0}^{r-1} c_{r-1}^{k} E_{k} \\
= & \sum_{k=0}^{r-1} c_{r-1}^{k} \sum_{\sigma \in V_{2 k, 2 r-2 k}^{2 r}}(\operatorname{Sgn} \sigma) f_{k}\left(x_{1}, \ldots, x_{2 k}, x\right)^{\sigma} f_{r-k}\left(x_{2 k+1}, \ldots, x_{2 r}, x\right)^{\sigma} \\
= & \sum_{k=0}^{r-1} c_{r-1}^{k} \sum_{\sigma \in V_{2 k+2,2 r-2 k-2}^{2 r}}(\operatorname{Sgn} \sigma) f_{k+1}\left(x_{1}, \ldots, x_{2 k+2}, x\right)^{\sigma} f_{r-k-1}\left(x_{2 k+3}, \ldots, x_{2 r}, x\right)^{\sigma} \\
= & \sum_{k=1}^{r-1}\left(c_{r-1}^{k}+c_{r-1}^{k-1}\right) \sum_{\sigma \in V_{2 k, 2 r-2 k}^{2 r}}(\operatorname{Sgn} \sigma) f_{k}\left(x_{1}, \ldots, x_{2 k}, x\right)^{\sigma} f_{r-k}\left(x_{2 k+1}, \ldots, x_{2 r}, x\right)^{\sigma} \\
& +f_{0}(x) f_{r}\left(x_{1}, \ldots, x_{2 r}, x\right)+f_{r}\left(x_{1}, \ldots, x_{2 r}, x\right) f_{0}(x) \\
= & \sum_{k=0}^{r} c_{r}^{k} \sum_{\sigma \in V_{2 k, 2 r-2 k}^{2 r}}(\operatorname{Sgn} \sigma) f_{k}\left(x_{1}, \ldots, x_{2 k}, x\right)^{\sigma} f_{r-k}\left(x_{2 k+1}, \ldots, x_{2 r}, x\right)^{\sigma} .
\end{aligned}
$$

Com o que acaba a demonstração.

Corolário 6 Em toda álgebra alternativa, a seguinte identidade é satifeita:

$$
f_{r}\left(x_{1}, \ldots, x_{2 r-1}, x^{2}, x\right) .
$$

Lema 15 As funções $f_{r}, r \geq 1$, definidas numa álgebra alternativa, satisfazem a seguinte relação:

$$
f_{r}\left(x_{1}, \ldots, x_{2 r-1}, x y x, y\right)=f_{r}\left(x_{1}, \ldots, x_{2 r-1}, x, y x y\right) .
$$

Demonstração: Primeiro provemos que em toda álgebra alternativa é satisfeita a seguinte igualdade

$$
(z, x y x, t)=x(z, x, t) x+\{(z, x, t) y x\}
$$


onde $\{r s t\}=r(s t)+t(s r)$. Com efeito, pela equação (1.4) temos que

$$
\begin{aligned}
(r, s, x y x) & =-\left(r, s, x^{2} y\right)+(r, s,(x y) \circ x) \\
& =\left(x^{2} y, s, r\right)+x \circ(r, s, x y)+x y \circ(r, s, x)
\end{aligned}
$$

Portanto

$$
\begin{aligned}
(r, s, x y x)= & f\left(x^{2}, y, s r\right)+y[x \circ(x, s, r)]+(y, s, r) x^{2} \\
& +x \circ(r, s, x y)+(x y) \circ(r, s, x)
\end{aligned}
$$

Por outro lado, pelo Lema 3, (1.4) e sua linearização parcial temos

$$
\begin{aligned}
f\left(x^{2}, y, s, r\right)= & \left(\left[x^{2}, y\right], s, r\right)+\left(x^{2}, y,[s, r]\right) \\
= & (x \circ[x, y], s, r)+x \circ(x, y,[s, r]) \\
= & x \circ([x, y], s, r)+[x, y] \circ(x, s, r)+x \circ(x, y,[s, r]) \\
= & x \circ f(x, y, s, r)+[x, y] \circ(x, s, r) \\
= & x \circ(x y, s, r)-x \circ(y(x, s, r))-x \circ((y, s, r) x) \\
& +[x, y] \circ(x, s, r)
\end{aligned}
$$

Substituindo esta igualdade na equação (3.8), (3.7) fica provada.

Procedamos agora por indução sobre $r$. Se $r=1$, o lema reduz-se a (2.5).

Suponhamos que o lema é válido para $r-1$, isto é, que $f_{r-1}\left(x_{1}, \ldots, x_{2 r-3}, x y x, y\right)$ $=f_{r-1}\left(x_{1}, \ldots, x_{2 r-3}, x, y x y\right)$. Linearizando esta equação com respeito a $y$, obtemos

$f_{r-1}\left(x_{1}, \ldots, x_{2 r-3}, x,\{z x y\}\right)=$

$$
f_{r-1}\left(x_{1}, \ldots, x_{2 r-3}, x y x, z\right)+f_{r-1}\left(x_{1}, \ldots, x_{2 r-3}, x z x, y\right) .
$$

Agora, pelo mesmo argumento usado na prova do Lema 13, temos que $f_{r}\left(x_{1}, \ldots, x_{2 r-1}, x y x, y\right)=$

$$
\begin{aligned}
& \sum_{\sigma \in V_{2 r-2,1}^{2 r-1}}(\operatorname{Sgn} \sigma) f_{r-1}\left(x_{1}, \ldots, x_{2 r-2},\left(x_{2 r-1}, x y x, y\right)\right)^{\sigma} \\
+ & \sum_{\sigma \in V_{2 r-3,2}^{2 r-1}}(\operatorname{Sgn} \sigma) f_{r-1}\left(x_{1}, \ldots, x_{2 r-3}, x y x,\left(x_{2 r-2}, x_{2 r-1}, y\right)\right)^{\sigma} \\
- & \sum_{\sigma \in V_{2 r-3,2}^{2 r-1}}(\operatorname{Sgn} \sigma) f_{r-1}\left(x_{1}, \ldots, x_{2 r-3}, y,\left(x_{2 r-2}, x_{2 r-1}, x y x\right)\right)^{\sigma} \\
+ & \sum_{\sigma \in V_{2 r-4,3}^{2 r-1}}(\operatorname{Sgn} \sigma) f_{r-1}\left(x_{1}, \ldots, x_{2 r-4}, x y x, y,\left(x_{2 r-3}, x_{2 r-2}, x_{2 r-1}\right)\right)^{\sigma}
\end{aligned}
$$




$$
=S_{1}(x, y)+S_{2}(x, y)-S_{3}(x, y)+S_{4}(x, y)
$$

Usando a hipótese de indução, (2.5) e a antissimetria de $f_{r-1}$, segue que

$$
\begin{aligned}
S_{1}(x, y)+S_{4}(x, y)= & \sum_{\sigma \in V_{2 r-2,3}^{2 r-1}}(\operatorname{Sgn} \sigma) f_{r-1}\left(x_{1}, \ldots, x_{2 r-2},\left(x_{2 r-1}, x, y x y\right)\right)^{\sigma} \\
& +\sum_{\sigma \in V_{2 r-4,3}^{2 r-1}}(\operatorname{Sgn} \sigma) f_{r-1}\left(x_{1}, \ldots, x_{2 r-4}, x, y x y,\left(x_{2 r-3}, x_{2 r-2}, x_{2 r-1}\right)\right)^{\sigma} \\
= & -S_{1}(y, x)-S_{4}(y, x) .
\end{aligned}
$$

Por outro lado, usando (3.9) e (3.7),

$$
\begin{aligned}
S_{2}(x, y)-S_{3}(x, y)= & \sum_{\sigma \in V_{2 r-3,2}^{2 r-1}}(\operatorname{Sgn} \sigma) f_{r-1}\left(x_{1}, \ldots, x_{2 r-3}, y, x,\left(x_{2 r-2}, x_{2 r-1}, y\right) x\right)^{\sigma} \\
& +\sum_{\sigma \in V_{2 r-3,2}^{2 r-1}}(\operatorname{Sgn} \sigma) f_{r-1}\left(x_{1}, \ldots, x_{2 r-3}, x,\left\{\left(x_{2 r-2}, x_{2 r-1}, y\right) x y\right\}\right)^{\sigma} \\
& \quad-\sum_{\sigma \in V_{2 r-3,2}^{2 r-1}}(\operatorname{Sgn} \sigma) f_{r-1}\left(x_{1}, \ldots, x_{2 r-3}, y, x\left(x_{2 r-2}, x_{2 r-1}, y\right) x\right)^{\sigma} \\
& \quad-\sum_{\sigma \in V_{2 r-3,2}^{2 r-1}}(\operatorname{Sgn} \sigma) f_{r-1}\left(x_{1}, \ldots, x_{2 r-3}, y,\left\{\left(x_{2 r-2}, x_{2 r-1}, x\right) y x\right\}\right)^{\sigma} \\
= & \sum_{\sigma \in V_{2 r-3,2}^{2 r-1}}(\operatorname{Sgn} \sigma) f_{r-1}\left(x_{1}, \ldots, x_{2 r-3}, x,\left\{\left(x_{2 r-2}, x_{2 r-1}, y\right) x y\right\}\right)^{\sigma} \\
& -\sum_{\sigma \in V_{2 r-3,2}^{2 r-1}}(\operatorname{Sgn} \sigma) f_{r-1}\left(x_{1}, \ldots, x_{2 r-3}, y,\left\{\left(x_{2 r-2}, x_{2 r-1}, x\right) y x\right\}\right)^{\sigma} \\
= & -S_{2}(y, x)+S_{3}(y, x) .
\end{aligned}
$$

Portanto,

$f_{r}\left(x_{1}, \ldots, x_{2 r-1}, x y x, y\right)=-f_{r}\left(x_{1}, \ldots, x_{2 r-1}, y x y, x\right)=f_{r}\left(x_{1}, \ldots, x_{2 r-1}, x, y x y\right)$,

ficando provado o lema.

\subsection{A inclusão $A l t_{m}(\Phi) \subset A l t_{2^{m}+1}(\Phi)$}

Nesta seção, se não especificarmos o contrário, denotaremos por $A$ à álgebra alternativa livre de dimensão $\aleph_{0}$ e por $A_{m}$ à de dimensão $m$. Escreveremos também $\operatorname{Alt}_{m}(\Phi)$ para enfatizar que a álgebra é considerada sobre o anel $\Phi$.

Para começar, provaremos três resultados básicos do quais vamos precisar. 
Lema 16 Seja $A$ uma álgebra alternativa e $P(A)$ o $\Phi$-módulo gerado pelos elementos da forma $x^{2}$ e xyx, com $x, y \in A$. Então $P(A)$ é um ideal em $A$.

Demonstração: Primeiro, notemos que se $a, b, c \in A$, então o elemento

$$
(a+b)^{2}-a^{2}-b^{2}=a \circ b \in P(A) .
$$

Além do mais, o elemento

$$
(a+c) b(a+c)-a b a-c b c=(a b) c+(c b) a=a(b c)+c(b a) \in P(A) .
$$

Observemos que os elementos $a \circ b$ e $a(b c)+(c b) a$ são, de algum modo, linearizações de $a^{2}$ e $a b a$. Agora estamos em condições de provar o lema.

Com efeito, $a^{2} b=(a b) \circ a-a b a \in P(A)$ e do mesmo modo, $b a^{2}=$ $(b a) \circ a-a b a \in P(A)$. Por outro lado, pela identidade esquerda de Moufang e a equação (3.11), temos que

$$
\begin{aligned}
(a b a) c=a[b(a c)] & =a[b(a c)]+(a c)(b a)-(a c)(b a) \\
& =a[b(a c)]+(a c)(b a)-a(c b) a \in P(A) .
\end{aligned}
$$

Finalmente, $c(a b a)=c \circ(a b a)-(a b a) c \in P(A)$. Portanto, como $a, b$ e $c$ são arbitrários, segue que $P(A)$ é um ideal de $A$.

Se chamarmos $\bar{A}=A / P(A)$, é fácil provar que $\bar{A}$ é anticomutativa e antiassociativa.

Dado um conjunto de símbolos $x_{1}, x_{2}, \ldots$ denotaremos por

$$
\left\langle x_{i_{1}}, \ldots, x_{i_{r}}\right\rangle:=\left\{\cdots\left[\left(x_{i_{1}} x_{i_{2}}\right) x_{i_{3}}\right] x_{i_{4}} \cdots\right\} x_{i_{r}}=x_{i_{1}} R_{x_{i_{2}}} \cdots R_{x_{i_{r}}},
$$

onde $R_{a}$ é o operador de multiplicação por a pela direita. Adotando esta notação, temos o seguinte resultado:

Lema 17 Se $B$ é uma álgebra anticomutativa e antiassociativa, com geradores $x_{1}, x_{2}, \ldots$ então, qualquer elemento $r \in B$ pode ser escrito como combinação linear de palavras $w$, onde $w=\left\langle x_{i_{1}}, \ldots, x_{i_{k}}\right\rangle$, com $i_{1}<\cdots<i_{k}$.

Demonstração: A demonstração segue por indução sobre $r=\mathrm{d}(v)$. Se $r=1$, então $v=\sum \alpha_{i} x_{i}$ e o lema é satisfeito. Suponhamos que é certo para $\mathrm{d}(v)<r$, basta prová-lo para o caso em que $v$ é uma palavra. Seja $v$ com $\mathrm{d}(v)=r$, podemos escrever $v=v_{1} v_{2}$, com $\mathrm{d}\left(v_{1}\right)<r$ e $\mathrm{d}\left(v_{2}\right)<r$, pela 
hipótese de indução, $v_{i}=\left\langle v_{i}\right\rangle, i=1$, 2. Escrevamos $v_{2}=\left\langle v_{2}^{\prime} x_{i}\right\rangle$, obtendo $\operatorname{assim} v=v_{1}\left(v_{2}^{\prime} x_{i}\right)=-v_{1}\left(x_{i} v_{2}^{\prime}\right)=\left(v_{1} x_{i}\right) v_{2}^{\prime}$. Continuando com este processo e mudando o sinal, se for necessário, podemos escrever $v_{1} x_{i}=\left\langle v_{1}^{\prime}\right\rangle$, reduzindo no final $d\left(v_{2}\right)$ a 1 , ou seja, chegando ao caso $v=\bar{v}_{1} x_{k}$, com $\bar{v}_{1}=\left\langle\bar{v}_{1}\right\rangle$. Desta forma, conseguimos escrever $v$ da seguinte forma:

$$
v=\left\{\left\{\cdots\left[\left(x_{i_{1}} x_{i_{2}}\right) x_{i_{3}}\right] \cdots\right\} x_{i_{1}}\right\} x_{k} .
$$

Agora, permutando de 2 em 2, usando a anticomutatividade e antiassociatividade de $B$, podemos escrever

$$
v= \pm\left\{\cdots\left\{\left[\left(x_{i_{1}} x_{i_{2}}\right) x_{i_{3}}\right] \cdots\right\} x_{k} \cdots\right\} x_{i_{l}}
$$

com $i_{1}<i_{2}<\cdots<i_{j} \leq k<i_{j+1}<\cdots i_{l}$.

Agora estamos em condições de provar o seguinte teorema:

Teorema 6 Na álgebra $A_{m}$, a seguinte identidade é satisfeita:

$$
f_{2^{m-1}}\left(x_{1}, \ldots, x_{2^{m}+1}\right)=0 \text {. }
$$

Demonstração: Linearizando (3.5), (3.6) e usando a antissimetria de $f_{r}$, obtemos as seguintes equações:

$$
\begin{aligned}
f_{r}\left(x_{1}, \ldots, x_{2 r-1}, x^{2}, y\right)= & f_{r}\left(x_{1}, \ldots, x_{2 r-1}, x, x \circ y\right) \\
f_{r}\left(x_{1}, \ldots, x_{2 r-1}, x y x, z\right)= & f_{r}\left(x_{1}, \ldots, x_{2 r-1}, y, x z x\right) \\
& +f_{r}\left(x_{1}, \ldots, x_{2 r-1}, x,\{y x z\}\right) .
\end{aligned}
$$

Se considerarmos $P\left(A_{m}\right)$, o $\phi$-submódulo de $A_{m}$ gerado pelos elementos da forma $x^{2}$ e $x y x$, com $x, y \in A_{m}$, então, pelos lemas anteriores, $P\left(A_{m}\right)$ é um ideal de $A_{m}$ e, módulo $P\left(A_{m}\right)$, todo elemento de $A_{m}$ escreve-se como combinação linear de elementos da forma

$$
u=x_{i_{1}} R_{x_{i_{2}}} \cdots R_{x_{i_{k}}},
$$

onde os $x_{i}$ são os geradores livres de $A_{m}$ e $i_{1}<\cdots<i_{k}$.

Tomemos agora $y_{1}, \ldots, y_{2^{m}+1} \in A_{m}$. Como os elementos de $P\left(A_{m}\right)$ são combinações lineares de elementos da forma $x^{2}$ e $x y x$, usando (3.12) e (3.13), podemos escrever (ver [1, p.261-262])

$$
f_{2^{m-1}}\left(y_{1}, \cdots, y_{2^{m}+1}\right)=\sum \alpha_{i} f_{2^{m-1}}\left(u_{i_{1}}, \ldots, u_{i_{2} m}, u\right),
$$


onde $u_{i_{k}}, u \in A_{m}$ e os $u_{i_{k}}$ têm a forma de (3.14). Mas em $A_{m}$ existem somente $2^{m}-1$ elementos não nulos com a forma descrita. Portanto, o conjunto $\left\{u_{i_{1}}, \ldots, u_{i_{2} m}\right\}$ é linearmente dependente, de onde segue, pela antissimetria de $f_{2^{m-1}}$ que

$$
f_{2^{m-1}}\left(y_{1}, \ldots, y_{2^{m}+1}\right)=0
$$

o que prova o teorema.

Lema 18 Toda álgebra alternativa solúvel de índice 2 satisfaz a seguinte relação:

$$
f_{r}\left(x_{1}, \ldots, x_{2 r+1}\right)=\varphi(r) \sum_{\sigma \in V_{3,2 r-2}^{2 r+1}}(\operatorname{Sgn} \sigma)\left[\left(x_{1}, x_{2}, x_{3}\right) R_{x_{4}} \cdots R_{x_{2 r+1}}\right]^{\sigma}
$$

onde $\varphi(r)=(r-1) !(2 r-3) !$.

Demonstração: Seja $A$ uma álgebra alternativa solúvel de índice 2 e $x_{1}, \ldots$, $x_{2 r+1} \in A$. Provemos o lema por indução sobre $r$. Se $r=1$, a afirmação é óbvia. Suponhamos que provamos que a relação é verdadeira para $r-1$, isto é, que

$$
f_{r-1}\left(x_{1}, \ldots, x_{2 r-1}\right)=\varphi(r-1) \sum_{\sigma \in V_{3,2 r-4}^{2 r-1}}(\operatorname{Sgn} \sigma)\left[\left(x_{1}, x_{2}, x_{3}\right) R_{x_{4}} \cdots R_{x_{2 r-1}}\right]^{\sigma} .
$$

Então, pela própria definição de $f_{r}$, temos que

$$
f_{r}\left(x_{1}, \ldots, x_{2 r+1}\right)=\sum_{\sigma \in V_{2 r-2,3}^{2 r+1}}(\operatorname{Sgn} \sigma) f_{r-1}\left(x_{1}, \ldots, x_{2 r-2},\left(x_{2 r-1}, x_{2 r}, x_{2 r+1}\right)\right)^{\sigma} .
$$

Usando agora o mesmo argumento da demonstração do Lema 13 e a hipótese de indução, obtemos

$$
\begin{aligned}
& f_{r}\left(x_{1}, \ldots, x_{2 r+1}\right)= \\
& \quad \varphi(r-1) \sum_{\sigma \in V_{2 r-2,3}^{2 r+1}}(\operatorname{Sgn} \sigma) \sum_{\sigma_{1} \in V_{3,2 r-5}^{2 r-2}}\left(\operatorname{Sgn} \sigma_{1}\right)\left[\left(x_{1}, x_{2}, x_{3}\right) R_{x_{4}} \cdots R_{x_{2 r-1}}\right]^{\sigma \sigma_{1}} R_{y(\sigma)} \\
& \quad+\varphi(r-1) \sum_{\sigma \in V_{2 r-2,3}^{2 r+1}}(\operatorname{Sgn} \sigma) \sum_{\sigma_{1} \in V_{2,2 r-4}^{2 r-2}}\left(\operatorname{Sgn} \sigma_{1}\right)\left[\left(y(\sigma), x_{1}, x_{2}\right) R_{x_{3}} \cdots R_{x_{2 r-2}}\right]^{\sigma \sigma_{1}}
\end{aligned}
$$

onde $y(\sigma)=y\left(\sigma \sigma_{1}\right)=\left(x_{\sigma(2 r-1)}, x_{\sigma(2 r)}, x_{\sigma(2 r+1)}\right)$. Notemos que, como $A$ é solúvel de índice 2, para todo $\sigma \in V_{2 r-2,3}^{2 r+1}$ e $\sigma_{1} \in V_{3,2 r-5}^{2 r-2}$,

$$
\begin{aligned}
{\left[\left(x_{1}, x_{2}, x_{3}\right) R_{x_{4}} \cdots R_{x_{2 r-2}}\right]^{\sigma \sigma_{1}} R_{y(\sigma)}=} & \left(\left[\left(x_{1}, x_{2}, x_{3}\right) R_{x_{4}} \cdots R_{x_{2 r-3}}\right]^{\sigma \sigma_{1}} x_{\sigma \sigma_{1}(2 r-2)}\right) \\
& \times\left(\left(x_{2 r-1}, x_{2 r}, x_{2 r-1}\right)^{\sigma}\right) \subset A^{2} A^{2}=(0)
\end{aligned}
$$


de onde segue que a primeira soma é zero. Consideremos agora a segunda soma. Pelo Lema 12 e observando que $\left(x_{1}, x_{2}, x_{3}\right)\left(x_{4}, x_{5}\right)^{\sigma}=0$ para todo $\sigma$ temos que

$$
\begin{aligned}
\sum_{\sigma \in V_{2 r-2,3}^{2 r+1}}(\operatorname{Sgn} \sigma) \sum_{\sigma_{1} \in V_{2,2 r-4}^{2 r}-2}\left(\operatorname{Sgn} \sigma_{1}\right) & {\left[\left(y\left(\sigma \sigma_{1}\right), x_{1}, x_{2}\right) R_{x_{3}} \cdots R_{x_{2 r-2}}\right]^{\sigma \sigma_{1}} } \\
= & \sum_{\sigma \in V_{2,2 r-4,3}^{2 r+1}}(\operatorname{Sgn} \sigma)\left[\left(y(\sigma), x_{1}, x_{2}\right) R_{x_{3}} \cdots R_{x_{2 r-2}}\right]^{\sigma}
\end{aligned}
$$

Aplicando a permutação $\left(\left(\begin{array}{ll}1 & 2 r+1\end{array}\right)\left(\begin{array}{ll}1 & 2 r\end{array}\right) \cdots\left(\begin{array}{ll}1 & 2\end{array}\right)\right)^{3}$ nesta soma obtemos:

$$
\begin{aligned}
\sum_{\sigma \in V_{2 r-2,3}^{2 r+1}}(\operatorname{Sgn} \sigma) \sum_{\sigma 1 \in V_{2,2 r-4}^{2 r-2}} & \left(\operatorname{Sgn} \sigma_{1}\right)\left[\left(y\left(\sigma \sigma_{1}\right), x_{1}, x_{2}\right) R_{x_{3}} \cdots R_{x_{2 r-2}}\right]^{\sigma \sigma_{1}} \\
= & \sum_{\sigma \in V_{3,2,2 r-4}^{2 r+1}}(\operatorname{Sgn} \sigma)\left(\left(\left(x_{1}, x_{2}, x_{3}\right)\right), x_{4}, x_{5}\right) R_{x_{6}} \cdots R_{x_{2 r+1}}^{\sigma} \\
= & \left.\sum_{\sigma \in V_{3,2,2 r-4}^{2 r+1}}(\operatorname{Sgn} \sigma)\left(\left(x_{1}, x_{2}, x_{3}\right)\right) R_{x_{4}}, R_{x_{5}} \cdots R_{x_{2 r+1}}\right) \sigma
\end{aligned}
$$

Por outro lado, dados $m, n \geq 4$, é fácil ver que, considerando $\alpha=$ $(4 m)(5 n)$, verifica-se que

$$
\left(x_{1}, x_{2}, x_{3}\right) R_{x_{4}} \cdots R_{x_{2 r+1}}=\left[\left(x_{1}, x_{2}, x_{3}\right) R_{x_{4}} \cdots R_{x_{2 r+1}}\right]^{\alpha} .
$$

Notemos que nem sempre $\operatorname{Sgn} \alpha=1$, basta pensar, por exemplo, no caso $n>5$ e $m=4$.

Se considerarmos agora $\sigma \in V_{3,2 r-2}^{2 r+1}$ e $\alpha=\left(\begin{array}{ll}4 & m\end{array}\right)(5 n)$ como acima, obtemos

$$
\begin{aligned}
(\operatorname{Sgn} \sigma)\left[\left(x_{1}, x_{2}, x_{3}\right) R_{x_{4}} \cdots R_{x_{2 r+1}}\right]^{\sigma} & =(\operatorname{Sgn} \sigma)(\operatorname{Sgn} \alpha)\left[\left(x_{1}, x_{2}, x_{3}\right) R_{x_{4}} \cdots R_{x_{2 r+1}}\right]^{\sigma \alpha} \\
& =(\operatorname{Sgn} \sigma \alpha)\left[\left(x_{1}, x_{2}, x_{3}\right) R_{x_{4}} \cdots R_{x_{2 r+1}}\right]^{\sigma \alpha}
\end{aligned}
$$

e se identificarmos os $\alpha$ 's da forma $(4 m)(5 n)$ com os da forma $(4 n)(5 m)$ e variamos $\sigma \in V_{3,2 r-2}^{2 r+1}$, obtemos exatamente os representantes de $V_{3,2,2 r-4}^{2 r+1}$. Além disso, o número de $\alpha$ existentes com essas características é $\left(\begin{array}{c}2 r-2 \\ 2\end{array}\right)=$ $\frac{(2 r-2) !}{2 !(2 r-4) !}$. Portanto,

$f_{r}\left(x_{1}, \ldots, x_{2 r+1}\right)=\varphi(r-1) \frac{(2 r-2) !}{2 !(2 r-4) !} \sum_{\sigma \in V_{3,2 r-2}^{2 r+1}}(\operatorname{Sgn} \sigma)\left[\left(x_{1}, x_{2}, x_{3}\right) R_{x_{4}} \cdots R_{x_{2 r+1}}\right]^{\sigma}$. 
Mas $\varphi(r-1) \frac{(2 r-2) !}{2 !(2 r-4) !}=(r-2) !(2 r-5) ! !(r-1)(2 r-3)=\varphi(r)$, ficando provado o lema.

Corolário 7 Suponhamos que $\Phi$ não contém elementos de ordem aditiva $\leq$ $2 r+1$. Então, na $\Phi$-álgebra alternativa livre $A_{2 r+1}$ com geradores $\left\{x_{1}, \ldots, x_{2 r+1}\right\}$, $o$ elemento $f\left(x_{1}, \ldots, x_{2 r+1}\right)$ é não nulo.

Demonstração: Nas condições do enunciado, se tal elemento for zero, então em toda $\Phi$-álgebra alternativa solúvel de índice $2, f_{r}\left(x_{1}, \ldots, x_{2 r+1}\right)=0$ é uma identidade. Mas, no exemplo de Dorofeev (ver [12, p.127]), substituindo $x_{1}=x, x_{2}=e_{2}, \ldots, x_{2 r+1}=e_{2 r+1}$, temos que para qualquer $\sigma \in S_{2 r+1}$,

$$
\left[\left(x_{1}, x_{2}, x_{3}\right) R_{x_{4}} \cdots R_{x_{2 r+1}}\right]^{\sigma}=(\operatorname{Sgn} \sigma)\left(x_{1}, x_{2}, x_{3}\right) R_{x_{4}} \cdots R_{x_{2 r+1}} .
$$

Mas pelas regras de multiplicação, $\left[\left(x_{1}, x_{2}, x_{3}\right) R_{x_{4}} \cdots R_{x_{2 r+1}}\right]^{\sigma}=0$ se e somente se $\sigma(k)=1$, para algum $k \geq 4$ o que acontece em $C_{2}^{2 r}$ dos casos, portanto,

$$
\begin{aligned}
f_{r}\left(x, e_{2}, \ldots, e_{2 r+1}\right) & =(r-1) !(2 r-3) ! ! \frac{(2 r) !}{(2 r-2) ! 2 !}\left(x, e_{2}, e_{2}\right) R_{e_{4}} \cdots R_{e_{2 r+1}} \\
& =r !(2 r-1) ! x R_{e_{2}} \cdots R_{e_{2 r+1}} \neq 0
\end{aligned}
$$

o que contradiz que $f_{r}\left(x_{1}, \ldots, x_{2 r+1}\right)=0$ seja uma identidade, ficando assim provado o corolário.

Teorema 7 (Shestakov) Suponhamos que $\Phi$ não contém elementos de ordem aditiva finitos. Então, para todo $m$ a inclusão $\operatorname{Alt}_{m}(\Phi) \subset \operatorname{Alt}_{2^{m}+1}(\Phi)$ é estrita.

Demonstração: Pelo Teorema $6, f_{2^{m-1}}\left(x_{1}, \ldots, x_{2^{m}+1}\right)=0$ é uma identidade em $A_{m}$ e, portanto, em $\operatorname{Alt}_{m}(\Phi)$. Por outro lado, colocando $r=2^{m-1}$ no corolário 7 , vemos que $f_{2^{m-1}}\left(x_{1}, \ldots, x_{2^{m}+1}\right)$ é diferente de zero em $A_{2\left(2^{m-1}\right)+1}=$ $A_{2^{m+1}}$, de onde segue que $\operatorname{Alt}_{m}(\Phi) \neq \operatorname{Alt}_{2^{m}+1}(\Phi)$.

Desta forma, vemos que sob as hipóteses do Teorema 7 a sequencia de variedades: $A l t_{1}(\Phi) \subseteq A l t_{2}(\Phi) \subseteq \ldots \subseteq A l t(\Phi)$ não se estabiliza e portanto o posto base $r_{b}(A l t(\Phi))=\aleph_{0}$. 


\section{Capítulo 4}

\section{Elementos nilpotentes na álgebra alternativa livre com 3 geradores}

\subsection{Notação e resultados básicos}

Sejam $X_{3}=\{x, y, z\}$ e $X=\left\{x_{1}, x_{2}, \ldots\right\}$ dois conjuntos de símbolos, $\Phi$ um corpo infinito com $C h a r(\Phi) \neq 2,3$ e chamemos de $A_{3}$ e $\operatorname{Alt}[X]$ às álgebras geradas por $X_{3}$ e $X$, respectivamente, na variedade das $\Phi$-álgebras alternativas. Seja $C$ a álgebra de Cayley-Dickson sobre $\Phi$ e chamemos de $C_{7}^{(-)}$ao conjunto de elementos de $C$ com traço zero.

Notação

$$
\begin{aligned}
& (a, b, c)^{+}:=(a \circ b) \circ c-a \circ(b \circ c) \quad \text { "Associador de Jordan" } \\
& {\left[a_{1}, \ldots, a_{r+1}\right]:=\left[\left[a_{1}, \ldots, a_{r}\right], a_{r+1}\right]} \\
& \left(a_{1}, \ldots, a_{2 r-1}, a_{2 r}, a_{2 r+1}\right)^{+}:=\left(\left(a_{1}, \ldots, a_{2 r-1}\right)^{+}, a_{2 r}, a_{2 r+1}\right)^{+}
\end{aligned}
$$

Em Alt $[X]$ são satisfeitas as seguintes relações:

$$
\begin{aligned}
& 6(a, b, c)=[a, b, c]+[b, c, a]+[c, a, b] \\
& 4(a, b, c)^{+}=-2(a, b, c)+[b,[a, c]] \\
& (x, x y, z)=(x, y, z) x \\
& (x, y x, z)=x(x, y, z)
\end{aligned}
$$




$$
\begin{aligned}
& \left(x_{1} \circ x_{2}, y, z\right)=\left(x_{1}, x_{2} \circ y, z\right)+\left(x_{2}, x_{1} \circ y, z\right), \\
& (x,[y, x], z)=[x,(x, y, z)] \\
& \left(x_{1} \circ x_{2}, y, z\right)=x_{1} \circ\left(x_{2}, y, z\right)+x_{2} \circ\left(x_{1}, y, z\right) .
\end{aligned}
$$

Com efeito, (4.1) decorre do simples desenvolvimento das expressões. (4.3), (4.4), (4.5) foram provadas nos capítulos anteriores, (4.6) desprende-se diretamente de (4.3) e (4.4), a equação (4.7) é a linearização parcial de (1.4). Finalmente, (4.2) é provada observando-se que o associador é antissimétrico em seus argumentos, e que em toda álgebra se satisfaz

$4(a, b, c)^{+}=(b, a, c)+(a, b, c)+(a, c, b)-(c, b, a)-(c, a, b)-(b, c, a)+[b,[c, a]]$,

o que se verifica desenvolvendo ambos os lados da equação.

Chamaremos de $M[X]\left(M\left[X_{3}\right]\right)$ à álgebra gerada por $X\left(X_{3}\right)$ com a operação $[$,$] em Alt [X]\left(A_{3}\right)$. Notemos que pelas equações (4.1) e (4.3), $M[X]$ $\left(M\left[X_{3}\right]\right)$ é uma álgebra de Mal'tsev, isto é, nela são satisfeitas as identidades $x^{2}=0$ e $J(x, y, x z)=J(x, y, z) x$, onde $J(x, y, z)=(x y) z+(y z) x+(z x) y$ é o Jacobiano de $x, y$ e $z$.

Diremos que $f \in \operatorname{Ass}[X]$ é um $j$-polinômio se puder ser expresso a partir dos elementos de $X$, por meio de somas, produto por escalares, elevação ao quadrado e multiplicação quadrática $\left(x U_{y}=y x y\right)$. Analogamente, trocando $\operatorname{Ass}[X]$ por $\operatorname{Alt}[X]$, definimos um $j$-polinômio alternativo.

Consideremos, agora, $J_{\mathrm{Alt}}[X]$ sendo a álgebra de Jordan gerada por $X \mathrm{em}$ Alt $[X]$ (a operação é o). Então, $J_{\text {Alt }}[X] \cong S J[X]$, onde $S J[X]$ é uma álgebra especial de Jordan ([12, p.58]).

Se $\pi: \operatorname{Alt}[X] \rightarrow \operatorname{Ass}[X]$ é o homomorfismo canônico, ou seja, $\pi\left(x_{i}\right)=x_{i}$, para todo $x_{i} \in X$, então, pela própria definição e notanto que $x \circ y=$ $\frac{1}{2}\left[(x+y)^{2}-x^{2}-y^{2}\right], x^{2}=x \circ x$ e $x y x=2(x \circ y) \circ x-y \circ(x \circ x)$, temos que $S J[X]$ é o conjunto de $j$-polinômios e $J_{\text {Alt }}[X]$ é o conjunto de $j$-polinômios alternativos e portanto $\pi\left(J_{\mathrm{Alt}}[X]\right)=S J[X]$.

Se chamarmos $R_{\alpha}$ ao operador de multiplicação por $\alpha$ pela direita, em $\operatorname{Alt}[X]$, temos o seguinte resultado:

Lema 19 Se $f=f\left(x_{1}, \ldots, x_{n}\right) \in J_{\text {Alt }}[X]$, então

$$
R_{f\left(x_{1}, \ldots, x_{n}\right)}=f^{\pi}\left(R_{x_{1}}, \ldots, R_{x_{n}}\right) .
$$

Demonstração: Seja $S \subseteq J_{\text {Alt }}[X]$ o conjunto dos $j$-polinômios alternativos para os quais o lema é válido. Claramente, $X \subseteq S$. Além disso, pela lei 
alternativa direita

$$
R_{f^{2}\left(x_{1}, \ldots, x_{n}\right)}=\left(R_{f\left(x_{1}, \ldots, x_{n}\right)}\right)^{2}=\left[f^{\pi}\left(R_{x_{1}}, \ldots, R_{x_{n}}\right)\right]^{2}=\left(f^{2}\right)^{\pi}\left(R_{x_{1}}, \ldots, R_{x_{n}}\right)
$$

e pela identidade direita de Moufang

$$
\begin{aligned}
R_{f g f\left(x_{1}, \ldots, x_{n}\right)} & =R_{f\left(x_{1}, \ldots, x_{n}\right)} R_{g\left(x_{1}, \ldots, x_{n}\right)} R_{f\left(x_{1}, \ldots, x_{n}\right)} \\
& =f^{\pi}\left(R_{x_{1}}, \ldots, R_{x_{n}}\right) g^{\pi}\left(R_{x_{1}}, \ldots, R_{x_{n}}\right) f^{\pi}\left(R_{x_{1}}, \ldots, R_{x_{n}}\right) \\
& =(f g f)^{\pi}\left(R_{x_{1}}, \ldots, R_{x_{n}}\right) .
\end{aligned}
$$

De onde segue que $S$ é fechado sob elevação ao quadrado e multiplicação quadrática. É claro que $S$ também é fechado sob combinações lineares. Portanto, podemos concluir que $S=J_{\mathrm{Alt}}[X]$.

Notemos que pelo Lema 19, $\operatorname{ker} \pi \cap J_{\mathrm{Alt}}[X]=\{0\}$, pois se $f \in \operatorname{ker} \pi \cap J_{\mathrm{Alt}}[X]$, então $R_{f\left(x_{1}, \ldots, x_{n}\right)}=0$.

Pela observação anterior, vemos que as seguintes identidades, por serem $j$-polinômios, basta verificá-las em Ass $[X]$ para serem válidas em Alt $[X]$, o que é direto do desenvolvimento das expressões:

$$
\begin{aligned}
& (a, b, a)^{+}=0 \\
& (a, b, c)^{+}+(b, c, a)^{+}+(c, a, b)^{+}=0 \\
& \left(a,(x, y, z)^{+}, b\right)^{+}=\left((a, x, b)^{+}, y, z\right)^{+}+\left(x,(a, y, b)^{+}, z\right)^{+}+\left(x, y,(a, z, b)^{+}\right)^{+} \\
& (a, x \circ y, b)^{+}=(a, x, b)^{+} \circ y+(a, y, b)^{+} \circ x \\
& {\left[a, x^{2}\right]=2[a, x] \circ x}
\end{aligned}
$$

Lembremos que pelo Corolário 2 , se $u_{1}, u_{2}, v_{1}, v_{2}$ dependem somente de $x$ e $y$, então

$$
\left(u_{1}, u_{2}, z\right) \circ\left[v_{1}, v_{2}\right]=0 .
$$

Pelos teoremas 4 e 5 temos que se $a, b, c, d$, e são elementos quaisquer de $A_{3}$, então

$$
\begin{aligned}
& (a, b, c)^{2},(a, b, c) \circ[d, e] \in Z\left(A_{3}\right), \\
& {[a, b] \circ[c, d] \in N\left(A_{3}\right) .}
\end{aligned}
$$

de onde obtemos o seguinte resultado:

Lema 20 Sejam $a_{1}, \ldots, a_{r}, a, b, c, d, e \in A_{3}$, então

$$
\left[(a, b, c), a_{1}, \ldots, a_{r}\right] \circ[d, e] \in Z\left(A_{3}\right),
$$


Demonstração: Aplicaremos indução sobre $r$. Linearizando (4.12), obtemos $[a, x \circ y]=[a, x] \circ y+[a, y] \circ x$. Aqui, pela equação (4.14), temos

$$
\begin{aligned}
{\left[(a, b, c), a_{1}\right] \circ[d, e] } & =\left[a_{1},[d, e]\right] \circ(a, b, c)-\left[a_{1},[d, e] \circ(a, b, c)\right] \\
& =\left[a_{1},[d, e]\right] \circ(a, b, c) \in Z\left(A_{3}\right) .
\end{aligned}
$$

Assim, se $r=1$, o corolário é válido. Suponhamos que para $n=r-1$ se satisfaz (4.16). Usando novamente a linearização parcial de (4.12), para depois aplicar (4.15) e a hipótese de indução, temos

$$
\begin{aligned}
{\left[(a, b, c), a_{1}, \ldots, a_{r}\right] \circ[d, e]=} & {\left[\left[(a, b, c), a_{1}, \ldots, a_{r-1}\right], a_{r}\right] \circ[d, e] } \\
= & {\left[a_{r},[d, e] \circ\left[(a, b, c), a_{1}, \ldots, a_{r-1}\right]\right] } \\
& -\left[a_{r},[d, e]\right] \circ\left[(a, b, c) a_{1}, \ldots, a_{r-1}\right] \\
= & {\left[(a, b, c), a_{1}, \ldots, a_{r-1}\right] \circ[[d, e], a] \in Z\left(A_{3}\right) . }
\end{aligned}
$$

com o que acaba a demonstração.

Consideremos a álgebra $R\left(A_{3}^{(+)}\right)$das multiplicações pela direita em $A_{3}^{(+)} \mathrm{e}$ $R_{u}$ o operador multiplicação por $u$ pela direita em $A_{3}^{(+)}$, onde $A_{3}^{(+)}$é a álgebra obtida a partir de $\{x, y, z\}$ substituindo a operação usual por o.

Sejam $L_{a, b}:=R_{a} R_{b}-R_{a \circ b} \mathrm{e}$

$$
\begin{aligned}
& V_{0}=\left\{L_{a, b} \mid a, b \in X_{3}, a \neq b\right\}, \\
& V_{1}=\left\{L_{a, b} \mid a \in X_{3}\right\} .
\end{aligned}
$$

Notemos que os operadores $L_{a, b}$ são tais que $x L_{a, b}=(x, a, b)^{+}$. Denotemos por $\Lambda=\operatorname{Vect}_{\Phi}\left\{X_{3} \cup\left[X_{3}, X_{3}\right]\right\}$ e $V=\left\{L_{u, v}: u, v \in \Lambda\right\}$. Para cada $d \in X_{3}$ e $L_{a, b} \in V_{0} \cup V_{1}$, definimos

$$
L_{a, b}^{d}= \begin{cases}L_{a, b}, & \text { se } d \neq b \\ L_{b, a}, & \text { se } d=b\end{cases}
$$

\subsection{Identidades em álgebras alternativas com 3 geradores}

Nesta seção encontraremos várias novas identidades das quais precisaremos adiante para a prova do resultado principal deste capítulo. 
Lema $21 \mathrm{Em} A_{3}$ são satisfeitas as seguintes identidades:

$$
\begin{aligned}
& (x,(x, y, z), y)^{+}=0, \\
& \left(x,\left(x^{k}, y^{l}, z\right), y\right)^{+}=0, \\
& \left([y, z],\left(x^{k}, y^{l}, z\right), z\right)^{+}=0 .
\end{aligned}
$$

Demonstração: A igualdade (4.17) é conseqüência direta da equação (4.7). Por outro lado, sendo $A_{3}$ de potências associativas e aplicando (4.7) para depois aplicar (4.3) e (4.4), obtemos

$$
\begin{aligned}
\left(x^{k}, y^{l}, z\right)=\left(x^{k-1} \circ x, y^{l}, z\right) & =x \circ\left(x^{k-1}, y^{l}, z\right)+x^{k-1} \circ\left(x, y^{l}, z\right) \\
& =\left(x^{k-1}, y^{l}, z \circ x\right)+\left(x, y^{l}, z \circ x^{k-1}\right) .
\end{aligned}
$$

Desta forma, podemos diminuir $k$ e $l$ até chegar a uma soma de termos com $k=l=1$. Portanto, (4.18) segue de (4.17). Finalmente, das equações (4.7) e (4.13),

$$
\begin{aligned}
\left([y, z],\left(x^{k}, y^{l}, z\right), z\right)^{+} & =\left([y, z] \circ\left(x^{k}, y^{l}, z\right)\right) \circ z-[y, z] \circ\left(\left(x^{k}, y^{l}, z\right) \circ z\right) \\
& =\left([y, z] \circ\left(x^{k}, y^{l}, z\right)\right) \circ z-[y, z] \circ\left(x^{k}, y^{l}, z^{2}\right) \\
& =0
\end{aligned}
$$

Portanto, (4.19) e o Lema 21 estão provados.

Agora, vamos provar uma relação análoga a (4.7) mas para associadores de Jordan, restringindo-nos a certa classe de elementos.

Lema 22 Sejam $w=\left[(a, b, c), a_{1}, \ldots, a_{r}\right], a, b, c, a_{1}, \ldots, a_{r} \in A_{3}$ e $b_{1}, b_{2}, b_{3} \in$ $A_{3}$ elementos quaisquer. Então

$$
\left(w, b_{1}, b_{2}\right)^{+} \circ b_{3}=\left(b_{3}, b_{2}, b_{1}\right)^{+} \circ w+\left(w \circ b_{3}, b_{2}, b_{1}\right)^{+}
$$

e se $b_{3}=\left[b_{3}^{\prime}, b_{3}^{\prime \prime}\right]$, então

$$
\left(w, b_{1}, b_{2}\right)^{+} \circ b_{3}=\left(b_{3}, b_{2}, b_{1}\right)^{+} \circ w .
$$

Demonstração: Para começar vamos provar a seguinte igualdade:

$$
(a \circ b, c, d)^{+}=a \circ(b, c, d)^{+}+b \circ(a, c, d)^{+}+\frac{1}{4}([c, a] \circ[b, d]+[c, b] \circ[a, d])
$$


Com efeito, aplicando (4.2), (4.7) e desenvolvendo as expressões para aplicar (4.2) novamente, temos

$$
\begin{aligned}
4(a \circ b, c, d)^{+}= & -2(a \circ b, c, d)+[c,[a \circ b, d]] \\
= & -2(a \circ(b, c, d)+b \circ(a, c, d))+[c,[a \circ b, d]] \\
= & a \circ\{-2(b, c, d)+[c,[b, d]]\}+b \circ\{-2(a, c, d)+[c,[a, d]]\} \\
& +[c,[a \circ b, d]]-a \circ[c \circ[b, d]]-b \circ[c,[a, d]] \\
= & 4 a \circ(b, c, d)^{+}+4 b \circ(a, c, d)^{+}+[c,[a \circ b, d]]-a \circ[c,[b, d]] \\
& -b \circ[c,[a, d]] .
\end{aligned}
$$

Mas, pela linearização de (4.12),

$$
\begin{aligned}
{[c,[a \circ b, d]] } & =[c,[a, d] \circ b]+[c,[b, d] \circ a] \\
& =[c,[a, d]] \circ b+[c, b] \circ[a, d]+[c,[b, d]] \circ a+[c, a] \circ[b, d],
\end{aligned}
$$

de onde concluímos (4.22). Estando (4.22) provada, vamos aplicá-la junto com (4.12):

$$
\begin{aligned}
\left(w, b_{1}, b_{2}\right)^{+}= & \left(w \circ b_{3}, b_{1}, b_{2}\right)^{+}-\left(b_{3}, b_{1}, b_{2}\right)^{+} \circ w \\
& -\frac{1}{4}\left(\left[b_{1}, b_{3}\right] \circ\left[w, b_{2}\right]+\left[b_{1}, w\right] \circ\left(b_{3}, b_{2}\right)\right) \\
= & \left(w \circ b_{3}, b_{1}, b_{2}\right)^{+}-\left(b_{3}, b_{1}, b_{2}\right)^{+} \circ w \\
& -\frac{1}{4}\left\{w \circ\left[b_{2},\left[b_{1}, b_{3}\right]\right]-\left[b_{2}, w \circ\left[b_{1}, b_{3}\right]\right]\right. \\
& \left.+\left[b_{1},\left[b_{3}, b_{2}\right] \circ w\right]-\left[b_{1},\left[b_{3}, b_{2}\right]\right] \circ w\right\} .
\end{aligned}
$$

Pela equação (4.16), o quarto e o quinto termos são zero. Por outro lado, fazendo uso da equação (4.2), obtemos

$$
\begin{aligned}
4\left(b_{3}, b_{2}, b_{1}\right)^{+}+4\left(b_{3}, b_{1}, b_{2}\right)^{+}= & -2\left(b_{3}, b_{2}, b_{1}\right)-2\left(b_{3}, b_{1}, b_{2}\right) \\
& +\left[b_{2},\left[b_{3}, b_{1}\right]\right]+\left[b_{1},\left[b_{3}, b_{2}\right]\right] \\
= & {\left[b_{1}, b_{2}, b_{3}\right]+\left[b_{1},\left[b_{3}, b_{2}\right]\right] . }
\end{aligned}
$$

Substituindo isto na equação anterior, concluímos que (4.20) é satisfeita. Finalmente, observemos que (4.21) é conseqüência direta de (4.20) e (4.16).

A seguir, provaremos um resultado que será a principal ferramenta usada na próxima seção.

Proposição 4 Sejam $k, l, m \in\{1,2\}$. Então, as seguintes identidades são satisfeitas em $A_{3}$ :

$$
\left(x^{k}, y^{l}, z^{m}\right) L_{1} \cdots L_{r}=\left(x^{k}, y^{l}, z^{m} L_{1}^{z} \cdots L_{r}^{z}\right)
$$


onde $L_{i} \in V_{0}$;

$$
\left(u_{1},\left(x^{k}, y^{l}, z^{m}\right) L_{1} \cdots L_{r}, u_{2}\right)^{+}=0,
$$

onde $u_{i}=\left(a_{i_{1}}, \ldots, a_{i s_{i}}\right)^{+}, i=1,2, a_{i j} \in \Lambda, L_{i} \in V$;

$$
\left(x^{k}, y^{l}, z^{m}\right) L_{1} \cdots L_{r-1}\left[L_{r}, L_{r+1}\right]=0,
$$

onde $L_{i} \in V ; e$

$$
\left(x^{k}, y^{l}, z^{m}\right) L_{1} \cdots L_{s} \circ\left(b_{1}, \ldots, b_{r}\right)^{+}=0,
$$

onde $L_{i} \in V, b_{1}, \ldots, b_{r} \in \Lambda$ ed $\left(\left(b_{1}, \ldots, b_{r}\right)^{+}\right)>1$.

Demonstração: Procederemos por indução sobre o grau em cada uma das igualdades. Para isto, denotaremos por $(* *)_{d}$ para nos referirmos à equação $(* *)$ com grau $d$.

O grau mínimo das equações (4.23) e (4.25) é três e, neste caso, não há nada para provar. O mínimo grau de (4.24) é cinco e (4.24) 5 segue de (4.18). Por último, o grau mínimo de (4.26) é obtido quando $s=0$ e os $b_{i} \in X_{3}$. Neste caso, temos, aplicando (4.11) e (4.18), que

$$
\begin{aligned}
(x, y, z) \circ(a, x, b)^{+} & =(a, x \circ(x, y, z), b)^{+}-(a,(x, y, z), b)^{+} \circ x \\
& =\frac{1}{2}\left(a,\left(x^{2}, y, z\right), b\right)^{+}-(a,(x, y, z), b)^{+} \circ x \\
& =0 .
\end{aligned}
$$

A seguir vamos provar que as equações $(4.23)_{d},(4.24)_{d}$ e $(4.25)_{d}$ seguem de $(4.26)_{d}$. Se $L_{r}=L_{a_{r}, b_{r}}$, então, usando (4.9) e (4.24) ${ }_{d-1}$ (hipótese de indução) junto com a linearização parcial de (4.8), temos

$$
\begin{aligned}
\left(x, y^{l},\right. & \left.z^{m}\right) L_{1} \cdots L_{r} \circ x \\
& \left.=\left(x, y^{l}, z^{m}\right) L_{1} \cdots L_{r-1}, a_{r}, b_{r}\right)^{+} \circ x \\
& =\left\{-\left(a_{r}, b_{r},\left(x, y^{l}, z^{m}\right) L_{1} \cdots L_{r-1}\right)^{+}-\left(b_{r},\left(x, y^{l}, z^{m}\right) L_{1} \cdots L_{r-1}, a_{r}\right)^{+}\right\} \circ x \\
& =\left(\left(x, y^{l}, z^{m}\right) L_{1} \cdots L_{r-1}, b_{r}, a_{r}\right)^{+} \circ x .
\end{aligned}
$$

Usemos agora (4.20) e (4.26) ${ }_{d}$,

$$
\begin{aligned}
\left(\left(x, y^{l}, z^{m}\right) L_{1} \cdots L_{r-1}, b_{r}, a_{r}\right)^{+} \circ x= & \left(x, a_{r}, b_{r}\right)^{+} \circ\left(x, y^{l}, z^{m}\right) L_{1} \cdots L_{r-1} \\
& +\left(\left(x, y^{l}, z^{m}\right) L_{1} \cdots L_{r-1} \circ x, a_{r}, b_{r}\right)^{+} \\
= & \left(\left(x, y^{l}, z^{m}\right) L_{1} \cdots L_{r-1} \circ x, a_{r}, b_{r}\right)^{+} \\
= & \left(\left(x, y^{l}, z^{m}\right) L_{1} \cdots L_{r-1} \circ x\right) L_{r} .
\end{aligned}
$$


Continuando este processo para depois aplicar (4.7), concluímos que

$$
\left(x, y^{l}, z^{m}\right) L_{1} \cdots L_{r-1} \circ x=\frac{1}{2}\left(x^{2}, y^{l}, z^{m}\right) L_{1} \cdots L_{r} .
$$

Agora, se em $(4.24)_{d}, k, l$ ou $m$ é maior que 1 , podemos, pela simetria, supor que $k=2$ e, neste caso, aplicando (4.27) $d$ e (4.11), obtemos

$$
\begin{aligned}
\left(u_{1},\left(x^{2}, y^{l}, z^{m}\right) L_{1} \cdots L_{r}, u_{2}\right)^{+}= & 2\left(u_{1}, x \circ\left(x, y^{l}, z^{m}\right) L_{1} \cdots L_{r}, u_{2}\right)^{+} \\
= & 2 x \circ\left(u_{1},\left(x, y^{l}, z^{m}\right) L_{1} \cdots L_{r}, u_{2}\right)^{+} \\
& +2\left(x, y^{l}, z^{m}\right) \circ\left(u_{1}, x, u_{2}\right)^{+} .
\end{aligned}
$$

Daí, decorre, por $(4.24)_{d-1}$ e $(4.26)_{d}$, que $\left(u_{1},\left(x^{2}, y^{l}, z^{m}\right) L_{1} \cdots L_{r}, u_{2}\right)^{+}=0$. Assim, para $k, l$ ou $m$ diferentes de $1,(4.24)_{d}$ segue da equação $(4.26)_{d}$. Analisemos agora o caso em que $k=l=m=1$. Se $d\left(u_{1}\right)>1$ e $d\left(u_{2}\right)=1$, podemos supor $u_{2}=x$ e, neste caso, fazendo uso de $(4.26)_{d-1},(4.27)_{d-1} \mathrm{e}$ $(4.26)_{d}$, temos:

$$
\begin{aligned}
\left(u_{1},(x, y, z) L_{1} \cdots L_{r}, x\right)^{+} & =\left(u_{1} \circ(x, y, z) L_{1} \cdots L_{r}\right) \circ x-u_{1} \circ\left((x, y, z) L_{1} \cdots L_{r} \circ x\right) \\
& =-u_{1} \circ\left((x, y, z) L_{1} \cdots L_{r} \circ x\right) \\
& =-\frac{1}{2} u_{1} \circ\left(x^{2}, y, z\right) L_{1} \cdots L_{r}=0 .
\end{aligned}
$$

Se $d\left(u_{1}\right)=1$ e $d\left(u_{2}\right)>1$, a demonstração é análoga. Suponhamos agora que $d\left(u_{1}\right)=d\left(u_{2}\right)=1$. Sem restrição, podemos supor $u_{1}=x$ e $u_{2}=y$. Neste caso, pela equação $(4.27)_{d}$,

$$
\begin{aligned}
\left(x,(x, y, z) L_{1} \cdots L_{r}, y\right)^{+} & =\left(x \circ(x, y, z) L_{1} \cdots L_{r}\right) \circ y-x \circ\left((x, y, z) L_{1} \cdots L_{r} \circ y\right) \\
& =\frac{1}{4}\left\{\left(x^{2}, y^{2}, z\right) L_{1} \cdots L_{r}-\left(x^{2}, y^{2}, z\right) L_{1} \cdots L_{r}\right\} \\
& =0 .
\end{aligned}
$$

Finalmente, se $d\left(u_{1}\right)>1$ e $d\left(u_{2}\right)>1$, então segue de (4.26) $)_{d-1}$ que

$$
\begin{aligned}
\left(u_{1},(x, y, z) L_{1} \cdots L_{r}, u_{2}\right)^{+}= & \left(u_{1} \circ(x, y, z) L_{1} \cdots L_{r}\right) \circ u_{2} \\
& -u_{1} \circ\left((x, y, z) L_{1} \cdots L_{r} \circ u_{2}\right) \\
= & 0
\end{aligned}
$$

Assim, provamos que $(4.24)_{d}$ depende de $(4.26)_{d}$.

Consideremos agora $(4.25)_{d}$. Dado $w=\left(x^{k}, y^{l}, z^{m}\right) L_{1} \cdots L_{r-1}$, usando a linearização parcial de (4.8) (antissimetria do associador de Jordan com 
respeito ao primeiro e ao terceiro argumentos) e as equações (4.9) e $(4.24)_{d}$, temos que $(w, a, b)^{+}=-(a, b, w)^{+}-(b, w, a)^{+}=(w, b, a)^{+}$. Portanto, $w L_{a, b}=$ $w L_{b, a}, a, b \in \Lambda$. Por esta razão, se tivermos $w\left[L_{a, a}, L_{b, b}\right]=0$, para quaisquer $a, b \in \Lambda$, então, como $L_{a, b}=\frac{1}{2}\left(L_{a, b}+L_{b, a}\right)$,

$$
\begin{aligned}
w\left[L_{a, b}, L_{c, d}\right] & =w L_{a, b} L_{c, d}-w L_{c, d} L_{a, b} \\
& =\frac{1}{4} w\left\{\left(L_{a, b}+L_{b, a}\right)\left(L_{c, d}+L_{d, c}\right)+\left(L_{c, d}+L_{d, c}\right)\left(L_{a, b}+L_{b, a}\right)\right\} .
\end{aligned}
$$

Mas $L_{x, y}+L_{y, x}=L_{x+y, x+y}-L_{x, x}-L_{y, y}$. Daí, $w\left[L_{a, b}, L_{c, d}\right]=0$. Portanto, para provar (4.25) $)_{d}$ é suficiente prová-lo para $L_{r}=L_{a, a}$ e $L_{r+1}=L_{b, b}, a, b \in \Lambda$.

Agora, usando (4.10) e depois $(4.24)_{d}$,

$$
\begin{aligned}
w L_{a, a} L_{b, b} & =\left((w, a, a)^{+}, b, b\right)^{+} \\
& =-\left(a,(w, b, a)^{+}, b\right)^{+}-\left(a, b,(w, b, a)^{+}\right)^{+}+\left(w,(a, b, b)^{+}, a\right)^{+} \\
& =\left((w, b, a)^{+}, b, a\right)^{+}+\left(w,(a, b, b)^{+}, a\right)^{+}
\end{aligned}
$$

Mas, como já mostramos, $\widetilde{w} L_{a, b}=\widetilde{w} L_{b, a}$, para os $\widetilde{w} \in A_{3}$ que têm a forma $\widetilde{w}=\left(x^{k}, y^{l}, z^{m}\right) L_{1} \cdots L_{s}$. Daqui, usando (4.10), (4.24) $)_{d}$ e (4.10) novamente, temos

$$
\begin{aligned}
w L_{a, a} L_{b, b}= & \left((w, a, b)^{+}, a, b\right)^{+}+\left(w,(a, b, b)^{+}, a\right)^{+} \\
= & -\left(a,(w, a, b)^{+}, b\right)^{+}-\left(a, a,(w, b, b)^{+}\right)^{+}+\left(w,(a, a, b)^{+}, b\right)^{+} \\
& +\left(w,(a, b, b)^{+}, a\right)^{+} \\
= & \left((w, b, b)^{+}, a, a\right)^{+}+\left(w,(a, a, b)^{+}, b\right)^{+}+\left(w,(a, b, b)^{+}, a\right)^{+} \\
= & \left((w, b, b)^{+}, a, a\right)^{+}+\left((a, w, b)^{+}, a, b\right)^{+}-\left(w, a,(a, b, b)^{+}\right)^{+} \\
& +\left(a,(w, a, b)^{+}, b\right)^{+}+\left(w,(a, b, b)^{+}, a\right)^{+} .
\end{aligned}
$$

Por causa de $(4.24)_{d}$, o segundo e o quarto termos se anulam. Além disso, por $(4.9)$ e $(4.24)_{d}$

$$
\begin{aligned}
-\left(w, a,(a, b, b)^{+}\right)^{+}+\left(w,(a, b, b)^{+}, a\right)^{+} & =\left((a, b, b)^{+}, a, w\right)^{+}+\left(w,(a, b, b)^{+}, a\right)^{+} \\
& =-\left(a, w,(a, b, b)^{+}\right)^{+} \\
& =0
\end{aligned}
$$

de onde segue $\left((w, a, a)^{+}, b, b\right)^{+}=\left((w, b, b)^{+}, a, a\right)^{+}$, ou seja, $w\left[L_{a, a}, L_{b, b}\right]=0$, como queríamos provar. 
Consideremos agora $(4.23)_{d}$. Podemos supor $L_{r}=L_{x, z}$. Aplicando (4.9), (4.8), $(4.24)_{d}$ e $(4.23)_{d-1}$, nessa ordem, e finalmente pela observação anterior,

$$
\begin{aligned}
\left(x^{k}, y^{l}, z^{m}\right) L_{1} \cdots L_{r-1} L_{x, z}= & \left(\left(x^{k}, y^{l}, z^{m}\right) L_{1} \cdots L_{r-1}, x, z\right)^{+} \\
= & -\left(x, z,\left(x^{k}, y^{l}, z^{m}\right) L_{1} \cdots L_{r-1}\right)^{+} \\
& -\left(z,\left(x^{k}, y^{l}, z^{m}\right) L_{1} \cdots L_{r-1}, x\right)^{+} \\
= & \left(\left(x^{k}, y^{l}, z^{m}\right) L_{1} \cdots L_{r-1}, z, x\right)^{+} \\
& -\left(z,\left(x^{k}, y^{l}, z^{m}\right) L_{1} \cdots L_{r-1}, x\right)^{+} \\
= & \left(\left(x^{k}, y^{l}, z^{m}\right) L_{1} \cdots L_{r-1}, z, x\right)^{+} \\
= & \left(x^{k}, y^{l}, z^{m} L_{1} \cdots L_{r-1}\right) L_{z, x} \\
= & \left(x^{k}, y^{l}, z^{m} L_{1} \cdots L_{r-1}\right) L_{x, z} .
\end{aligned}
$$

Para simplificar, chamemos $z^{m} L_{1} \cdots L_{r-1}=u$. Da equação (4.7), o teorema de Artin e (4.9), segue

$$
\begin{aligned}
\left(x^{k}, y^{l},(u, x, z)^{+}\right) & =\left(x^{k}, y^{l},(u \circ x) \circ z\right)-\left(x^{k}, y^{l}, u \circ(x \circ z)\right) \\
& =\left(\left(x^{k}, y^{l}, u\right), x, z\right)^{+}-\left(\left(x^{k}, y^{l}, z\right), x, u\right)^{+} \\
& =\left(\left(x^{k}, y^{l}, u\right), x, z\right)^{+}+\left(x, u,\left(x^{k}, y^{l}, z\right)\right)^{+}+\left(u,\left(x^{k}, y^{l}, z\right), x\right)^{+} .
\end{aligned}
$$

Daqui e usando $(4.23)_{d-1},(4.11),(4.9)$ e $(4.24)_{d}$, obtemos

$$
\begin{aligned}
\left(x^{k}, y^{l}, z^{m}\right) L_{1} \cdots L_{r-1} L_{x, z}= & \left(x^{k}, y^{l}, z^{m} L_{1} \cdots L_{r-1}\right) L_{x, z} \\
= & -\left(x^{k}, y^{l},\left(z^{m} L_{1} \cdots L_{r-1}, x, z\right)^{+}\right) \\
& -\left(x, z^{m} L_{1} \cdots L_{r-1},\left(x^{k}, y^{l}, z\right)\right)^{+} \\
& -\left(z^{m} L_{1} \cdots L_{r-1},\left(x^{k}, y^{l}, z\right), x\right)^{+} \\
= & \left(x^{k}, y^{l}, z^{m} L_{1} \cdots L_{r}\right) .
\end{aligned}
$$

Portanto, $(4.23)_{d}$ decorre de $(4.26)_{d}$. Provamos assim que para provar a proposição é suficiente provar a equação $(4.26)_{d}$, o que faremos por indução sobre $d$.

Pela equação (4.20), podemos supor que $s=0$. Também podemos supor que os $b_{j} \in X_{3} \cup\left[X_{3}, X_{3}\right]$. Se ao menos dois dos $b_{j}$ 's pertencem a $\left[X_{3}, X_{3}\right]$, então podemos supor $b_{1} \in\left[X_{3}, X_{3}\right]$, já que é claro, das equações (4.9) e (4.10), que o conjunto $\left\{\left(x_{1}, x_{i_{2}}, \ldots, x_{i_{2 n+1}}\right) \mid\left\{i_{2}, \ldots, i_{2 n+1}\right\}=\{2, \ldots, 2 n+1\}\right\}$ gera o espaço vetorial formado pelos associadores de Jordan em $x_{1}, x_{2}, \ldots, x_{2 n+1}$. Além disso, se escrevemos $\left(b_{1}, \ldots, b_{r}\right)^{+}=b_{1} L_{1} \ldots, L_{p}$, aplicando $(4.25)_{d-1} \mathrm{e}$ 
$(4.24)_{d-1}$ podemos supor que $b_{3} \in\left[X_{3}, X_{3}\right]$. Usando agora $(4.24)_{d-1},(4.21)$, (4.11) e (4.16) juntos para finalizar com $(4.24)_{d-1}$, temos

$$
\begin{aligned}
\left(x^{k}, y^{l}, z^{m}\right) \circ b_{1} L_{b_{2}, b_{3}} L_{2} \ldots L_{p} & =\left(x^{k}, y^{l}, z^{m}\right) \circ b_{1} L_{b_{3}, b_{2}} L_{2} \cdots L_{p} \\
& =\left(x^{k}, y^{l}, z^{m}\right) L_{p} \cdots L_{2} \circ b_{1} L_{b_{3}, b_{2}} \\
& =-b_{3} \circ\left(b_{1},\left(x^{k}, y^{l}, z^{m}\right) L_{p} \cdots L_{2}, b_{2}\right)^{+} \\
& =0 .
\end{aligned}
$$

É claro que se existe um único $j>3$ tal que $b_{j} \in\left[X_{3}, X_{3}\right]$, a prova continua válida. Por último, pelas equações (4.9) e (4.8), basta considerar o caso em que $b_{2}, \ldots, b_{r} \in X_{3}$ e $b_{1} \in X_{3} \cup\left[X_{3}, X_{3}\right]$. Suponhamos que $k, l$ ou $m$ é diferente de 1. Pela simetria, podemos supor $k=2 \mathrm{e}$, neste caso, aplicando (4.7) e (4.26) $)_{d-1}$,

$$
\begin{aligned}
& \left(x^{2}, y^{l}, z^{m}\right) \circ\left(b_{1}, \ldots, b_{r}\right)^{+}=2\left(x \circ\left(x, y^{l}, z^{m}\right)\right) \circ\left(b_{1}, \ldots, b_{r}\right)^{+} \\
& =2\left(x \circ\left(x, y^{l}, z^{m}\right)\right) \circ\left(b_{1}, \ldots, b_{r}\right)^{+}+2 x \circ\left(\left(x, y^{l}, z^{m}\right) \circ\left(b_{1}, \ldots, b_{r}\right)^{+}\right) \\
& =2\left(x,\left(x, y^{l}, z^{m}\right),\left(b_{1}, \ldots, b_{r}\right)\right)^{+}
\end{aligned}
$$

Deste modo, $(4.26)_{d}$, com $k, l$ ou $m$ igual a 2 , se reduz a $(4.24)_{d}$, com $u_{1} \in X_{3}$. Caso contrário, se $k=l=m=1$, podemos, sem restrição, supor que $b_{r-1}=x$ e aplicando as equações $(4.11),(4.7)$ e $(4.24)_{d-1}$, obtemos

$$
\begin{aligned}
(x, y, z) & \circ\left(b_{1}, \ldots, x, b_{r}\right)^{+} \\
& =-x \circ\left(\left(b_{1}, \ldots, b_{r-2}\right)^{+},(x, y, z), b_{r}\right)^{+}+\left(\left(b_{1}, \ldots, b_{r-2}\right)^{+}, x \circ(x, y, z), b_{r}\right)^{+} \\
& =-x \circ\left(\left(b_{1}, \ldots, b_{r-2}\right)^{+},(x, y, z), b_{r}\right)^{+}+\frac{1}{2}\left(\left(b_{1}, \ldots, b_{r-2}\right)^{+},\left(x^{2}, y, z\right), b_{r}\right)^{+} \\
& =\frac{1}{2}\left(\left(b_{1}, \ldots, b_{r-2}\right)^{+},\left(x^{2}, y, z\right), b_{r}\right)^{+} .
\end{aligned}
$$

Portanto, o caso $k=l=m=1$ se reduz a $(4.24)_{d}$ com $u_{2} \in X_{3}$.

Para provar $(4.24)_{d}$, no caso particular em que $u_{1}$ (ou $u_{2}$ ) $\in X_{3}$, vamos precisar da seguinte identidade, a qual demonstraremos usando $(4.26)_{d-1}$ :

$$
\begin{aligned}
\left(u_{1},\left(x^{k}, y^{l}, z^{m}\right) L_{1} \cdots L_{s} L_{a, b}, u_{2}\right)^{+}= & \left(u_{1} L_{a, b},\left(x^{k}, y^{l}, z^{m}\right) L_{1} \cdots L_{s}, u_{2}\right)^{+} \\
+ & \left(u_{1},\left(x^{k}, y^{l}, z^{m}\right) L_{1} \cdots L_{s}, u_{2} L_{a, b}\right)^{+}(4.28)
\end{aligned}
$$

onde $u_{i}, L_{i}, k, l, m$ são como em (4.24) e $a, b \in \Lambda$.

Se $d\left(u_{1}\right)>1$ e $d\left(u_{2}\right)>1$, então ambos os lados da equação (4.28) são zero pela equação $(4.24)_{d-1}$. Consideremos o caso em que $d\left(u_{1}\right)=d\left(u_{2}\right)=1$, 
podemos supor $u_{1}=x, u_{2}=y$. Aplicando (4.27) $)_{d-1}$ e o teorema de Artin, obtemos

$$
\begin{aligned}
\left(x \circ\left(x^{k}, y^{l}, z^{m}\right) L_{1} \cdots L_{s} L_{a, b}\right) \circ y= & \left(x^{k}, y^{l}, z^{m} \circ x\right) L_{1} \cdots L_{s} L_{a, b} \circ y \\
& -\left(z^{m} \circ\left(x^{k}, y^{l}, x\right) L_{1} \cdots L_{s}, L_{a, b}\right) \circ y \\
= & \left(x^{k}, y^{l}, z^{m} \circ x\right) L_{1} \cdots L_{s} L_{a, b} \circ y .
\end{aligned}
$$

Agora, usando a equação (4.20), e depois (4.7) e (4.27) $)_{d-1}$ juntas,

$$
\begin{aligned}
\left(x^{k}, y^{l}, z^{m}\right. & \circ x) L_{1} \cdots L_{s} L_{a, b} \circ y \\
& =\left(\left(x^{k}, y^{l}, z^{m} \circ x\right) L_{1} \cdots L_{s} \circ y\right) L_{a, b}+\left(x^{k}, y^{l}, z^{m} \circ x\right) L_{1} \cdots L_{s} \circ y L_{a, b} \\
& =\left(\left(\left(x^{k}, y^{l}, z^{m}\right) L_{1} \cdots L_{s} \circ x\right) \circ y\right) L_{a, b}+\left(x,\left(x^{k}, y^{l}, z^{m}\right) L_{1} \cdots L_{s}, y L_{a, b}\right)^{+}
\end{aligned}
$$

Do mesmo modo,

$$
\begin{aligned}
\left.x \circ\left(\left(x^{k}, y^{l}, z^{m}\right) L_{1} \cdots L_{s} L_{a, b}\right) \circ y\right)= & \left.\left(\left(x^{k}, y^{l}, z^{m}\right) L_{1} \cdots L_{s} \circ y\right) \circ x\right) L_{a, b} \\
& +\left(y,\left(x^{k}, y^{l}, z^{m}\right) L_{1} \cdots L_{s}, x L_{a, b}\right)^{+} .
\end{aligned}
$$

Subtraindo ambas as equações e aplicando $(4.24)_{d}-1$, segue (4.28). Notemos que o mesmo esquema é válido para o caso em que $d\left(u_{1}\right)=1$ e $d\left(u_{2}\right)>1 \mathrm{com}$ o qual fica provada a identidade (4.28). Vimos que para provar a proposição é suficiente provar (4.24) no caso particular em que $u_{2} \in X_{3}$. Pela simetria do problema, podemos supor $u_{2}=x$ e pela igualdade (4.18) não é necessário analisar o caso $d\left(u_{1}\right)=1$. Além disso pela equação (23) $)_{d}$, podemos supor $u_{1}=(a, b, c)^{+}$ou $u_{1}=[a, b], a, b, c \in X_{3}$.

Analisemos primeiro o caso em que exista um operador de $V_{1}$ entre $L_{1}, . ., L_{r}$. Pela equação (4.25) ${ }_{d-1}$, podemos supor $L_{r} \in V_{1}$. Se $L_{r}=L_{y, y}$ (ou $L_{r}=L_{z, z}$ ), chamemos $w=\left(x^{k}, y^{l}, z^{m}\right) L_{1} \cdots L_{r-1}$, aplicando $(4.28)_{d},(4.26)_{d-1}$ e (4.10), temos

$$
\begin{aligned}
\left(u, w L_{y, y}, x\right)^{+} & =\left(u L_{y, y}, w, x\right)^{+}+\left(u, w, x L_{y, y}\right)^{+} \\
& =\left(u L_{y, y}, w, x\right)^{+}=\left((u, y, y)^{+}, w, x\right)^{+} \\
& =-\left(y,(u, w, y)^{+}, x\right)^{+}-\left(y, w,(u, x, y)^{+}\right)^{+}+\left(u,(y, w, x)^{+}, y\right)^{+} .
\end{aligned}
$$

Usando agora as equações $(4.24)_{d-1},(4.8),(4.28)_{d}$ e novamente (4.8),

$$
\begin{aligned}
\left(u, w L_{y, y}, x\right)^{+} & =-\left(y, w,(u, x, y)^{+}\right)^{+} \\
& =\left((u, x, y)^{+}, w, y\right)^{+} \\
& =\left(u, w L_{x, y}, y\right)^{+}-\left(u, w, L_{x, y}\right)^{+} \\
& =\left(u, w L_{x, y}, y\right)^{+}
\end{aligned}
$$


eliminando assim o operador $L_{y, y}$, transformando-o em $L_{x y}$ mediante a mudança de $u_{2}=x$ por $u_{2}=y$. Assim, vemos que podemos eliminar os termos da forma $L_{y, y}$ e $L_{z, z}$ de $L_{1}, \ldots, L_{r}$. Portanto, pela equação (4.25) ${ }_{d-1}$ podemos supor que $L_{1}, \ldots, L_{j} \in V_{0}$ e $L_{j+1}=\cdots=L_{r}=L_{x, x}$.

Agora analisemos o caso em que $u$ depende somente de $x$ e $y$. Neste caso, aplicando $(4.23)_{d-1}$, temos

$$
\left(u,\left(x^{k}, y^{l}, z^{m}\right) L_{1} \cdots L_{r}, x\right)^{+}=\left(u,\left(x^{k}, y^{l}, z^{m} L_{1} \cdots L_{j} L_{x, x}^{r-j}\right), x\right)^{+} .
$$

Temos apenas dois casos para verificar, $u=[a, b]$ ou $u=(a, b, c)^{+}$. No segundo caso, como $\{a, b, c\} \subseteq\{x, y\}$, aplicando a equação (4.2), temos $u=$ $\frac{1}{4}[b,[a, c]]$. Em ambos os casos, pela equação (4.13),

$$
u \circ\left(x^{k}, y^{l}, z^{m} L_{1} \cdots L_{j} L_{x, x}^{r-j}\right)=0 .
$$

Além disso, pela equação (4.7) e o teorema de Artin,

$$
\begin{aligned}
\left(x^{k}, y^{l}, z^{m} L_{1} \cdots L_{j} L_{x, x}^{r-j}\right) \circ x= & \left(x^{k}, y^{l}, z^{m} L_{1} \cdots L_{j} L_{x, x}^{r-j} \circ x\right) \\
& -\left(x^{k}, y^{l}, x\right) \circ z^{m} L_{1} \cdots L_{j} L_{x, x}^{r-j} \\
= & \left(x^{k}, y^{l}, z^{m} L_{1} \cdots L_{j} L_{x, x}^{r-j} \circ x\right) .
\end{aligned}
$$

Daí, segue como conseqüência de (4.13), que $\left(u,\left(x^{k}, y^{l}, z^{m}\right) L_{1} \cdots L_{r}, x\right)^{+}=0$.

Suponhamos agora que $u$ também depende de $z$. É suficiente analisar os seguintes casos; $u \in\left\{(x, x, z)^{+},[x, z],[y, z],(x, y, z)^{+},(y, y, z)^{+}\right\}$.

\section{$1^{\circ}$ caso}

Se $u=(x, x, z)^{+}$, então aplicando a equação (4.28), temos que

$$
\left((x, x, z)^{+},\left(x^{k}, y^{l}, z^{m} L_{1} \cdots L_{r}\right), x\right)^{+}=\left(z,\left(x^{k}, y^{l}, z^{m} L_{1} \cdots L_{r} L_{x, x}\right), x\right)^{+},
$$

o que já foi analisado.

\section{$2^{\circ}$ caso}

Se $u=[x, z]$, usamos $(4.23)_{d-1}$ no segundo argumento, obtendo

$$
\left([x, z],\left(x^{k}, y^{l}, z^{m}\right) L_{1} \cdots L_{r}, x\right)^{+}=-\left([x, z],\left(x^{k}, z^{m}, y^{l} L_{1} \cdots L_{r}\right), x\right)^{+}
$$

ficando assim numa forma análoga a uma das já estudadas. 


\section{$3^{\circ}$ caso}

Se $u=[y, z]$, podemos supor que $L_{1} \in\left\{L_{z, x}, L_{x, x}, L_{y, z}\right\}$, já que pela simetria do problema com respeito a $y \leftrightarrow z$, não é preciso considerar $L_{1}=$ $L_{x, y}$ e os operadores $L_{y, y}$ e $L_{z, z}$ pertencem ao espaço vetorial gerado por $\left\{L_{y, z}, L_{z, y}\right\}$. Para $l=1$, aplicando $(4.23)_{d-1}$,

$$
\left([y, z],\left(x^{k}, y, z^{m}\right) L_{1} \cdots L_{r}, x\right)^{+}=\left([x, y],\left(x^{k}, y L_{i}^{y} \cdots L_{r}^{y}, z^{m}\right), x\right)^{+} .
$$

Agora, se linearizamos (4.19) com respeito à variável $z$, obtemos

$$
\left([y, w],\left(x^{k}, y^{l}, z\right), x\right)^{+}+\left([y, z],\left(x^{k}, y^{l}, w\right), x\right)^{+}=0 .
$$

Substituindo nesta equação $y=z, w=y L_{1}^{y} \cdots L_{r}^{y}, l=m$ e $z=y$, segue

$$
\left([y, z],\left(x^{k}, y, z^{m}\right) L_{1} \cdots L_{r}, x\right)^{+}=-\left(\left[y L_{1}^{y} \cdots L_{r}^{y}, z\right],\left(x^{k}, y, z^{m}\right), x\right)^{+}
$$

A seguir, provaremos por indução que

$$
\begin{aligned}
{\left[y L_{1}^{y} \cdots L_{r}^{y}, z\right]=} & {\left[y L_{1}^{y}, z\right] L_{2}^{y} \cdots L_{r}^{y}+\sum_{i=2}^{r} y L_{1}^{y} \cdots L_{c_{i},\left[d_{i}, z\right]} \cdots L_{r}^{y} } \\
& +\sum_{i=2}^{r} y L_{1}^{y} \cdots L_{\left[c_{i}, z\right], d_{i}} \cdots L_{r}^{y} .
\end{aligned}
$$

Se $r=1$, não há o que provar. Além disso, se $L_{r}=L_{c_{r}, d_{r}}$, então, pela linearização parcial de (4.12),

$$
\begin{aligned}
{\left[u L_{c_{r}, d_{r}}, z\right] } & =\left[\left(u \circ c_{r}\right) \circ d_{r}, z\right]-\left[u \circ\left(c_{r} \circ d_{r}\right), z\right] \\
& =\left([u, z], c_{r}, d_{r}\right)^{+}+u L_{c_{r},\left[d_{r}, z\right]}+u L_{\left[c_{r}, z\right], d r} .
\end{aligned}
$$

Substituindo $u=y L_{1} \cdots L_{r-1}$ e aplicando a hipótese de indução, a afirmação fica provada. Verifiquemos agora que para cada $i$,

$$
\left(y L_{1}^{y} \cdots L_{c_{i},\left[d_{i}, z\right]} \cdots L_{r}^{y},\left(x^{k}, y, z^{m}\right), x\right)^{+}=0 .
$$

Se $k=1$, então por $(4.26)_{d-1}$, no caso em que algum $b_{j} \in\left[X_{3}, X_{3}\right]$, com $j>1$,

$$
\begin{aligned}
\left(y L_{1}^{y} \cdots L_{c_{i},\left[d_{i}, z\right]} \cdots L_{r}^{y},\left(x, y, z^{m}\right), x\right)^{+}= & \left(y L_{1}^{y} \cdots L_{c_{i},\left[d_{i}, z\right]} \cdots L_{r}^{y} \circ\left(x, y, z^{m}\right)\right) \circ x \\
& -\frac{1}{2} y L_{1}^{y} \cdots L_{c_{i},\left[d_{i}, z\right]} \cdots L_{r}^{y} \circ\left(x^{2}, y, z^{m}\right) \\
= & 0 .
\end{aligned}
$$


Do mesmo modo, se $k=2$, escrevendo $t=y L_{1}^{y} \cdots L_{c_{i}\left[d_{i}, z\right]} \cdots L_{r}^{y}$ e aplicando (4.7), (4.11), (4.24) $)_{d-1}$ e (4.26) $)_{d-1}$, obtemos

$$
\begin{aligned}
\left(t,\left(x^{2}, y, z^{m}\right), x\right)^{+} & =2\left(t, x \circ\left(x, y, z^{m}\right), x\right)^{+} \\
& =2\left\{x \circ\left(t,\left(x, y, z^{m}\right), x\right)^{+}+\left(x, y, z^{m}\right) \circ(t, x, x)^{+}\right\} \\
& =0 .
\end{aligned}
$$

Com isto, provamos que

$$
\left([y, z],\left(x^{k}, y, z^{m}\right) L_{1} \cdots L_{r}, x\right)^{+}=\left(\left[y L_{1}^{y}, z\right] L_{2}^{y} \cdots L_{r}^{y},\left(x^{k}, y, z^{m}\right), x\right)^{+} .
$$

Novamente, pela linearização parcial de (4.12), obtemos

$$
\left[y L_{a, b}, z\right]=(y, a,[b, z])^{+}+(y,[a, z], b)^{+}+([y, z], a, b)^{+} .
$$

Daí,

$$
\left[y L_{1}^{y}, z\right]= \begin{cases}([y, z], z, x)^{+}+(y, z,[x, z])^{+}, & \text {se } L_{1}^{y}=L_{z, x} \\ ([y, z], x, x)^{+}+(y,[x, z], x)^{+}+(y, x,[x, z])^{+}, & \text {se } L_{1}^{y}=L_{x, x} \\ ([x, z], y, z)^{+}+(y,[y, z], x)^{+}, & \text {se } L_{1}^{y}=L_{y, z}\end{cases}
$$

Consideremos o caso $L_{1}^{y}=L_{x, x}$. Os demais são análogos e até mais simples. Da equação (4.9), segue que $(y,[x, z], x)^{+}=[x, z]\left(L_{y, x}-L_{x, y}\right)$. Daí e usando as equações $(4.28)_{d}$ e $(4.26)_{d-1}$, obtemos

$$
\begin{aligned}
\left((y,[x, z], x)^{+} L_{2}^{y} \cdots L_{r}^{y},\left(x^{k}, y, z^{m}\right), x\right)^{+} & \\
= & \left([x, z]\left(L_{y x}-L_{x y}\right) L_{2}^{y} \cdots L_{r-1}^{y},\left(x^{k}, y, z^{m}\right) L_{r}, x\right)^{+} \\
& \quad-\left([x, z]\left(L_{y x}-L_{x y}\right) L_{2}^{y} \cdots L_{r-1}^{y},\left(x^{k}, y, z^{m}\right), x L_{r}\right)^{+} \\
= & \left([x, z]\left(L_{y x}-L_{x y}\right) L_{2}^{y} \cdots L_{r-1}^{y},\left(x^{k}, y, z^{m}\right) L_{r}, x\right)^{+}
\end{aligned}
$$

Continuando este processo e pela equação $(4.24)_{d-1}$,

$$
\begin{aligned}
\left((y,[x, z], x)^{+} L_{2}^{y} \cdots L_{r}^{y},\left(x^{k}, y, z^{m}\right), x\right)^{+} & =\left([x, z],\left(y,\left(x^{k}, y, z^{m}\right) L_{2}^{y} \cdots L_{r}^{y}, x\right)^{+}, x\right)^{+} \\
& =0 .
\end{aligned}
$$

Por outro lado, aplicando (4.8), (4.28) $)_{d}$ e (4.26) $)_{d-1}$,

$$
\begin{aligned}
\left((y, x,[x, z])^{+} L_{2}^{y} \cdots L_{r}^{y},\left(x^{k}, y, z^{m}\right), x\right)^{+} & \\
& =-\left(([x, z], x, y)^{+} L_{2}^{y} \cdots L_{r}^{y},\left(x^{k}, y, z^{m}\right), x\right)^{+}
\end{aligned}
$$




$$
\begin{aligned}
= & -\left(\left([x, z] L_{x, y} L_{2}^{y} \cdots L_{r-1}^{y},\left(x^{k}, y, z^{m}\right) L_{r}^{y}, x\right)^{+}\right. \\
& +\left([x, z] L_{x, y} L_{2}^{y} \cdots L_{r-1}^{y},\left(x^{k}, y, z^{m}\right), x L_{r}^{y}\right)^{+} \\
= & -\left([x, z] L_{x, y} L_{2}^{y} \cdots L_{r-1}^{y},\left(x^{k}, y, z^{m}\right) L_{r}^{y}, x\right)^{+}
\end{aligned}
$$

Continuando com o mesmo esquema e depois aplicando $(4.25)_{d-1},(4.23)_{d-1}$ e $(4.28)_{d}$, obtemos

$$
\begin{aligned}
\left((y, x,[x, z])^{+} L_{2}^{y} \cdots L_{r}^{y},\left(x^{k}, y, z^{m}\right), x\right)^{+} & =-\left(\left([x, z],\left(x^{k}, y, z^{m}\right) L_{r}^{y} \cdots L_{2}^{y} L_{x y}^{y}, x\right)^{+}\right. \\
& =-\left(\left([x, z],\left(x^{k}, y, z^{m}\right) L_{2}^{y} \cdots L_{j}^{y} L_{x y} L_{x x}^{r-j}, x\right)^{+}\right. \\
& =-\left(\left([x, z],\left(x^{k}, y L_{2}^{y} \cdots L_{j}^{y} L_{y x}, z^{m}\right) L_{x x}^{r-j}, x\right)^{+}\right. \\
& =-\left([x, z] L_{x x}^{r-j},\left(x^{k}, y L_{2}^{y} \cdots L_{j}^{y} L_{y x}, z^{m}\right), x\right)^{+} .
\end{aligned}
$$

Mas, pela equação (4.2), $[x, z] L_{x, x}^{r-j}=[u, v]-\frac{1}{2}(w, x, x)=[u, v]$, onde $u$ e $v$ dependem somente de $x$ e $z$. Daí, usando as equações (4.13), (4.7) e finalmente (4.13), junto com o teorema de Artin,

$$
\begin{aligned}
\left((y, x,[x, z])^{+} L_{2}^{y} \cdots L_{r}^{y},\left(x^{k}, y, z^{m}\right), x\right)^{+}= & -\left([u, v],\left(x^{k}, y L_{2}^{y} \cdots L_{j}^{y} L_{x, y}, z^{m}\right), x\right)^{+} \\
= & {[u, v] \circ\left(\left(x^{k}, y L_{2}^{y} \cdots L_{j}^{y} L_{x y}, z^{m}\right) \circ x\right) } \\
= & {[u, v] \circ\left\{\left(x^{k}, y L_{2}^{y} \cdots L_{j}^{y} L_{x y} \circ x, z^{m}\right)\right.} \\
& \left.-[u, v] \circ\left(x^{k}, x, z^{m}\right) \circ y L_{2}^{y} \cdots L_{j}^{y} L_{x y}\right\} \\
= & 0 .
\end{aligned}
$$

Finalmente, pelas equações $(4.28)_{d},(4.25)_{d-1}$ e $(4.24)_{d-1}$, temos que

$$
\left([y, z] L_{x, x} L_{2}^{y} \cdots L_{r}^{y},\left(x^{k}, y, z^{m}\right), x\right)^{+}=-\left([y, z],\left(x^{k}, y, z^{m}\right) L_{1}^{y} \cdots L_{r}^{y}, x\right)^{+} .
$$

Daí, por (4.29),

$$
\left([y, z],\left(x^{k}, y, z^{m}\right) L_{1} \cdots L_{r}, x\right)^{+}=\left([y, z],\left(x^{k}, y, z^{m}\right) L_{1} \cdots L_{r}, x\right)^{+}
$$

e, portanto, é zero.

Analisemos agora o caso em que $l=2$. Pela simetria entre $x$ e $z$ deste caso e por comodidade, trocamos $x \operatorname{com} z$.

Antes de seguir, provaremos a seguinte identidade:

$$
\left([x, y],\left(x^{k}, y, z^{m}\right) \circ y, z\right)^{+}=0
$$

Para demonstrá-la, dividimos em casos. Se $k=1$, é conseqüência direta de (4.19) com $l=2$ e a equação (4.7). Se $m=1$, substituímos em (4.19) 
$y=x, z=y, x=z, l=k$ e $k=1$, obtendo assim $\left([x, y],\left(x^{k}, y, z\right), z\right)^{+}=0$. Daí, $\left([x, y],\left(x^{k}, y, z\right), u\right)^{+}=-\left([x, y],\left(x^{k}, y, u\right), z\right)^{+}$. Portanto, usando (4.7) e o teorema de Artin, (4.11) e finalmente pelas equações (4.7), (4.13) e (4.19) juntas, temos que

$$
\begin{aligned}
\left([x, y],\left(x^{k}, y, z\right) \circ y, z\right)^{+} & =\left([x, y],\left(x^{k}, y, z \circ y\right), z\right)^{+} \\
& =-\left([x, y],\left(x^{k}, y, z\right), z \circ y\right)^{+} \\
& =-\left([x, y],\left(x^{k}, y, z\right), z\right)^{+} \circ y-\left([x, y],\left(x^{k}, y, z\right), y\right)^{+} \circ z \\
& =0 .
\end{aligned}
$$

Por último, se $k=m=2$, então pela equação (4.13),

$$
\begin{aligned}
\left([x, y],\left(x^{2}, y^{2}, z^{2}\right), z\right)^{+} & =\left([x, y] \circ\left(x^{2}, y^{2}, z^{2}\right)\right) \circ z-[x, y] \circ\left(\left(x^{2}, y^{2}, z^{2}\right) \circ z\right) \\
& =-[x, y] \circ\left(\left(x^{2}, y^{2}, z^{2}\right) \circ z\right) .
\end{aligned}
$$

Agora, usando (4.7) e (4.13), temos

$$
\begin{aligned}
\left([x, y],\left(x^{2}, y^{2}, z^{2}\right), z\right)^{+} & =\frac{2}{3}\left\{[x, y] \circ\left(x^{2}, y^{2}, z^{3}\right)+[x, y] \circ\left(\left(x^{2}, y^{2}, z\right) L_{z, z}\right)\right\} \\
& =\frac{2}{3}[x, y] \circ\left(x^{2}, y^{2}, z\right) L_{z, z} .
\end{aligned}
$$

Portanto, este caso se reduz ao $(4.26)_{d}$ com $m=1$.

Voltemos ao caso $l=2$ e apliquemos $(4.27)_{d-1}$ e $(4.23)_{d-1}$.

$$
\begin{aligned}
\left([x, y],\left(x^{k}, y^{2}, z^{m}\right) L_{1} \cdots L_{r}, z\right)^{+} & =2\left([x, y],\left(x^{k}, y, z^{m}\right) L_{1} \cdots L_{r} \circ y, z\right)^{+} \\
& =2\left([x, y], y \circ\left(x^{k}, y L_{1}^{y} \cdots L_{r}^{y}, z^{m}\right), z\right)^{+} .
\end{aligned}
$$

Usando agora a linearização parcial com respeito a $y$ da equação (4.30) e depois as equações $(4.26)_{d}$ e (4.7), temos que

$$
\begin{aligned}
2\left([x, y],\left(x^{k}, y L_{1}^{y} \cdots L_{r}^{y}, z^{m}\right) \circ y, z\right)^{+}= & -2\left(\left[x, y L_{1}^{y} \cdots L_{r}^{y}\right],\left(x^{k}, y, z^{m}\right) \circ y, z\right)^{+} \\
& -2\left([x, y],\left(x^{k}, y, z^{m}\right) \circ y L_{1}^{y} \cdots L_{r}^{y}, z\right)^{+} \\
= & -2\left(\left[x, y L_{1}^{y} \cdots L_{r}^{y}\right],\left(x^{k}, y^{2}, z^{m}\right), z\right)^{+} .
\end{aligned}
$$

A partir daqui, pelos mesmos argumentos do caso $l=1$, obtemos

$$
\left([x, y],\left(x^{k}, y^{2}, z^{m}\right) L_{1} \cdots L_{r}, z\right)^{+}=0 .
$$

Portanto, se $u=[y, z],(4.26)_{d}$ fica provada. 


\section{$4^{\circ}$ caso}

Se $y=(x, y, z)^{+}$, escrevamos por simplicidade $w=\left(x^{k}, y^{l}, z^{m}\right) L_{1} \cdots L_{r}$. Das equações $(4.28)_{d},(4.25)_{d-1}$ e $(4.23)_{d-2}$, segue:

$$
\begin{aligned}
\left((x, y, z)^{+}, w, x\right)^{+} & =-\left(z L_{y, x}, w, x\right)^{+} \\
& =-\left(z, w L_{y, x}, x\right)^{+}+\left(z, w, x L_{y, x}\right)^{+} \\
& =-\left(z, w L_{y, x}, x\right)^{+} \\
& =-\left(z,\left(x^{k}, y^{l}, z^{m}\right) L_{1} \cdots L_{j} L_{y, x} L_{x, x}^{r-j}, x\right)^{+} \\
& =-\left(z,\left(x^{k}, y^{l} L_{1} \cdots L_{j} L_{y, x}, z^{m}\right) L_{x, x}^{r-j}, x\right)^{+} .
\end{aligned}
$$

Notemos que da equação (4.7) e do teorema de Artin, segue

$$
\left(x^{k}, u, z^{m}\right) L_{x, x}=\left(x^{k}, u L_{x, x}, z^{m}\right) .
$$

Daí e pela equação (4.18), temos que

$$
\left((x, y, z)^{+}, w, x\right)^{+}=-\left(z,\left(x^{k}, y^{l} L_{1} \cdots L_{j} L_{y, x} L_{x, x}^{r-j}, z^{m}\right), x\right)^{+}=0 .
$$

\section{$5^{\circ}$ caso}

Se $u=(y, y, z)^{+}$, fazendo uso de (4.10) e (4.24) $d-1$, obtemos

$$
\begin{aligned}
((y, y, z), w, x)^{+} & =\left(y,(y, w, x)^{+}, z\right)^{+}-\left(y,(y, w, z)^{+}, x\right)^{+}-\left(y, w,(y, x, z)^{+}\right)^{+} \\
& =-\left(y, w,(y, x, z)^{+}\right)^{+}
\end{aligned}
$$

que se reduz ao caso anterior, trocando $x$ por $y$. Com isto, a proposição fica provada.

Lema 23 A seguinte identidade é satisfeita em $A_{3}$ :

$$
\left(x^{k}, y^{l}, z^{m}\right) L_{1} \cdots L_{r} L_{x,[y, z]}=-\left(x^{k}, y^{l}, z^{m}\right) L_{1} \cdots L_{r} L_{y,[x, z]} .
$$

Demonstração: Pela identidade (4.25), é claro que podemos supor $r=0$. Além disso, usando (4.9), (4.24) e (4.13), temos que

$$
\begin{aligned}
\left(\left(x^{k}, y^{l}, z^{m}\right), x,[y, z]\right)^{+} & =-\left(x,[y, z],\left(x^{k}, y^{l}, z^{m}\right)\right)^{+}-\left([y, z],\left(x^{k}, y^{l}, z^{m}\right), x\right)^{+} \\
& =\left(\left(x^{k}, y^{l}, z^{m}\right),[y, z], x\right)^{+} \\
& =\left(x^{k}, y^{l}, z^{m}\right) \circ([y, z] \circ x) .
\end{aligned}
$$


Aplicando agora a linearização parcial de (4.12) e depois (4.9), (4.13) e (4.24), obtemos

$$
\begin{aligned}
\left(\left(x^{k}, y^{l}, z^{m}\right), x,[y, z]\right)^{+} & =-\left(x^{k}, y^{l}, z^{m}\right) \circ([x, z] \circ y)+\left(x^{k}, y^{l}, z^{m}\right) \circ[x \circ y, z] \\
& =-\left(\left(x^{k}, y^{l}, z^{m}\right), y,[x, z]\right)^{+}+\left(x^{k}, y^{l}, z^{m}\right) \circ[x \circ y, z] .
\end{aligned}
$$

Pelo Corolário 2, $\left(x^{k}, u, z^{m}\right) \circ[u, z]=0$. Linearizando esta equação com respeito a $u$ e aplicando (4.13), temos

$$
\begin{aligned}
\left(x^{k}, y^{l}, z^{m}\right) \circ[x \circ y, z] & =-\left(x^{k}, y \circ x, z^{m}\right) \circ\left[y^{l}, z\right] \\
& =\left(x \circ\left(x^{k}, y, z^{m}\right)\right) \circ\left[y^{l}, z\right]+x \circ\left(\left(x^{k}, y, z^{m}\right) \circ\left[y^{l}, z\right]\right) \\
& =-\left(x,\left(x^{k}, y, z^{m}\right),\left[y^{l}, z\right]\right)^{+}
\end{aligned}
$$

Aplicando agora a linearização parcial de (4.19) com respeito a $y, \operatorname{com} l=1$, e depois (4.24), obtemos

$$
\left(x^{k}, y^{l}, z^{m}\right) \circ[x \circ y, z]=\left(x,\left(x^{k}, y^{l}, z^{m}\right),[y, z]\right)^{+}=0 .
$$

Daí, $\left(\left(x^{k}, y^{l}, z^{m}\right), x,[y, z]\right)^{+}=\left(\left(x^{k}, y^{l}, z^{m}\right), y,[x, z]\right)^{+}$, ou seja, o lema está provado.

\subsection{Prova do resultado principal}

Diremos que $f \in \operatorname{Alt}[X]$ é uma identidade fraca da álgebra $C$ se $f\left(a_{1}, \ldots, a_{n}\right)=$ 0 para elementos arbitrários $a_{1}, \ldots, a_{n} \in C_{7}^{(-)}$. Por exemplo, se $x \in C_{7}^{(-)}$, então $x^{2}=-n(x) \in \Phi$ e daí $f(x, y)=\left[x^{2}, y\right]=0$ é uma identidade fraca.

A idéia da prova está baseada em dois resultados que podem ser vistos em [12, pp.268-274]. O primeiro diz que o radical de Zhevlakov $\zeta(A)$ de uma álgebra alternativa $A$ coincide com o nil-radical da álgebra. O segundo diz que no caso da álgebra $A_{n}$ vale $\zeta\left(A_{n}\right)=T(C) \cap D\left(A_{n}\right)$. Usando identidades fracas e os operadores $L_{i}$, acharemos uma base $B$ de $D\left(A_{3}\right)$ como espaço vetorial sobre $\Phi$, além disso, provaremos que $T(C) \cap D\left(A_{3}\right)=(0)$ com o qual ficará provada a não existência de elementos nilpotentes em $A_{3}$.

Lema 24 Em Alt $[X]$ os polinômios $\left[x_{1}, x_{2}, x_{3}, x_{4}\right]$ e $\left[\left[x_{1}, x_{2}\right],\left[x_{3}, x_{4}\right]\right]$ pertencem ao espaço vetorial gerado pelos associadores de Jordan em $\left\{x_{i},\left[x_{i}, x_{j}\right]\right.$ : $i, j \in\{1, \ldots, 4\}\}$. 
Demonstração: Usando (4.2), temos que

$[x, y, z, x]=[[[x, y], z], x]=[x,[z,[x, y]]]=4(z, x,[x, y])^{+}+2(z, x,[x, y])$

e

$\left[(x, x, y)^{+}, z\right]=\frac{1}{4}[[x,[x, y]], z]=\frac{1}{4}[z,[[x, y], x]]=([x, y], z, x)^{+}+\frac{1}{2}([x, y], z, x)$.

Portanto, $2(z, x,[x, y])=4\left\{\left[(x, x, y)^{+}, z\right]-([x, y], z, x)^{+}\right\}$. Daí e da primeira equação, segue que

$$
[x, y, z, x]=4\left\{\left[(x, x, y)^{+}, z\right]-([x, y], z, x)^{+}-([x, y], x, z)^{+}\right\} .
$$

Por outro lado, pela equação (4.12) linearizada,

$$
\begin{aligned}
{\left[(x, x, y)^{+}, z\right]=} & {\left[x^{2} \circ y, z\right]-[x \circ(x \circ y), z] } \\
= & x^{2} \circ[y, z]+2 y \circ(x \circ[x, z])-x \circ[x \circ y, z]-(x \circ y) \circ[x, z] \\
= & x^{2} \circ[y, z]+y \circ(x \circ[x, z])-x \circ(x \circ[y, z]) \\
& -x \circ(y \circ[x, z])-(y, x,[x, z])^{+} \\
= & (x, x,[y, z])^{+}-2(y, x,[x, z])^{+}+(x, y,[x, z])^{+}+([x, z], x, y)^{+} .
\end{aligned}
$$

Substituindo esta equação em (4.32), obtemos

$$
\begin{aligned}
{[x, y, z, x]=} & 4\left\{2([x, z], x, y)^{+}-([x, z], y, x)^{+}-([y, z], x, x)^{+}\right. \\
& \left.-([x, y], z, x)^{+}-([x, y], x, z)^{+}\right\} .
\end{aligned}
$$

Agora, usando a equação (4.1), temos

$$
\begin{aligned}
{[(x, y, z), x] } & =\frac{1}{6}\{[[x, y, z], x]+[[y, z, x], x]+[[z, x, y], x]\} \\
& =\frac{1}{6}\{[x, y, z, x]+[y, z, x, x]-[x, z, y, x]\} .
\end{aligned}
$$

Por outro lado, aplicando (4.2),

$$
4(x, x,[y, z])^{+}=-2(x, x,[y, z])+[x,[x,[y, z]]]=[y, z, x, x] .
$$

Daí e aplicando (4.33) duas vezes, obtemos

$$
\begin{aligned}
{[(x, y, z), x]=} & \frac{2}{3}\left\{2([x, z], x, y)^{+}-([x, z], y, x)^{+}-([y, z], x, x)^{+}\right. \\
& -([x, y], z, x)^{+}-([x, y], x, z)^{+}+(x, x,[y, z])^{+} \\
& -2([x, y], x, z)^{+}+([x, y], z, x)^{+}+([z, y], x, x)^{+} \\
& \left.+([x, z], y, x)^{+}+([x, z], x, y)^{+}\right\} \\
= & \frac{2}{3}\left\{3(y, x,[z, x])^{+}+3([y, x], x, z)^{+}+3(x, x,[y, z])^{+}\right\} .
\end{aligned}
$$


Portanto, provamos que

$$
[(x, y, z), x]=2\left\{([x, z], x, y)^{+}+(z, x,[x, y])^{+}+(x, x,[y, z])^{+}\right\} .
$$

Além disso, pela equação (4.12),

$$
\begin{aligned}
{\left[(y, x, z)^{+}, x\right] } & =(y \circ x) \circ[z, x]+z \circ(x \circ[y, x]-(x \circ z) \circ[y, x]-y \circ(x \circ[z, x]) \\
& =(y, x,[z, x])^{+}-(z, x,[y, x])^{+} \\
& =([x, z], x, y)^{+}+(z, x,[x, y])^{+} .
\end{aligned}
$$

Substituindo esta equação em (4.34), obtemos

$$
[(x, y, z), x]=2\left\{\left[(y, x, z)^{+}, x\right]+(x, x,[y, z])^{+}\right\} .
$$

Para provar o lema, observemos que

$$
\left[\left[x_{1}, x_{2}\right],\left[x_{3}, x_{4}\right]\right]=\frac{1}{4 !} \sum_{\sigma \in S_{4}}(\operatorname{Sgn} \sigma)\left(\left[x_{\sigma(1)}, x_{\sigma(2)}\right],\left[x_{\sigma(3)}, x_{\sigma(4)}\right]\right)+\sum_{p} \alpha_{p} b_{p},
$$

onde cada $b_{p}$ é da forma $\left[\left[x_{i}, x_{k}\right],\left[x_{j}, x_{l}\right]\right]+\left[\left[x_{i}, x_{j}\right],\left[x_{k}, x_{l}\right]\right]$. Para isto é suficiente usar a anticomutatividade de [, ] e somar ou restar o $b_{p}$ adequado cada vez que $\sigma \in S_{4}$ seja tal que $\{\sigma(1), \sigma(2)\} \notin\{\{1,2\},\{3,4\}\}$. Por exemplo, quando na primeira soma considera-se $\sigma=(13)$, então

$$
\begin{aligned}
{\left[\left[x_{\sigma(1)}, x_{\sigma(2)}\right],\left[x_{\sigma(3)}, x_{\sigma(4)}\right]\right] } & =\left[\left[x_{3}, x_{2}\right],\left[x_{1}, x_{4}\right]\right] \\
& =\left[\left[x_{1}, x_{4}\right],\left[x_{2}, x_{3}\right]\right]
\end{aligned}
$$

e neste caso, o $b_{(13)}$ adequado é $b_{(13)}=-\left[\left[x_{1}, x_{4}\right],\left[x_{2}, x_{3}\right]\right]+\left[\left[x_{1}, x_{2}\right],\left[x_{4}, x_{3}\right]\right]$. Ao fazer a soma obtemos $\left[\left[x_{1}, x_{2}\right],\left[x_{4}, x_{3}\right]\right]=-\left[\left[x_{1}, x_{2}\right],\left[x_{3}, x_{4}\right]\right]$. Além disso, novamente pela anticomutatividade de $[$,$] , temos que a primeira somatória$ é zero. Isto decorre do fato de que, dado $\sigma \in S_{4}$, existe uma única $\tau \in S_{4}$ tal que $(\sigma(1), \sigma(2), \sigma(3), \sigma(4))=(\tau(3), \tau(4), \tau(1), \tau(2))$. É claro que o $\tau$ assim definido tem a mesma paridade de $\sigma$, além disso, $\left[\left[x_{\sigma(1)}, x_{\sigma(2)}\right],\left[x_{\sigma(3)}, x_{\sigma(4)}\right]\right]=$ - $\left[\left[x_{\tau(1)}, x_{\tau(2)}\right],\left[x_{\tau(3)}, x_{\tau(4)}\right]\right]$. Portanto, a somatória se divide em duas somas que se anulam entre si.

Falta provar que os $b_{p}$ têm a forma requerida. Mas, pela equação (4.2),

$$
\begin{aligned}
b_{p} & =\left[\left[x_{i}, x_{j}\right],\left[x_{k}, x_{l}\right]\right]+\left[\left[x_{i}, x_{k}\right],\left[x_{j}, x_{l}\right]\right] \\
& =4\left(x_{k},\left[x_{i}, x_{j}\right], x_{l}\right)^{+}+4\left(x_{j},\left[x_{i}, x_{k}\right], x_{l}\right)^{+}+2\left(x_{k},\left[x_{i}, x_{j}\right], x_{l}\right)+2\left(x_{j},\left[x_{i}, x_{k}\right], x_{l}\right) .
\end{aligned}
$$


Portanto, é suficiente verificar que a soma dos dois últimos termos pertence ao espaço vetorial gerado pelos associadores de Jordan em $\left\{x_{i},\left[x_{i}, x_{j}\right] \mid i, j \in\right.$ $\{1, \ldots, 4\}\}$, que chamaremos de $E$. Daqui em diante, para facilitar a notação, escreveremos $r \equiv s$ se $r-s \in E$.

Pela equação (4.2),

$$
2\left(x_{k},\left[x_{i}, x_{j}\right], x_{l}\right)=-2\left(\left[x_{i}, x_{j}\right], x_{k}, x_{l}\right) \equiv-\left[x_{k},\left[\left[x_{i}, x_{j}\right], x_{l}\right]\right]=-\left[x_{j}, x_{i}, x_{l}, x_{k}\right] .
$$

Analogamente, $2\left(x_{j},\left[x_{i}, x_{k}\right], x_{l}\right) \equiv-\left[x_{k}, x_{i}, x_{l}, x_{j}\right]$. Portanto, pela linearização parcial de (4.33) na variável $x$, é claro que $b_{p} \equiv-\left[x_{j}, x_{i}, x_{l}, x_{k}\right]-$ $\left[x_{k}, x_{i}, x_{l}, x_{j}\right] \in E$. Finalmente, aplicando (4.2),

$$
\begin{aligned}
{\left[x_{1}, x_{2}, x_{3}, x_{4}\right] } & =\left[\left[\left[x_{1}, x_{2}\right], x_{3}\right], x_{4}\right]=-\left[x_{4},\left[\left[x_{1}, x_{2}\right], x_{3}\right]\right] \\
& \equiv-2\left(\left[x, x_{2}\right], x_{4}, x_{3}\right)=2\left(x_{4},\left[x_{1}, x_{2}\right], x_{3}\right) \\
& =\left[\left[x_{1}, x_{2}\right],\left[x_{4}, x_{3}\right]\right] \in E
\end{aligned}
$$

Com o que fica provado o lema.

Lema 25 Sejam $\left\{k_{i}, l_{i}, m_{i} \mid i=1,2\right\} \subseteq\{1,2\}$. Se chamarmos de $w_{i}=\left(x^{k_{i}}, y^{l_{i}}, z^{m_{i}}\right) L_{1}^{(i)} \cdots L_{n_{i}}^{(i)}$, onde $L_{j}^{(i)} \in V, n_{i} \in \mathbb{N} \cup\{0\}$ e a,b $\in \Lambda$, então, em $A_{3}$, são satisfeitas as seguintes relações:

$$
\begin{aligned}
& {\left[w_{1} L_{a, b}, w_{2}\right]=\left[w_{1}, w_{2} L_{a, b}\right],} \\
& \left(w_{1} L_{a, b}, w_{2}, c\right)=\left(w_{1}, w_{2} L_{a, b}, c\right), \\
& \left(w_{1} L_{a, b}, c, w_{2}\right)^{+}=\left(w_{1}, c, w_{2} L_{a, b}\right)^{+} .
\end{aligned}
$$

Demonstração: Provemos primeiro que (4.38) segue de (4.36) e (4.37). Com efeito, aplicando (4.2) duas vezes e (4.36) e (4.37), obtemos

$$
\begin{aligned}
\left(w_{1} L_{a b}, c, w_{2}\right)^{+} & =\frac{1}{4}\left(-2\left(w_{1} L_{a, b}, c, w_{2}\right)+\left[c,\left[w L_{a, b}, w_{2}\right]\right]\right) \\
& =\frac{1}{4}\left(-2\left(w_{1}, c, w_{2} L_{a, b}\right)+\left[c,\left[w_{1}, w_{2} L_{a, b}\right]\right]\right) \\
& =\left(w_{1}, c, w_{2} L_{a, b}\right)^{+}
\end{aligned}
$$

Antes de provar (4.36), mostremos que $\left[b, w_{2} \circ\left(w_{1} \circ a\right)\right]=0, a \in \Lambda$. Basta considerar $a \in X_{3} \cup\left[X_{3}, X_{3}\right]$. Se $a \in\left[X_{3}, X_{3}\right]$, então, pela equação (4.26), $w_{1} \circ a=0$. Se $a \in X_{3}$, podemos supor $a=x$. Neste caso, se 
$w_{1}=\left(x^{k}, y^{l}, z^{m}\right) L_{1} \cdots L_{r}$ e $r \neq 0$, então, escrevendo $w_{1}=w L_{r}, L_{r}=L_{a, b} \mathrm{e}$ usando (4.20) e (4.26), temos

$w_{2} \circ\left(w_{1} \circ x\right)=w_{2} \circ\left(\left(w, a_{r}, b_{r}\right)^{+} \circ x\right)=w_{2} \circ\left[\left(x, b_{r}, a_{r}\right)^{+} \circ w+\left(w \circ x, b_{r}, a_{r}\right)^{+}\right]=0$.

Se $r=0$, pelo teorema de Artin e (4.7), $w_{2} \circ\left(\left(x^{k}, y^{l}, z^{m}\right) \circ x\right)=w_{2} \circ\left(x^{2}, y^{l}, z^{m} \circ\right.$ $x)$. Neste caso, se $w_{2}=\left(x^{k^{\prime}}, y^{l^{\prime}}, z^{m^{\prime}}\right) L_{1}^{\prime} \cdots L_{s}^{\prime}$ e $s \neq 0$, então, das equações (4.2) e (4.26), $w_{2} \circ\left(x^{k}, y^{l}, z^{m} \circ x\right)=0$. Por último, se $s=0$, de (4.2), (4.24) e (4.14), segue que

$$
\begin{aligned}
w_{2} \circ\left(w_{1} \circ a\right) & =\left(x^{k^{\prime}}, y^{l^{\prime}}, z^{m^{\prime}}\right) \circ\left(x^{k}, y^{l}, z^{m} \circ x\right) \\
& =\left(x^{k^{\prime}}, y^{l^{\prime}}, z^{m^{\prime}}\right) \circ\left\{-2\left(x^{k}, y^{l}, z^{m} \circ x\right)^{+}+\frac{1}{2}\left[y^{l},\left[x^{k}, z^{m} \circ x, x\right]\right]\right\} \\
& =\left(x^{k^{\prime}}, y^{l^{\prime}}, z^{m^{\prime}}\right) \circ\left[y^{l},\left[x^{k}, z^{m} \circ x\right]\right] \in Z(A) .
\end{aligned}
$$

Portanto, em qualquer caso, $\left[b, w_{2} \circ\left(w_{1} \circ x\right)\right]=0$.

Provemos agora (4.36).

$$
\left[\left(w_{1}, a, b\right)^{+}, w_{2}\right]=\left[\left(w_{1} \circ a\right) \circ b, w_{2}\right]-\left[w_{1} \circ(a \circ b), w_{2}\right] .
$$

Mas, pela equação (4.2),

$$
\begin{aligned}
{\left[\left(w_{1} \circ a\right) \circ b, w_{2}\right] } & =\left(w_{1} \circ a\right) \circ\left[b, w_{2}\right]+b \circ\left[w_{1} \circ a, w_{2}\right] \\
& =\left[b, w_{2} \circ\left(w_{1} \circ a\right)\right]-w_{2} \circ\left[b, w_{1} \circ a\right]+b \circ\left[w_{1} \circ a, w_{2}\right] \\
& =w_{2} \circ\left[b, w_{1} \circ a\right]+b \circ\left[w_{1} \circ a, w_{2}\right]
\end{aligned}
$$

e usando (4.12), temos que

$$
\begin{aligned}
{\left[b, w_{1} \circ a\right] \circ w_{2} } & =\left[w_{2} \circ b, w_{1} \circ a\right]-b \circ\left[w_{2}, w_{1} \circ a\right] \\
& =\left[b \circ w_{2}, w_{1} \circ a\right]+\left[w_{1} \circ a, w_{2}\right] \circ b
\end{aligned}
$$

e $\left[w_{1} \circ a, w_{2}\right]=\left[w_{1}, w_{2}\right] \circ a+\left[a, w_{2}\right] \circ w_{1}=\left[w_{1}, w_{2} \circ a\right]-\left[w_{1} \circ w_{2}, a\right]$. Mas, pelo já visto, $w_{1} \circ w_{2} \in Z(A)$ e, daí, $\left[w_{1} \circ a, w_{2}\right]=\left[w_{1}, w_{2} \circ a\right]$, de onde segue que

$$
\left[b, w_{1} \circ a\right] \circ w_{2}=\left[b \circ w_{2}, w_{1} \circ a\right]+\left[w_{1}, w_{2} \circ a\right] \circ b .
$$

Por outro lado, usando novamente (4.12), $\left[b \circ w_{2}, w_{1} \circ a\right]=\left[b \circ w_{2}, w_{1}\right] \circ a+\left[w_{2} \circ\right.$ $b, a] \circ w_{1}$. Mas, $\left[b \circ w_{2}, a\right] \circ w_{1}=-\left[w_{1}, a\right] \circ\left[b \circ w_{2}\right]={ }^{\prime}-\left[w_{1}, a \circ\left(b \circ w_{2}\right)\right]+\left[w_{1}, b \circ w_{2}\right] \circ$ 
a. Portanto, pelas duas últimas equações, $\left[b \circ w_{2}, w_{1} \circ a\right]=-\left[w_{1}, a \circ\left(b \circ w_{2}\right)\right]$, de onde segue que

$$
\begin{aligned}
{\left[b, w_{1} \circ a\right] \circ w_{2} } & =\left[b \circ w_{2}, w_{1} \circ a\right]-\left[w_{2}, w_{1} \circ a\right] \circ b \\
& =-\left[w_{1}, a \circ\left(b \circ w_{2}\right)\right]+\left[w_{1} \circ a, w_{2}\right] \circ b
\end{aligned}
$$

Usando estas igualdades, obtemos

$$
\begin{aligned}
{\left[\left(w_{1} \circ a\right) \circ b, w_{2}\right] } & =-\left[w_{2},\left(w_{1} \circ a\right) \circ b\right]+\left[w_{2} \circ\left(w_{1} \circ a\right), b\right] \\
& =-\left[w_{2},\left(w_{1} \circ a\right) \circ b\right]+\left(w_{1} \circ a\right) \circ\left[w_{2}, b\right]+w_{2} \circ\left[w_{1} \circ a, b\right] \\
& =-b \circ\left[w_{2}, w_{1} \circ a\right]-w_{2} \circ\left[b, w_{1} \circ a\right],
\end{aligned}
$$

de onde segue que $\left[\left(w_{1} \circ a\right) \circ b, w_{2}\right]=-\left[\left(w_{2} \circ b\right) \circ a, w_{1}\right]=\left[w_{1},\left(w_{2} \circ b\right) \circ a\right]$. Portanto, $\left[w_{1},\left(w_{2} \circ b\right) \circ a\right]=\left[\left(w_{1} \circ a\right) \circ b, w_{2}\right]$. Finalmente, usando (4.12) várias vezes e (4.16), temos que

$$
\begin{aligned}
{\left[w_{1} \circ(a \circ b), w_{2}\right] } & =\left[w_{1}, w_{2}\right] \circ(a \circ b)+\left[a \circ b, w_{2}\right] \circ w_{1} \\
& =\left[w_{1}, w_{2}\right] \circ(a \circ b)-\left[a \circ b, w_{1}\right] \circ w_{2}+\left[a \circ b, w_{1} \circ w_{2}\right] \\
& =\left[w_{1}, w_{2}\right] \circ(a \circ b)+\left[w_{1}, a \circ b\right] \circ w_{2} \\
& =\left[w_{1}, w_{2} \circ(a \circ b)\right] .
\end{aligned}
$$

Daí, obtemos que

$$
\begin{aligned}
{\left[\left(w_{1}, a, b\right)^{+}, w_{2}\right] } & =\left[\left(w_{1} \circ a\right) \circ b, w_{2}\right]-\left[w_{1} \circ(a \circ b), w_{2}\right] \\
& =\left[w_{1},\left(w_{2} \circ b\right) \circ a\right]-\left[w_{1}, w_{2} \circ(a \circ b)\right] \\
& =\left[w_{1},\left(w_{2}, b, a\right)^{+}\right] .
\end{aligned}
$$

Finalmente, de (4.9) e (4.24), temos que $\left(w_{2}, b, a\right)^{+}=\left(w_{2}, a, b\right)^{+}$. Portanto, (4.36) está provada. A equação (4.37) é análoga. (4.36),

Nas mesmas condições do lema, se $k_{1}=k_{2}, l_{1}=l_{2}$ e $m_{1}=m_{2}$, então, por

$$
\begin{aligned}
{\left[w_{1}, w_{2}\right] } & =\left[\left(x^{k}, y^{l}, z^{m}\right) L_{1}^{(1)} \cdots L_{r_{1}}^{(1)},\left(x^{k}, y^{l}, z^{m}\right) L_{1}^{(2)} \cdots L_{r_{2}}^{(2)}\right] \\
& =\left[\left(x^{k}, y^{l}, z^{m}\right),\left(x^{k}, y^{l}, z^{m}\right) L_{1}^{(2)} \cdots L_{r_{2}}^{(2)} L_{r_{1}}^{(1)} \cdots L_{1}^{(1)}\right]
\end{aligned}
$$

Mas, de (4.25), segue que

$$
\begin{aligned}
{\left[w_{1}, w_{2}\right] } & =\left[\left(x^{k}, y^{l}, z^{m}\right) L_{1}^{(1)} \cdots L_{r_{1}}^{(1)} L_{1}^{(2)} \cdots L_{r_{2}}^{(2)},\left(x^{k}, y^{l}, z^{m}\right)\right] \\
& =-\left[\left(x^{k}, y^{l}, z^{m}\right),\left(x^{k}, y^{l}, z^{m}\right) L_{1}^{(1)} \cdots L_{r_{1}}^{(1)} L_{1}^{(2)} \cdots L_{r_{2}}^{(2)}\right] \\
& =-\left[w_{1}, w_{2}\right],
\end{aligned}
$$


de onde segue

$$
\left[w_{1}, w_{2}\right]=0
$$

Analogamente,

$$
\begin{aligned}
& \left(w_{1}, w_{2}, c\right)=0 \\
& \left(w_{1}, c, w_{2}\right)^{+}=0 .
\end{aligned}
$$

Por comodidade, introduzamos a seguinte notação: chamaremos $L_{1}=$ $L_{x, y}, L_{2}=L_{y, z}, L_{3}=L_{z, x}, L_{4}=L_{x, x}, L_{5}=L_{y, y}, L_{6}=L_{z, z}, L_{7}=L_{x,[y, z]} \mathrm{e}$ denotaremos por

$$
\begin{aligned}
W_{0} & :=\left\{(x, y, z) L_{1}^{r_{1}} \cdots L_{7}^{r_{7}}: r_{i} \in \mathbb{N} \cup\{0\}\right\}, \\
W_{1}:=\left\{[w, a]: w \in W_{0} \text { e } a \in X_{3}\right\} & \\
W_{2}:=\left\{(w, x, y),(w, y, z),(w, x, z): w \in W_{0}\right\} & \\
W & =W_{0} \cup W_{1} \cup W_{2},
\end{aligned}
$$

Proposição 5 Se chamarmos $D_{0}:=D \cap M\left[X_{3}\right]$, onde $D$ é o ideal gerado pelos associadores em $A$, então $D_{0}=\operatorname{Vect}_{\Phi} W$.

Demonstração: Para começar, notemos que como em $M\left[X_{3}\right]$ o produto definido é $a \cdot b=[a, b]$, temos que $D_{0}=\left\{\left[\left(u_{1}, u_{2}, u_{3}\right), u_{4}, \ldots, u_{r}\right]: u_{1}, \ldots, u_{r} \in\right.$ $\left.M\left[X_{3}\right]\right\}$. Com efeito, se chamarmos $I$ ao lado direito desta igualdade, é claro que $I \subseteq D_{0}$. Inversamente, $I$ é um ideal em $M\left[X_{3}\right]$. Além disso pela equação (4.2) temos que $[x,[y, z]]+[y,[z, x]]+[z,[x, y]]=6(x, y, z) \in I$ portanto em $M\left[X_{3}\right] / I$ se satisfaz $J(x, y, z)=0$, sendo assim uma álgebra de Lie sobre $\Phi$. Por outro lado, provemos que $M\left[X_{3}\right] / D_{0}$ é uma álgebra de Lie livre com geradores $\{\bar{x}, \bar{y}, \bar{z}\}$. Para isto é suficiente provar que para toda álgebra associativa $B$ existe um homomorfismo $\phi^{(-)}: M\left[X_{3}\right] / D_{0} \longrightarrow B^{(-)}$, onde $B^{(-)}$representa a álgebra obtida a partir de $B$ mudando a operação usual por [,], tal que $\phi^{(-)}(x)=a, \phi^{(-)}(y)=b$ e $\phi^{(-)}(z)=c$ para quaisquer $a, b$ e $c \in B$ (ver [7, p.57]). Para isto, notemos que $A_{3} / D \cong A s s\left(X_{3}\right)$, portanto dados $a, b$ e $c \in B$ existe um homomorfismo $\varphi: A / D \longrightarrow B$, tal que $\varphi(x)=a, \varphi(y)=b$ e $\varphi(z)=c$. Seja $\varphi^{(-)}:(A / D)^{(-)} \longrightarrow B^{(-)}$, definida por $\varphi^{(-)}([p, q])=[\varphi(p), \varphi(q)]$. É claro que $\varphi^{(-)}$é um homomorfismo. Por outro lado, considerando $A$ com o produto [,] temos que pelo $1^{o}$ Teorema de isomorfismo

$$
M\left[X_{3}\right] / D_{0} \cong\left(M\left[X_{3}\right]+D^{(-)}\right) / D^{(-)} \hookrightarrow A^{(-)} / D^{(-)} .
$$


Mas se definimos $\psi: A^{(-)} / D^{(-)} \longrightarrow(A / D)^{(-)}$por $\psi\left(a+D^{(-)}\right)=\bar{a}$ então $\psi$ é um isomorfismo, pelo que podemos considerar $M\left[X_{3}\right] / D_{0} \hookrightarrow(A / D)^{(-)}$ e desta forma, tomando $\phi=\left.\varphi\right|_{M\left[X_{3}\right] / D_{0}}$ obtemos o que queriamos. Agora bem, estendendo a um homomorfismo $i$ tal que $i(\bar{x})=\bar{x}, i(\bar{y})=\bar{y}$ e $i(\bar{z})=\bar{z}$ concluímos que $D_{0} \subseteq I$ e portanto $D_{0}=I$.

Para provar a inclusão $D_{0} \subseteq \operatorname{Vect}_{\Phi} W$, consideremos o polinômio $f=$ $\left[\left(u_{1}, u_{2}, u_{3}\right), u_{4}, \ldots, u_{r}\right]$, onde $u_{1}, \ldots, u_{r} \in M\left[X_{3}\right]$ e prossigamos por indução sobre $d(f)$. se $d(f)=3$, então $f=\alpha(x, y, z) \in \operatorname{Vect}_{\Phi} W_{0}$. Se $d(f) \geq 4$, mostraremos que podemos supor $r \geq 4$. Com efeito, se $d(f)=4$ e $r=3$, podemos supor $u_{1}=[y, x], u-2=x, u_{3}=z$ e, neste caso, pela equação (4.6), podemos transformar $f=([y, x], x, z)=[(x, y, z), x]$. Se $d(f) \geq 5$ e $r=3$, há dois casos para analisar.

$1^{\circ}$ caso: $d\left(u_{1}\right) \geq 2$ e $d\left(u_{2}\right) \geq 2$. Escrevamos $u_{1}=\left[u_{1}^{\prime}, u_{1}^{\prime \prime}\right]$ e $u_{2}=\left[u_{2}^{\prime}, u_{2}^{\prime \prime}\right]$. Aplicando a linearização parcial de (4.6) com respeito à variável $x$, obtemos

$$
f=\left(u_{1}, u_{2}, u_{3}\right)=\left[u_{2},\left(u_{1}^{\prime}, u_{1}^{\prime \prime}, u_{3}\right)\right]+\left[u_{1},\left(u_{1}^{\prime}, u_{2}, u_{3}\right)\right]-\left(\left[u_{1}^{\prime}, u_{2}\right], u_{1}^{\prime \prime}, u_{3}\right),
$$

onde os dois primeiros termos do lado direito são como queremos. Notemos que pela equação (4.1) podemos levar as expressões do tipo $((a, b, c), d, e)$ à forma requerida. Agora, pela equação (4.2), temos que

$$
\begin{aligned}
\left(\left[u_{1}^{\prime}, u_{2}\right], u_{1}^{\prime \prime}, u_{3}\right) & =\left(\left[u_{1}^{\prime},\left[u_{2}^{\prime}, u_{2}^{\prime \prime}\right]\right], u_{1}^{\prime \prime}, u_{3}\right) \\
& =4\left(\left(u_{2}^{\prime}, u_{1}^{\prime}, u_{2}^{\prime \prime}\right)^{+}, u_{1}^{\prime \prime}, u_{3}\right)+\left(\left(u_{2}^{\prime}, u_{1}^{\prime}, u_{2}^{\prime \prime}\right), u_{1}^{\prime \prime}, u_{3}\right) .
\end{aligned}
$$

Mas, usando (4.7) e (4.9), obtemos

$$
\begin{aligned}
\left(\left(u_{2}^{\prime}, u_{1}^{\prime}, u_{2}^{\prime \prime}\right)^{+}, u_{1}^{\prime \prime}, u_{3}\right)= & \left(u_{2}^{\prime}, u_{1}^{\prime},\left(u_{2}^{\prime \prime}, u_{1}^{\prime \prime}, u_{3}\right)\right)^{+}+\left(\left(u_{2}^{\prime}, u_{1}^{\prime}, u_{3}\right), u_{1}^{\prime \prime}, u_{2}^{\prime \prime}\right)^{+} \\
& +\left(u_{2}^{\prime},\left(u_{1}^{\prime}, u_{1}^{\prime \prime}, u_{3}\right), u_{2}^{\prime \prime}\right)^{+} \\
= & -\left(\left(u_{2}^{\prime \prime}, u_{1}^{\prime \prime}, u_{3}\right), u_{1}^{\prime}, u_{2}^{\prime}\right)^{+}+\left(\left(u_{2}^{\prime}, u_{1}^{\prime}, u_{3}\right), u_{1}^{\prime \prime}, u_{2}^{\prime \prime}\right)^{+} \\
& +\left(\left(u_{1}^{\prime}, u_{1}^{\prime \prime}, u_{3}\right), u_{2}^{\prime \prime}, u_{2}^{\prime}\right)^{+}-\left(\left(u_{1}^{\prime}, u_{1}^{\prime \prime}, u_{3}\right), u_{2}^{\prime}, u_{2}^{\prime \prime}\right)^{+} .
\end{aligned}
$$

Finalmente, pela equação (4.2) e a observação feita anteriormente, temos que todo elemento da forma $((a, b, c), d, e)^{+}$pode ser levado a uma soma de termos da forma $\left[\left(u_{1}, u_{2}, u_{3}\right), u_{4}, \ldots, u_{r}\right]$, com $r \geq 4$.

$2^{\circ}$ caso: Suponhamos que $d\left(u_{1}\right) \geq 3$. Neste caso, pela equação (4.2) e as observações feitas no primeiro caso, podemos supor $u_{1}=(a, b, c)^{+}$, onde $a, b, c \in M\left[X_{3}\right]$ e usando novamente (4.7), obtemos

$$
\left(u_{1}, u_{2}, u_{3}\right)=\left(\left(a, u_{2}, u_{3}\right), b, c\right)^{+}+\left(a,\left(b, u_{2}, u_{3}\right), c\right)^{+}+\left(a, b,\left(c, u_{2}, u_{3}\right)\right)^{+},
$$


caso que já foi discutido. Portanto, podemos supor $r \geq 4$.

Por outro lado, em toda álgebra de Maltsev é satisfeita a seguinte identidade (ver [8, p.429]):

$$
R_{y z, x}=R_{z} R_{x y}-R_{z x} R_{y}+\left[R_{y z}, R_{x}\right]
$$

pelo que podemos supor que $d\left(u_{r}\right) \leq 2$ e $f=\left[f_{1}, u_{r}\right]$, onde $f_{1} \in W$. Vamos analisar todos os casos possíveis.

a) $f_{1} \in W_{0}$ e $u_{r} \in X_{3}$. Então $f=\left[f_{1}, u_{r}\right] \in W_{1}$.

b) $f_{1} \in W_{0}$ e $u_{r} \in\left[X_{3}, X_{3}\right]$. Então, podemos supor $u_{r}=[x, y]$. Aplicando as equações (4.2), (4.26) e (4.9), obtemos

$$
\begin{aligned}
f & =\left[f_{1},[x, y]\right]=4(x, f, y)^{+}+2\left(x, f_{1}, y\right) \\
& =4\left(f_{1}, y, x\right)^{+}-4\left(f_{1}, x, y\right)^{+}+2\left(f_{1}, y, x\right) \in \operatorname{Vect}_{\Phi} W .
\end{aligned}
$$

c) Se $f_{1} \in W_{1}$ e $u_{r} \in X_{3}$, escrevamos $f_{1}=[w, a], w \in W_{0}, a \in X_{3}$. Neste caso, aplicando (4.2) e depois (4.26), obtemos

$$
\begin{aligned}
f & =\left[[w, a], u_{r}\right]=-\left[u_{r},[w, a]\right] \\
& =4\left(w, u_{r}, a\right)^{+}+2\left(w, u_{r}, a\right) \in \operatorname{Vect}_{\Phi}\left(W_{0} \cup W_{2}\right) .
\end{aligned}
$$

d) Se $f_{1} \in W_{1}$ e $u_{r} \in\left[X_{3}, X_{3}\right]$, podemos escrever $f=[[w, a],[b, c]], a, b, c \in$ $X_{3}$. Pelo Lema 24, substituindo $w=x_{1}, a=x_{2}, b=x_{3}$ e $c=x_{4}$, temos que $f=\left[f_{1}, u_{r}\right]$ pertence ao espaço vetorial gerado pelos associadores de Jordan em $\left\{x_{i},\left[x_{j}, x_{k}\right]: i, j, k \in\{1, \ldots, 4\}\right\}$. Portanto, é suficiente considerar os casos $f=(w, a,[b, c])^{+}$e $f=([w, a], b, c)^{+}$, onde $a, b, c \in$ $X_{3}$ e $w \in W_{0}$.

No primeiro caso, se $\{a, b, c\} \neq X_{3}$, podemos supor $a=b$ e neste caso, usando (4.24), (4.26) e (4.12), temos que

$$
\begin{aligned}
f=(w, b,[b, c])^{+} & =(w,[b, c], b)^{+} \\
& =-w \circ([b, c] \circ b)=-w \circ\left[b^{2}, c\right] \\
& =0 .
\end{aligned}
$$

Por outro lado, se $\{a, b, c\}=X_{3}$, então, usando (4.30) e a anticomutatividade de $[$,$] , temos f= \pm w L_{x,[y, z]}$, isto é, $f \in W_{0}$. 
No segundo caso, pela equação (4.12),

$$
([w, a], b, c)^{+}=-(w,[b, a], c)^{+}-(w, b,[c, a])^{+}+\left[(w, b, c)^{+}, a\right] .
$$

Como podemos ver, o último termo pertence a $W_{2}$ e os dois primeiros são como no caso anterior.

e) Se $f_{1} \in W_{2}$ e $u_{r} \in X_{3}$, então $f=[(w, a, b), c]$, onde $a, b, c \in X_{3}$ e neste caso, pela equação (4.2), temos $f=-2\left[(w, a, b)^{+}, c\right]+\frac{1}{2}[[a,[w, b]], c]$. O primeiro termo pertence a $W_{1}$ e $[[a,[w, b]], c]=-[w, b, a, c]$ se reduz, pelo Lema 4.6, aos casos analisados no item d).

f) Se $f \in W_{2}$ e $u_{r}=[c, d]$, podemos escrever $f_{1}=(w, a, b)$, com $w \in W_{0}$, $a, b, c, d \in X_{3}$. Como $\{a, b\} \cap\{c, d\} \neq \emptyset$, podemos supor $f_{1}=(w, x, a)$ e $u_{r}=[x, b]$. Substituindo $a=b=x$ em (4.22), obtemos

$$
\left(x^{2}, c, d\right)^{+}=2 x \circ(x, c, d)^{+}+\frac{1}{2}([c, x] \circ[x, d]),
$$

colocando $c=a$ e $d=b$, obtemos

$$
[x, b] \circ[x, a]=-2\left(x^{2}, a, b\right)^{+}+4 x \circ(x, a, b)^{+} .
$$

Agora, pela linearização parcial de (4.35), temos que

$$
\begin{aligned}
f= & {[(w, x, a),[x, b]] } \\
= & -[([x, b], x, a), w]+2\left\{\left[(x, w, a)^{+},[x, b]\right]+\left[(x,[x, b], a)^{+}, w\right]\right. \\
& \left.+(w,[x, b],[x, a])^{+}+([x, b], w,[x, a])^{+}\right\} .
\end{aligned}
$$

Aplicando (4.24) e (4.26), obtemos

$$
f=-2\left\{[([x, b], x, a), w]+\left[(x,[x, b], a)^{+}, w\right]+(w,[x, b],[x, a])^{+}\right\} .
$$

A seguir provaremos que cada um destes termos pertence a $\operatorname{Vect}_{\Phi} W$.

Comecemos pelo último. Aplicando (4.26), (4.11) e (4.42), temos que

$$
\begin{aligned}
(w,[x, b],[x, a])^{+} & =-w \circ([x, b] \circ[x, a]) \\
& =2 w \circ\left(x^{2}, a, b\right)^{+}-4\left(w,(x, a, b)^{+}, x\right)^{+} .
\end{aligned}
$$

Mas, por (4.11), segue que

$$
\begin{aligned}
\left(w,(x, a, b)^{+}, x\right)^{+}= & \left((w, x, x)^{+}, a, b\right)^{+}-\left(b,(w, a, x)^{+}, x\right)^{+} \\
& -\left((w, b, x)^{+}, a, x\right)^{+} \in \operatorname{Vect}_{\Phi} W_{0}
\end{aligned}
$$


e da equação (4.20), temos que

$$
w \circ\left(x^{2}, a, b\right)^{+}=-\left(x^{2} \circ w, b, a\right)^{+}+x^{2} \circ(w, b, a)^{+} .
$$

Denotando $a \equiv b$ se $a-b \in \operatorname{Vect}_{\Phi} W$, aplicando (4.20) e (4.26), temos que

$$
\begin{aligned}
\left(w \circ x^{2}, b, a\right)^{+} & =-\left((w, x, x)^{+}, b, a\right)^{+}-((w \circ x) \circ x, b, a)^{+} \\
& \equiv-((w \circ x) \circ x, b, a)^{+} \\
& =-(w \circ x) \circ(x, b, a)^{+}+x \circ(w \circ x, b, a)^{+} \\
& =-\left(x, w,(x, a, b)^{+}\right)^{+}+x \circ(w \circ x, b, a)^{+} \\
& \equiv x \circ(w \circ x, b, a)^{+} .
\end{aligned}
$$

Aplicando novamente (4.20) e depois (4.26), temos que

$$
\begin{aligned}
\left(w \circ x^{2}, b, a\right)^{+} & =-x \circ\left(w \circ(x, a, b)^{+}+x \circ\left(x \circ(w, b, a)^{+}\right)\right. \\
& =x \circ\left(x \circ(w, b, a)^{+}\right) \\
& =-\left(x, x,(w, b, a)^{+}\right)^{+}+x^{2} \circ(w, b, a)^{+} .
\end{aligned}
$$

Substituindo em (4.45), obtemos

$$
w \circ\left(x^{2}, a, b\right)^{+} \equiv\left((w, b, a)^{+}, x, x\right)^{+} .
$$

Portanto, $w \circ\left(x^{2}, a, b\right)^{+} \in \operatorname{Vect}_{\Phi} W$ e, daí, o terceiro termo de (4.43) pertence a $\operatorname{Vect}_{\Phi} W$, como queríamos provar.

Analisemos agora o segundo termo de (4.43). Aplicando várias vezes a linearização parcial de (4.12), temos

$$
\begin{aligned}
{\left[(x,[x, b], a)^{+}, w\right]=} & -[x \circ([x, b] \circ a), w]+[(x \circ[x, b]) \circ a, w] \\
= & -[x, w] \circ([x, b] \circ a)-[[x, b] \circ a, w] \circ x \\
& +[x \circ[x, b], w] \circ a+[a, w] \circ(x \circ[x, b]) \\
= & {[a, w] \circ(x \circ[x, b])+([x, w] \circ[x, w]) \circ a } \\
& +([[x, b], w] \circ x) \circ a-[x, w] \circ([x, b] \circ a) \\
& -([[x, b], w] \circ a) \circ x-([a, w] \circ[x, b]) \circ x .
\end{aligned}
$$

Portanto, depois de reagrupar, obtemos

$$
\begin{aligned}
{\left[(x,[x, b], a)^{+}, w\right]=} & ([x, w],[x, b], a)^{+}+(x,[[x, b], w], a)^{+} \\
& +(x,[x, b],[w, a])^{+} .
\end{aligned}
$$


A seguir, provamos que cada um dos termos do lado direito de (4.47) pertence a $\operatorname{Vect}_{\Phi} W$. Usando a linearização parcial de (4.12), como foi feito com segundo termo de (4.43), obtemos

$$
\begin{aligned}
{\left[(w,[x, b], a)^{+}, x\right]=} & ([w, x],[x, b], a)^{+}+(w,[x, b, x], a)^{+} \\
& +(w,[x, b],[a, x])^{+}
\end{aligned}
$$

de onde segue que

$$
\begin{aligned}
{[(w, x],[x, b], a)^{+}=} & -(w,[x, b, x], a)^{+}-(w,[x, b],[a, x])^{+} \\
& +\left[(w,[x, b], a)^{+}, x\right] .
\end{aligned}
$$

É claro, pela equação (4.30), que $\left[(w,[x, b], a)^{+}, x\right] \in \operatorname{Vect}_{\Phi} W_{1}$, e da equação (4.2) segue que $(w,[x, b, x], a)^{+}=\left(w,(b, x, x)^{+}, a\right)^{+}$. Daí, usando (4.11) como em (4.44), concluímos que $(w,[x, b, x], a)^{+} \in \operatorname{Vect}_{\Phi} W$. Finalmente, $(w,[x, b],[a, x])^{+}$é como o terceiro termo de (4.42) que, como já foi provado, pertence a $\operatorname{Vect}_{\Phi} W$. Portanto, provamos que o primeiro termo de (4.47) pertence a $\operatorname{Vect}_{\Phi} W$.

Analisemos agora o segundo termo de (4.47). Aplicando novamente a linearização parcial de (4.12) e (4.24) e os resultados obtidos até agora, temos

$$
\begin{aligned}
(x,[w,[x, b]], a)^{+} & =-(x, w,[a,[x, b]])^{+}-([x,[x, b]], w, a)^{+}+\left[(x, w, a)^{+},[x, b]\right] \\
& \equiv-(x, w,[a,[x, b]])^{+} .
\end{aligned}
$$

Usando (4.2), (4.11) e (4.9), temos $(x, w,[a,[x, b]])^{+} \equiv-2(x, w,(x, a, b))^{+}$ $=2(w,(x, a, b), x)^{+}+2((x, a, b), x, w)^{+}$. Notemos que $(x, a, b) \neq 0$ se $\mathrm{e}$ somente se $\{a, b\}=\{y, z\}$. Portanto, podemos supor $a=y, b=z$ e aplicar (4.41), obtendo assim $(x, w,[a,[x, b]]) \equiv 2(w,(x, a, b), x)^{+}$. Aplicando várias vezes a equação (4.7) e observando as inclusões já provadas, temos que

$$
\begin{aligned}
-(w,(x, a, b), x)^{+} & =(w, x,(x, a, b))^{+}+((a, w, b), x, x)^{+}+\left(b,(x, x, w)^{+}, a\right) \\
& \equiv-\left(a,(w, x, x)^{+}, b\right) \in \operatorname{Vect}_{\Phi} W .
\end{aligned}
$$

O terceiro termo de (4.47) á análogo ao primeiro de (4.47), que já foi discutido. Portanto, fica provado que o segundo termo de (4.42) pertence a $\operatorname{Vect}_{\Phi} W$. 
Por último, aplicando a linearização parcial de (4.32), (4.26), (4.2) e o argumento anterior, temos que

$$
\begin{aligned}
{[([x, b], x, a), w]=} & -[(w, x, a),[x, b]]+2\left[(x,[x, b], a)^{+}, w\right] \\
& +2\left(\left[(x, w, a)^{+},[x, b]\right]\right)+([x, b], w,[x, a])^{+} \\
& +(w,[x, b],[x, a])^{+} \\
\equiv & {[(w, x, a), x, b] . }
\end{aligned}
$$

Mas, pelas equações (4.6), (4.2) e (4.7), temos que

$$
\begin{aligned}
{[(w, x, a), x, b] } & =-[(w, x,[a, x]), b] \\
& =-\left(2\left[(w, x,[a, x])^{+}, b\right]+\frac{1}{2}[[w,[x, a, x], b])\right. \\
& \equiv-\frac{1}{2}\left[\left[w,(a, x, x)^{+}\right], b\right] \\
& =-\frac{1}{2}\left(\left[([w, a], x, x)^{+}, b\right]+\left[(a,[w, x], x)^{+}, b\right]+\left[(a, x,[w, x])^{+}, b\right]\right) .
\end{aligned}
$$

Portanto, é suficiente provar que $\left[b,([w, a], x, x)^{+}\right] \in \operatorname{Vect}_{\Phi} W$, já que os outros casos são tratados de forma análoga. Aplicando (4.7), obtemos:

$\left[b,([w, a], x, x)^{+}\right]=([b,[w, a]], x, x)^{+}+([w, a],[b, x], x)^{+}+([w, a], x,[b, x])^{+}$.

Os dois últimos termos são como o primeiro e o terceiro termos de (4.47). Para o primeiro termo, usemos (4.2), (4.7) e (4.9),

$$
\begin{aligned}
([b,[w, a]], x, x)^{+} & =4\left((w, a, b)^{+}, x, x\right)^{+}+2((w, a, b), x, x)^{+} \\
& \equiv 2((w, a, b), x, x)^{+} \\
& =2\left(\left((w, x, x)^{+}, a, b\right)+\left(w,(x, a, x)^{+}, b\right)+\left(w, a,(x, x, b)^{+}\right)\right) \\
& \equiv 2\left(w,(x, a, x)^{+}, b\right) .
\end{aligned}
$$

Agora, aplicando (4.27), (4.26) e a linearização de (4.46) e pela própria equação (4.46), temos

$$
\begin{aligned}
\left(w, a,(x, x, b)^{+}\right) & =(w \circ a) \circ(x, x, b)^{+}-w \circ\left(a \circ(x, x, b)^{+}\right) \\
& \equiv w \circ\left(a \circ(x, x, b)^{+}\right) \\
& \equiv w \circ\left(x \circ(x, a, b)^{+}\right) \\
& =w \circ\left(x^{2}, a, b\right)^{+} \in \operatorname{Vect}_{\Phi} W .
\end{aligned}
$$


Assim, provamos que $D_{0} \subseteq \operatorname{Vect}_{\Phi} W$.

Provemos agora a inclusão inversa. É claro que $\operatorname{Vect}_{\Phi} W \subseteq D$. Por outro lado, se tomamos $w \in W_{0}$, então $w=(x, y, z) L_{1}^{r_{1}} \cdots L_{7}^{r_{7}}$. Provaremos por indução sobre $n=r_{1}+\cdots+r_{7}$ que $w \in M\left[X_{3}\right]$. Se $n=0$, então pela equação $(4.1), w=(x, y, z)=\frac{1}{6}([x, y, z]+[y, z, x]+[z, x, y]) \in$ $M\left[X_{3}\right]$. Suponhamos que para $n<k$ temos que $w \in M\left[X_{3}\right]$. Consideremos agora $w$ com $r_{1}+\cdots+r_{7}=k$. Podemos supor $r_{7} \neq 0, w=$ $\left((x, y, z) L_{1}^{r_{1}} \cdots L_{7}^{r_{7}-1}, x,[y, z]\right)^{+}$, usando a equação (4.2) e a hipótese de indução, obtemos $w=-\frac{1}{2}(a, b, c)+\frac{1}{4}[b,[a, c]]$, onde $a, b, c \in M\left[X_{3}\right]$. Mas, pela equação (4.1), $w=-\frac{1}{12}([a, b, c]+[b, c, a]+[c, a, b])-\frac{1}{4}[a, c, b] \in$ $M\left[X_{3}\right]$. Agora, se $w \in W_{1}, w=\left[w_{0}, a\right]$, onde $w_{0} \in W_{0}$ e $a \in X_{3}$. Portanto, pelo resultado anterior, $w \in M\left[X_{3}\right]$. Finalmente, se $w \in W_{2}$, então $w=\left(w_{0}, a, b\right)$, onde $w_{0} \in W_{0}$ e $a, b \in X_{3}$. Aplicando novamente a equação (4.1) e em vista do anterior, temos que $w \in M\left[X_{3}\right]$, concluindo assim que $\operatorname{Vect}_{\Phi} W \subseteq M\left[X_{3}\right]$, de onde segue que $\operatorname{Vect}_{\Phi} W=D_{0}$, como queríamos provar.

Lema $26 D=\operatorname{Vect}_{\Phi}\left\{u_{1} R_{u_{2}} \cdots R_{u_{s}} R_{x}^{k_{1}} R_{y}^{k_{2}} R_{z}^{k_{3}} \mid u_{1} \in D_{0}, u_{2}, \ldots, u_{s} \in M\left[X_{3}\right]\right.$, $\left.d\left(u_{i}\right)>1, i=2, \ldots, s: k_{1}, k_{2}, k_{3} \in \mathbb{N} \cup\{0\}\right\}$.

Demonstração: Primeiro provaremos que

$$
A=\operatorname{Vect}_{\Phi}\left\{g\left(a_{1}, \ldots, a_{k}\right) \mid g\left(x_{1}, \ldots, x_{k}\right) \in S J[X], a_{1}, \ldots, a_{k} \in M\left[X_{3}\right]\right\} .
$$

Se chamarmos $T$ ao lado direito da igualdade, temos que $T$ é fechado sob o comutador, ou seja, se $g_{1}, g_{2} \in T$, então $\left[g_{1}, g_{2}\right] \in T$. Com efeito, apliquemos indução sobre $n=d\left(g_{1}\right)+d\left(g_{2}\right)$. Se $n=2$, então $\left[g_{1}, g_{2}\right] \in M\left[X_{3}\right]$ e não há o que provar. Suponhamos a afirmação válida para $d\left(g_{1}\right)+d\left(g_{2}\right)<n$. Se $d\left(g_{1}\right)+$ $d\left(g_{2}\right)=n>2$, então, podemos supor que $d\left(g_{1}\right)>1 \mathrm{e}$, neste caso, escrevamos $g_{1}=g_{1}^{\prime} \circ g_{1}^{\prime \prime} \operatorname{com} g_{1}^{\prime}$ e $g_{1}^{\prime \prime} \in T$. Portanto, usando a linearização parcial de (4.12), temos que $\left[g_{1}, g_{2}\right]=\left[g_{1}^{\prime} \circ g_{1}^{\prime \prime}, g_{2}\right]=g_{1}^{\prime} \circ\left[g_{1}^{\prime \prime}, g_{2}\right]+g_{1}^{\prime \prime} \circ\left[g_{1}^{\prime}, g_{2}\right]$. Mas, pela hipótese de indução, $\left[g_{1}^{\prime \prime}, g_{2}\right],\left[g_{1}^{\prime}, g_{2}\right] \in T$, ficando provada a afirmação.

Voltando ao começo, tomemos $f \in A$ uma palavra não associativa qualquer. Provaremos que $f \in T$, por indução sobre $d(f)$. O resultado é óbvio se $d(f)=1$. Suponhamos que para $d(f)<n, f \in T$. Se $d(f)=n$, então $f=f_{1} f_{2}, \operatorname{com} d(f)_{i}<n, i=1,2$. Mas $f=f_{1} f_{2}=\frac{1}{2}\left[f_{1}, f_{2}\right]+f_{1} \circ f_{2}$. Pela hipótese de indução $f_{1}, f_{2} \in T$, de onde segue, aplicando o anterior, que $f \in T$. A inclusão inversa é imediata. Portanto, $A=T$. 
Se $f=(a, b, c)$, com $a, b, c \in A$, como $A=T$, temos que

$$
f=\sum_{i, j, k} \alpha_{i j k}\left(g_{i}\left(a_{i 1}, \ldots, a_{i l_{i}}\right), g\left(a_{j 1}, \ldots, a_{j l_{j}}\right), g\left(a_{k 1}, \ldots, a_{k l_{k}}\right)\right),
$$

onde $g_{i}, g_{j}, g_{k} \in S J[X]$ e $a_{r s} \in M\left[X_{3}\right], \alpha_{i j k} \in \Phi$. Daqui, usando a equação (4.7), é fácil levar $f$ à seguinte forma:

$$
f=\sum_{i, j, k} \varphi_{i}\left(\left(a_{i}, b_{i}, c_{i}\right), u_{i}, \ldots, v_{i}\right)
$$

onde $\varphi_{i} \in S J[X]$ e $a_{i}, b_{i}, c_{i}, u_{i}, \ldots, v_{i} \in M\left[X_{3}\right]$. Usando novamente a igualdade $a b=\frac{1}{2}[a, b]+a \circ b$ e indução é fácil provar que qualquer elemento do ideal associador $D(A)$ pode ser levado à mesma forma. Considerando a equação $a R_{u \circ v}=-(a, u, v)^{+}+a R_{u} R_{v}$ e transformando $(a, u, v)^{+}$mediante $(4.20) \mathrm{e}$ (4.11), levamos $f$ à seguinte forma:

$$
f=\sum_{i} \alpha_{i}\left(a_{i}, b_{i}, c_{i}\right) R_{u_{i}} \cdots R_{v_{i}}+d_{i}
$$

onde $\alpha_{i} \in F, a_{i}, b_{i}, c_{i}, u_{i}, \ldots, v_{i} \in M\left[X_{3}\right]$ e $d_{i} \in D_{0}$.

Usando agora a equação $a R_{u} R_{v}=a R_{v} R_{u}+(u, a, v)^{+} \mathrm{e}(4.11)$, ordenamos os $R$-operadores obtendo

$$
f=\sum_{j} \beta_{j}\left(a_{j}, b_{j}, c_{j}\right) R_{u_{j}} \cdots R_{v_{j}} R_{x}^{k_{1}} R_{y}^{k_{2}} R_{z}^{k_{3}}+e_{j},
$$

onde $e_{j} \in D_{0}$. Portanto,

$$
\begin{aligned}
& A \subseteq \operatorname{Vect}_{\Phi} \quad\left\{\quad u_{1} R_{u_{2}} \cdots R_{u_{s}} R_{x}^{k_{1}} R_{y}^{k_{2}} R_{z}^{k_{3}} \mid u_{1} \in D_{0},\right. \\
& \left.u_{2}, \ldots, u_{s} \in M\left[X_{3}\right], d\left(u_{i}\right)>1(i=2, \ldots, s), k_{i} \in \mathbb{N} \cup\{0\}\right\} .
\end{aligned}
$$

A inclusão inversa é imediata, ficando provado o lema.

Se chamarmos

$$
\begin{aligned}
& W^{\prime}=\left\{w \circ(x, y, z) \mid w \in W_{0}\right\} \\
& D_{1}=\operatorname{Vect}_{\Phi} W^{\prime} \\
& D_{2}=\operatorname{Vect}_{\Phi}\left\{u_{1} R_{u_{2}} \cdots R_{u_{s}} \mid u_{1} \in D_{0}, u_{2}, \ldots, u_{s} \in M\left[X_{3}\right] \text { e } d\left(u_{i}\right)>1, i=1, \ldots, s\right\}
\end{aligned}
$$

temos o seguinte resultado: 
Lema 27 A seguinte igualdade é satisfeita: $D_{2}=D_{0}+D_{1}$.

Demonstração: A inclusão $D_{0}+D_{1} \subseteq D_{2}$ é imediata do fato que $(x, y, z) \in$ $M\left[X_{3}\right]$. Provemos a inclusão inversa. Seja $u_{1} R_{u_{2}} \cdots R_{u_{s}} \in D_{2}$. Podemos escrever $u_{2}=u_{2}^{\prime}+u_{2}^{\prime \prime}$, onde $u_{2}^{\prime} \in D_{0}$ e $u_{2}^{\prime \prime} \in U=\operatorname{Vect}_{\Phi}\left\{\left(a_{1}, \ldots, a_{n}\right)^{+} \mid a_{i} \in\right.$ $\left.X_{3} \cup\left[X_{3}, X_{3}\right]\right\}$ pois como já vimos $M\left[X_{3}\right] / D_{0}$ é uma álgebra de Lie que pela equação (4.2) está gerada pelos associadores de Jordan em $X_{3} \cup\left[X_{3}, X_{3}\right]$. Pela Proposição 5, podemos supor $u_{1} \in W$. Além disso, por (4.7), a linearização parcial de (4.12) e (4.26), podemos supor $u_{1} \in W_{0}$. daqui e por (4.26) temos que $u_{1} \circ u_{2}^{\prime \prime}=0 \mathrm{e}$, portanto, $u_{1} \circ u_{2}=u_{1} \circ u_{2}^{\prime}$ na decomposição acima.

A seguir, provemos que se $w_{1}, w_{2} \in W_{0}$, então $w_{1} \circ w_{2} \in Z(A)$. Apliquemos indução sobre $n=r_{1}^{(1)}+\cdots+r_{7}^{(1)}+r_{1}^{(2)}+\cdots r_{7}^{(2)}$, onde $w_{i}=$ $(x, y, z) L_{1}^{r_{1}^{(i)}} \cdots L_{7}^{r_{7}^{(i)}}$. Se $n=0$, então o resultado é válido por (4.14). Caso contrário, usando (4.20) e escrevendo $L_{7}=L_{a, b}$, temos que

$w_{1} \circ w_{2}=\left(w_{1} \circ(x, y, z) L_{1}^{r_{1}^{(2)}} \cdots L_{7}^{r_{7}^{(2)}-1}, a, b\right)^{+}-(x, y, z) L_{1}^{r_{1}^{(2)}} \cdots L_{7}^{r_{7}^{(2)}-1} \circ w_{1} L_{7}^{r_{7}^{(2)}}$.

Pela hipótese de indução, o primeiro termo do lado direito é zero e, desta forma, podemos diminuir o grau de $w_{2}$, enquanto aumentamos o grau de $w_{1}$ até chegar a

$$
w_{1} \circ w_{2}= \pm(x, y, z) \circ(x, y, z) L_{1}^{\left(r_{1}^{(1)}+r_{1}^{(2)}\right)} \cdots L_{7}^{\left(r_{7}^{(1)}+r_{7}^{(2)}\right)} .
$$

Agora, usando (4.2) e (4.1), podemos escrever

$$
(x, y, z) L_{1}^{\left(r_{1}^{(1)}+r_{1}^{(2)}\right)} \cdots L_{7}^{\left(r_{7}^{(1)}+r_{7}^{(2)}\right)}=\sum_{i} \alpha_{i}\left[a_{i}, b_{i}\right], \quad a_{i}, b_{i} \in A
$$

de onde segue que $w_{1} \circ w_{2}=\sum \alpha_{i}(x, y, z) \circ\left[a_{i}, b_{i}\right]$, onde $a_{i}, b_{i} \in A$. Daqui e por (4.14), fica provada a afirmação.

Provemos a seguir que se $u_{1} \in W_{0}$ e $u_{2} \in D_{0}$, então $u_{1} \circ u_{2} \in D_{1}$. Separemos nos casos $u_{2} \in W_{i}, i=0,1,2$.

Caso 1: $u_{2} \in W_{0}$. Aplicando indução como na afirmação anterior, vemos que se $n=0$, não há o que provar e, caso contrário, podemos levar $u_{1} \circ u_{2}$ à forma $u_{1} \circ u_{2}=u \circ(x, y, z) \in D_{1}$. 
Caso 2: $u_{2} \in W_{1}$. Podemos supor $u_{2}=[w, x], w \in W_{0}$. Neste caso, usando a linearização parcial de (4.12) e (4.39), temos que

$$
u_{1} \circ u_{2}=u_{1} \circ[w, x]=\left[w, u_{1} \circ x\right]-\left[w, u_{1}\right] \circ x=\left[w, u_{1} \circ x\right] .
$$

Aplicando (4.27), (4.33) e novamente (4.12) e (4.39), podemos passar os $L$ operadores de um lado para o outro do comutador, obtendo

$$
\begin{aligned}
{\left[w, u_{1} \circ x\right] } & =\left[u_{1}, w \circ x\right] \\
& =\left[u_{1}, w\right] \circ x+\left[u_{1}, x\right] \circ w \\
& =w \circ\left[u_{1}, x\right] .
\end{aligned}
$$

Mas, por outro lado,

$$
u_{1} \circ u_{2}=u_{1} \circ[w, x]=\left[u_{1} \circ w, x\right]-w \circ\left[u_{1}, x\right]=-w \circ\left[u_{1}, x\right] .
$$

Portanto, $u_{1} \circ u_{2}=0 \in D_{1}$.

O terceiro caso é análogo ao segundo, usando (4.34) e (4.40) em vez de (4.33) e (4.39) e (4.7) no lugar de (4.12), obtendo-se que $u_{1} \circ(w, a, b)=0$ se $w \in W_{0}$ e $a, b \in X_{3}$. Desta forma, provamos que $W_{0} \circ D_{0} \subseteq D_{1}$ e, assim, na expressão original $u_{1} R_{u_{2}}=u_{1} R_{u_{2}^{\prime}}=u_{2} \circ u_{2}^{\prime} \in D_{1}$.

Daqui, considerando a decomposição dos elementos de $M\left[X_{3}\right]$, e por un argumento de indução sobre $s$, para provar o lema é suficiente demonstrar que se $v \in D_{1}$ e $u_{3} \in M\left[X_{3}\right]$, com $d\left(u_{3}\right)>1$, então $v \circ u_{3} \in D_{1}$. Consideremos $u_{1} \in W_{0}, u_{3} \in M\left[X_{3}\right]$ e $d\left(u_{3}\right)>1$. Como já vimos, $W_{0} \circ W_{1}=0, W_{0} \circ W_{2}=0$ e de (4.26) segue que $W_{0} \circ U=0$. Portanto, se $u_{3} \in W_{1} \cup W_{2} \cup U$, temos que $\left(u_{1} \circ(x, y, z)\right) \circ u_{3}=\left((x, y, z), u_{1}, u_{3}\right)^{+}$. Mas pelas equações (4.1) $\mathrm{e}$ (4.2) se desprende que $W_{1} \cup W_{2} \cup U \in M\left[X_{3}\right]$, além do mais é claro que $\left((x, y, z), u_{1}, u_{3}\right)^{+} \in D$ e portanto, neste caso $\left((x, y, z), u_{1}, u_{3}\right)^{+} \in D_{0}$. Se $u_{3} \in W_{0}$, escrevendo $u_{3}=(x, y, z) L_{1}^{r_{1}} \cdots L_{7}^{r_{7}}$ e usando (4.41), temos que

$$
\begin{aligned}
\left(u_{1} \circ(x, y, z)\right) \circ u_{3} & =\left((x, y, z), u_{1}, u_{3}\right)^{+}+(x, y, z) \circ\left(u_{1} \circ u_{3}\right) \\
& =(x, y, z) \circ\left(u_{1} \circ u_{3}\right) .
\end{aligned}
$$

Usando (4.20) várias vezes, (4.25) e o fato de que $W_{0} \circ W_{0} \in Z(A)$, obtemos

$$
u_{1} \circ u_{3}=u_{1} L_{1}^{r_{1}} \cdots L_{7}^{r_{7}} \circ(x, y, z)=u_{1}^{\prime} \circ(x, y, z) .
$$

Portanto,

$(x, y, z) \circ\left(u_{1} \circ u_{3}\right)=(x, y, z) \circ\left(u_{1}^{\prime} \circ(x, y, z)\right)=\left(u_{1}^{\prime},(x, y, z),(x, y, z)\right)^{+}+u_{1}^{\prime} \circ(x, y, z)^{2}$, 
o primeiro termo desta soma sendo zero por causa de (4.41). Por outro lado, usando (4.2), (4.26), a linearização parcial de (4.12) e (4.16), temos

$$
\begin{aligned}
(x, y, z)^{2} & =(x, y, z) \circ\left(-\frac{1}{2}[x,[y, z]]-\frac{1}{4}(x, y, z)^{+}\right) \\
& =-\frac{1}{2}(x, y, z) \circ[x,[y, z]] \\
& =\frac{1}{2}[x,(x, y, z)] \circ[y, z]-\frac{1}{2}[x,(x, y, z) \circ[y, z]] \\
& =\frac{1}{2}[x,(x, y, z)] \circ[y, z] .
\end{aligned}
$$

Portanto, aplicando (4.26) obtemos

$$
\left(u_{1} \circ(x, y, z)\right) \circ u_{3}=-\left(u_{1}^{\prime},[x, z],[x,(x, y, z)]\right)^{+}
$$

Usando novamente (4.1) e (4.2) vemos que $-\left(u_{1}^{\prime},[x, z],[x,(x, y, z)]\right)^{+} \in D_{0}$. Desta forma, aplicando indução sobre $s$ no elemento $u_{1} R_{u_{2}} \cdots R_{u_{s}}$ o lema fica provado.

Considerando $B=\left\{w R_{x}^{k_{1}} R_{y}^{k_{2}} R_{z}^{k_{3}} \mid k_{i} \in \mathbb{N} \cup\{0\}, i=1,2,3, w \in W \cup W^{\prime}\right\}$, temos o seguinte resultado.

Teorema 8 (Il'tyakov) $T(C) \cap \operatorname{Vect}_{\Phi} B=(0)$.

Demonstração: Consideremos $f \in T(C) \cap \operatorname{Vect}_{\Phi} B$. Podemos escrever

$$
f=\sum_{k_{1}, k_{2}, k_{3}} f_{k_{1}, k_{2}, k_{3}} R_{x}^{k_{1}} R_{y}^{k_{2}} R_{z}^{k_{3}}
$$

onde $f_{k_{1}, k_{2}, k_{3}} \in \operatorname{Vect}_{\Phi}\left(W \cup W^{\prime}\right)$ e $f=0$ é uma identidade na álgebra $C$ (álgebra de Cayley-Dickson). Aplicando os operadores $\Delta_{a}, a \in X_{3}$, é fácil provar (ver (12, p.19)) que $f_{k_{1}, k_{2}, k_{3}} \in \operatorname{Vect}_{\Phi}\left(W \cup W^{\prime}\right)$ pelo que podemos supor $f \in \operatorname{Vect}_{\Phi}\left(W \cup W^{\prime}\right)$. Escrevamos $f=f^{\prime}+f^{\prime \prime}$, onde $f^{\prime \prime} \in \operatorname{Vect}_{\Phi} W^{\prime}$ e $f^{\prime} \in \operatorname{Vect}_{\Phi} W$. Agora, usando as equações (4.2) e (4.1), podemos escrever $f^{\prime}=\sum_{i}\left[a_{i}, b_{i}\right], a_{i}, b_{i} \in A_{3}$ e daí, como o traço de um comutador é zero, temos que se $C_{7}^{(-)}$é o conjunto de elementos de traço zero, então $f^{\prime}: C \rightarrow$ $C_{7}^{(-)}$. Além disso, pela equação $(4.1), \operatorname{tr}((x, y, z))=0$, de onde segue que $(x, y, z)^{2} \in F$ e $f^{\prime \prime}: C \rightarrow F$. Portanto, como $f^{\prime}+f^{\prime \prime}=0$, temos que $f^{\prime}=0$ e $f^{\prime \prime}=0$ são identidades de $C$. 
Consideremos $f^{\prime \prime}=\left(\sum_{r_{1}, \ldots, r_{7}} \alpha_{r_{1}, \ldots, r_{7}}(x, y, z) L_{1}^{r_{1}} \cdots L_{7}^{r_{7}}\right) \circ(x, y, z)$ sobre $C_{7}^{(-)}$. Observemos que se $a \in C_{7}^{(-)}$, então $a^{2} \in \Phi$ e daí $\left[a^{2}, b\right]=0$, para todo $b \in C$. Desta identidade fraca segue que todos os elementos da forma $a \circ c \in K(C)$. Além do mais, como $C$ é uma álgebra alternativa e $3 \in \Phi$, temos que $K(C) \subset N(C)$ (ver [12, p.136]). Portanto, os elementos da forma $a \circ c$ pertencem ao centro de $C$. Observemos que se $L=L_{a, b}$, então pelas equações (4.9) e (4.24), $(x, y, z) L_{a, b}=(x, y, z) L_{b, a}$. Portanto, podemos supor $d(b)=1$. Além disso, como os elementos da forma $c \circ d \in Z(C)$, podemos fazer a seguinte tranformação:

$$
\begin{aligned}
((x, y, z), a, b)^{+} \circ(x, y, z)= & ((x, y, z),(x, y, z) \circ a, b)^{+} \\
& -((x, y, z),(x, y, z), b)^{+} \circ a \\
= & ((x, y, z),(x, y, z), b)^{+} \circ a \\
= & \left.\left((x, y, z)^{2} \circ b\right) \circ a-((x, y, z) \circ(x, y, z) \circ b)\right) \circ a \\
= & \left((x, y, z)^{2}, b, a\right)^{+}+(x, y, z)^{2} \circ(b \circ a) \\
& -((x, y, z) \circ((x, y, z) \circ b)) \circ a \\
= & (x, y, z)^{2} \circ(b \circ a)-((x, y, z) \circ((x, y, z) \circ b)) \circ a .
\end{aligned}
$$

Podemos supor $b=x$ e daí pela equação (1.3), temos que $(x, y, z) \circ b=(x, y \circ$ $x, z)=0$ e portanto $(x, y, z) L_{a, b} \circ(x, y, z)=(x, y, z)^{2} \circ(a \circ b)$. Generalizando este processo a mais operadores podemos escrever

$$
f^{\prime \prime}=(x, y, z)^{2} \circ \sum \alpha_{r_{1} \cdots r_{7}}(x \circ y)^{r_{1}} \cdots(x \circ[y, z])^{r_{7}} .
$$

Usando (4.7) e o lema 24 podemos expressar $(x, y, z)^{2}$ como um polinômio associativo e comutativo $\varphi$ em $(x \circ y), \ldots,(x \circ[y, z])$, obtendo desta forma $f^{\prime \prime}=\sum \gamma_{r_{1}^{\prime} \cdots r_{7}^{\prime}}(x \circ y)^{r_{1}^{\prime}} \ldots(x \circ[y, z])^{r_{7}^{\prime}}$.

$\mathrm{O}$ conjunto de 7 -uplas $\left(\beta_{1}, \ldots, \beta_{7}\right) \in \Phi \times \cdots \times \Phi$ tais que existe $x, y, z \in$ $C_{7}^{(-)}$, tais que $x \circ y=\beta_{1}, \cdots, x \circ[y, z]=\beta_{7}$ e $\varphi\left(\beta_{1}, \ldots, \beta_{7}\right) \neq 0$ é aberto na topologia de Zariski (ver $\left[11\right.$, p.90]). Como $\Phi$ é infinito, temos que $\gamma_{r_{1}^{\prime} \cdots r_{7}^{\prime}}=0$ e, portanto, $f^{\prime \prime}=0$.

Por outro lado, podemos escrever

$$
f^{\prime}=w_{1}+\left[w_{2}, x\right]+\left[w_{3}, y\right]+\cdots+\left(w_{7}, z, x\right),
$$

onde $w_{i}=\sum \alpha_{r_{1}^{(i)} \ldots r_{7}^{(i)}}(x, y, z) L_{1}^{r_{1}^{(i)}} \cdots L_{7}^{r_{7}^{(i)}}$. Como no caso anterior, o valor de $f^{\prime}$ em $C_{7}^{(-)}$é dado por

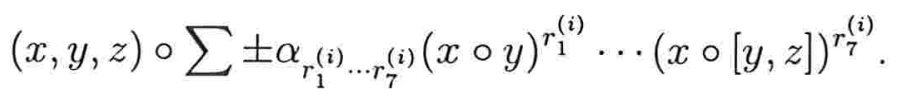


Colocando $u_{1}=(x, y, z), u_{2}=[(x, y, z), x], \ldots, u_{7}=((x, y, z), z, x)$ e $u_{1}^{\prime}=$ $(x, y, z), u_{2}^{\prime}=-\frac{1}{2}[y, z], \ldots, u_{7}^{\prime}=-y$, temos que $u_{i} \circ u_{j}^{\prime}=\delta_{i j}(x, y, z)^{2}$. Com efeito, por simetria, é suficiente testar uns poucos casos:

$$
\begin{aligned}
u_{1} \circ u_{1}^{\prime} & =(x, y, z)^{2}, \\
u_{1} \circ u_{2}^{\prime} & =(x, y, z) \circ[y, z]=0 \text { (pela eq. 13), } \\
u_{1} \circ u_{7}^{\prime} & =(x, y, z) \circ(-y)=-(x, y, y \circ z)=0, \\
u_{2} \circ u_{1}^{\prime} & =[(x, y, z), x] \circ(x, y, z) \\
& =[(x, y, z), x \circ(x, y, z)]-[(x, y, z),(x, y, z)] \circ x=0, \\
u_{2} \circ u_{2}^{\prime} & =[(x, y, z), x] \circ-\frac{1}{2}[y, z] \\
& \left.=2(x, y, z) \circ(x, y, z)^{+}+(x, y, z) \circ(x, y, z)=(x, y, z)^{2} \quad \text { pela eq. } 26\right), \\
u_{2} \circ u_{7}^{\prime} & =[(x, y, z), x] \circ y=[(x, y, z) \circ y, x]-(x, y, z) \circ[y, x]=0, \\
u_{7} \circ u_{1}^{\prime} & =((x, y, z), z, x) \circ(x, y, z) \\
& =((x, y, z), z \circ(x, y, z), x)-((x, y, z),(x, y, z) x) \circ z=0, \\
u_{7} \circ u_{2}^{\prime} & =((x, y, z), z, x) \circ-\frac{1}{2}[y, z] \\
& =-\frac{1}{2}((x, y, z) \circ[y, z], z, x)+\frac{1}{2}([y, z], z, x) \circ(x, y, z) \\
& =\frac{1}{2}[(x, y, z), z] \circ(x, y, z)=0, \\
u_{7} \circ u_{7}^{\prime} & =((x, y, z), z, x) \circ(-y) \\
& =-((x, y, z) \circ y, z, x)+(y, z, x) \circ(x, y, z)=(x, y, z)^{2},
\end{aligned}
$$

para quaisquer $x, y, z \in C_{7}^{(-)}$. Desta forma,

$$
f^{\prime} \circ u_{i}^{\prime}=(x, y, z)^{2} \circ \sum \alpha_{r_{1}^{(i)} \ldots r_{7}^{(i)}}(x \circ y)^{r_{1}^{(i)} \ldots r_{7}^{(i)}}
$$

e como antes, $\alpha_{r_{1}^{(i)} \ldots r_{7}^{(i)}}=0$, ficando provado que $f^{\prime}=0$ e o Teorema 8 .

Notemos que a independência linear de $B$ segue da demonstração de que $f^{\prime}=0$ e portanto $B$ é uma base de $D\left(A_{3}\right)$ concluindo assim que $T(C) \cap$ $D\left(A_{3}\right)=(0)$. Observemos também que como $A_{3} / D\left(A_{3}\right) \cong A s s\left[X_{3}\right]$ é fácil encontrar uma base de $A_{3}$ a partir de $B$. 


\section{Referências Bibliográficas}

[1] G.V. Dorofeev: An example of an solvable but not nilpotent alternative ring, Usp. Mat. Nauka, 15 (3): 147-150 (1960).

[2] G.V. Dorofeev: Centers of nonassociative rings, Algebra i Logika, 12 (5): 530-549 (1973).

[3] G. V. Dorofeev: An example in the theory of alternative rings, sib. Math. $Z h, 4$ (5): 1049-1052 (1963).

[4] E. Kleinfeld e M. Humm: On free alternative rings, J. Comb. Theory ,2: 140-144 (1967).

[5] A. V. Il'tyakov: Free alternative algebras of rank 3, Algebra i Logika, 23 (2): 136-158 (1984).

[6] N. Jacobson : Structure and representation of Jordan algebras. American Mathematical Society: (1968).

[7] Yu. P. Razmyslov: On existence of a finite basis for the identidies of the matrix algebra of second order over a field of characteristic zero, Algebra i Logica, 12 (1): 83-113 (1973).

[8] A. Sagle: Malt'sev algebras, Trans. Am. Math. Soc. 101 (3): 426-458 (1961).

[9] I. P. Shestakov: Centers of alternative algebras, Algebra i Logika, 15(3): 343-362 (1976).

[10] I. P. Shestakov: On a problem of Shirshov, Algebra i Logika, 16 (2): 153-166 (1978).

[11] Stephen Willard: General topology, Addison-Wesley: (1978). 
[12] K. A. Zhevlakov, A. M. Slin'ko, I. P. Shestakov, A. I. Shirshov: Rings that are nearly associative, Nauka, Moscow: (1978). 